\title{
Discrete flavour symmetries, neutrino mixing and leptonic CP violation
}

\author{
S. T. Petcov ${ }^{1,2, a}$ \\ ${ }^{1}$ SISSA/INFN, Via Bonomea 265, 34136 Trieste, Italy \\ ${ }^{2}$ Kavli IPMU (WPI), University of Tokyo, 5-1-5 Kashiwanoha, Kashiwa 277-8583, Japan
}

Received: 30 July 2018 / Accepted: 15 August 2018 / Published online: 4 September 2018

(C) The Author(s) 2018

\begin{abstract}
The current status of our knowledge of the 3neutrino mixing parameters and of the $\mathrm{CP}$ violation in the lepton sector is summarised. The non-Abelian discrete symmetry approach to understanding the observed pattern of neutrino mixing and the related predictions for neutrino mixing angles and leptonic Dirac CP violation are reviewed. Possible tests of these predictions using the existing data on neutrino mixing angles as well as prospective data from current and future neutrino oscillation experiments (T2K, NOvA, Daya Bay, T2HK, T2HKK, DUNE) are also discussed.
\end{abstract}

\section{Introduction}

Understanding the origins of the patterns of neutrino mixing and of neutrino mass squared differences, revealed by the data obtained in the neutrino oscillation experiments (see, e.g., [1]), is one of the most challenging problems in neutrino physics. It is part of the more general fundamental problem in particle physics of understanding the origins of flavour, i.e., of the patterns of quark, charged lepton and neutrino masses, and of the quark and lepton mixing.

We believe, and we are not alone in holding this view, that with the observed pattern of neutrino mixing Nature is "sending" us a Message. The Message is encoded in the values of the neutrino mixing angles, leptonic $\mathrm{CP}$ violation (CPV) phases in the Pontecorvo, Maki, Nakagawa and Sakata (PMNS) neutrino mixing matrix [2-5] and neutrino masses. We do not know at present what is the content of Nature's Message. However, on the basis of the current ideas about the possible origins of the observed pattern of neutrino mixing, the Nature's Message can have two completely different contents, each of which can be characterised by one word: ANARCHY or SYMMETRY. In the ANARCHY approach [6-8] to understanding the pattern of neutrino mix-

\footnotetext{
a e-mail: petcov@sissa.it
}

ing it is assumed that Nature "threw dice" when "choosing" the values of the neutrino masses, mixing angles and leptonic CPV phases. The main prediction of the ANARCHY explanation of the pattern of neutrino mixing is the absence of whatever correlations between the values of the neutrino masses, between the values of the neutrino mixing angles, and between the values of the neutrino mixing angles and the CPV phases, all of them being random quantities. As a consequence, no specific values of, e.g., neutrino mixing angles are predicted: the predictions of these (and other leptonic) measurable quantities are in the form of distributions. In contrast, one of the main characteristic features of the SYMMETRY approach to neutrino mixing is the prediction of the values of some of the mixing angles and/or of the existence of correlations between the values of at least some of the observables (angles, CPV phases) of the the neutrino mixing matrix.

Within the SYMMETRY approach, the observed pattern of neutrino mixing, which differs drastically from the quark mixing pattern, can be naturally understood on the basis of specific class of symmetries - the class of non-Abelian discrete flavour symmetries (see, e.g., [9-11]). Thus, the specific form of the neutrino mixing can have its origin in the existence of new fundamental symmetry in the lepton sector. The most distinctive feature of the approach to neutrino mixing based on non-Abelian discrete flavour symmetries is the predictions of the values of some of the neutrino mixing angles and leptonic CPV phases, and/or of existence of correlations between the values of at least some the neutrino mixing angles and/or between the values of the neutrino mixing angles and the Dirac CPV phase in the PMNS matrix, etc. (see, e.g., [11-20]). Combining the discrete symmetry approach with the idea of generalised $\mathrm{CP}$ invariance [21-23] - a generalisation of the standard CP invariance requirement - allows to obtain predictions also for the Majorana CPV phases in the PMNS matrix in the case of massive Majorana neutrinos (see, e.g., [24-36] and references quoted 
therein). Most importantly, these predictions and predicted correlations, and thus the discrete symmetry approach itself, can be tested experimentally (see, e.g., [12,13,25,37-42]).

In the present article we review aspects of the symmetry approach to neutrino mixing based on the class of non-Abelian discrete flavour symmetries, which is widely explored at present (see, e.g., $[11,35,36,43,44]$ and references quoted therein. ${ }^{1}$ ) We will discuss also the typical phenomenological predictions of the approach and their possible tests in currently running and future planned neutrino oscillation experiments.

Before discussing the discrete flavour symmetry approach to neutrino mixing we would like to review briefly the current status of our knowledge of neutrino masses, neutrino mixing and leptonic CPV phases, the remaining fundamental problems in neutrino physics and the future prospects in this field.

\section{The three-neutrino mixing}

The experiments with solar, atmospheric, reactor and accelerator neutrinos have provided compelling evidences for the existence of neutrino oscillations [2-4] - transitions in flight between the different flavour neutrinos $v_{e}, v_{\mu}, v_{\tau}$ (antineutrinos $\bar{v}_{e}, \bar{v}_{\mu}, \bar{v}_{\tau}$ ) - caused by nonzero neutrino masses and neutrino mixing (see, e.g., Ref. [1] for review of the relevant data). The existence of flavour neutrino oscillations implies the presence of mixing in the weak charged lepton current:

$$
\begin{aligned}
\mathcal{L}_{\mathrm{CC}} & =-\frac{g}{\sqrt{2}} \sum_{l=e, \mu, \tau} \overline{l_{L}}(x) \gamma_{\alpha} \nu_{l \mathrm{~L}}(x) W^{\alpha \dagger}(x)+\text { h.c. }, \\
\nu_{l \mathrm{~L}}(x) & =\sum_{j=1}^{n} U_{l j} v_{j \mathrm{~L}}(x),
\end{aligned}
$$

where $v_{l L}(x)$ are the flavour neutrino fields, $v_{j \mathrm{~L}}(x)$ is the lefthanded (LH) component of the field of the neutrino $v_{j}$ having a mass $m_{j}$, and $U$ is a unitary matrix - the PMNS neutrino mixing matrix [2-5]. All compelling neutrino oscillation data can be described assuming 3-neutrino mixing in vacuum, $n=3$. The number of massive neutrinos $n$ can, in general, be bigger than 3 if, e.g., there exist RH sterile neutrinos [5] and they mix with the LH flavour neutrinos. It follows from the current data that at least 3 of the neutrinos $v_{j}$, say $v_{1}$, $v_{2}, v_{3}$, must be light, i.e., must have masses smaller than roughly $1 \mathrm{eV}, m_{1,2,3} \lesssim 1 \mathrm{eV}$, and must have different masses, $m_{1} \neq m_{2} \neq m_{3}{ }^{2}$

\footnotetext{
${ }^{1}$ For early attempts see, e.g., [45-47].

2 At present there are several experimental inconclusive hints for existence of one or two light sterile neutrinos at the $\mathrm{eV}$ scale, which mix with the flavour neutrinos, implying the presence in the neutrino mixing of additional one or two neutrinos, $v_{4}$ or $v_{4,5}$, with masses $m_{4}\left(m_{4,5}\right) \sim 1 \mathrm{eV}$ (see, e.g., Ref. [48]). For a recent discussion of these hints and of the related implications see, e.g., Refs. [49,50].
}

In the case of 3 light neutrinos, the $3 \times 3$ unitary neutrino mixing matrix $U$ can be parametrised, as is well known, by 3 angles and, depending on whether the massive neutrinos $v_{j}$ are Dirac or Majorana particles, by one Dirac, or one Dirac and two Majorana, $\mathrm{CP}$ violation (CPV) phases [51]:

$U=V P, \quad P=\operatorname{diag}\left(1, e^{i \frac{\alpha_{21}}{2}}, e^{i \frac{\alpha_{31}}{2}}\right)$,

where $\alpha_{21,31}$ are the two Majorana CPV phases and in the "standard" parametrisation the matrix $V$ is given by:

$V=\left(\begin{array}{ccc}c_{12} c_{13} & s_{12} c_{13} & s_{13} e^{-i \delta} \\ -s_{12} c_{23}-c_{12} s_{23} s_{13} e^{i \delta} & c_{12} c_{23}-s_{12} s_{23} s_{13} e^{i \delta} & s_{23} c_{13} \\ s_{12} s_{23}-c_{12} c_{23} s_{13} e^{i \delta} & -c_{12} s_{23}-s_{12} c_{23} s_{13} e^{i \delta} & c_{23} c_{13}\end{array}\right)$.

In Eq. (3), $c_{i j}=\cos \theta_{i j}, s_{i j}=\sin \theta_{i j}$, the angles $\theta_{i j} \in$ $[0, \pi / 2]$, and $\delta \in[0,2 \pi)$ is the Dirac CPV phase. Thus, in the case of massive Dirac neutrinos, the neutrino mixing matrix $U$ is similar, in what concerns the number of mixing angles and CPV phases, to the Cabibbo, Kobayashi, Maskawa (CKM) quark mixing matrix. The PMNS matrix $U$ contains two additional physical CPV phases if $v_{j}$ are Majorana particles due to the special properties of Majorana fermions (see, e.g., Refs. [51-53]). On the basis of the existing neutrino data it is impossible to determine whether the massive neutrinos are Dirac or Majorana fermions.

The probabilities of neutrino oscillations are functions of the neutrino energy, $E$, the source-detector distance $L$, of the elements of $U$ and, for relativistic neutrinos used in all neutrino experiments performed so far, of the neutrino mass squared differences $\Delta m_{i j}^{2} \equiv\left(m_{i}^{2}-m_{j}^{2}\right), i \neq j$ (see, e.g., Ref. [52]). In the case of 3-neutrino mixing there are only two independent $\Delta m_{i j}^{2}$, say $\Delta m_{21}^{2} \neq 0$ and $\Delta m_{31}^{2} \neq 0$. The numbering of neutrinos $v_{j}$ is arbitrary. We will employ the widely used convention which allows to associate $\theta_{13}$ with the smallest mixing angle in the PMNS matrix, and $\theta_{12}, \Delta m_{21}^{2}>0$, and $\theta_{23}, \Delta m_{31}^{2}$, with the parameters which drive the solar $\left(v_{e}\right)$ and the dominant atmospheric $v_{\mu}$ and $\bar{v}_{\mu}$ oscillations, respectively. In this convention $m_{1}<m_{2}$, $0<\Delta m_{21}^{2}<\left|\Delta m_{31}^{2}\right|$, and, depending on $\operatorname{sgn}\left(\Delta m_{31}^{2}\right)$, we have either $m_{3}<m_{1}$ or $m_{3}>m_{2}$.

The existing data, accumulated over many years of studies of neutrino oscillations, allow us to determine $\Delta m_{21}^{2}, \theta_{12}$, and $\left|\Delta m_{31(32)}^{2}\right|, \theta_{23}$ and $\theta_{13}$, with a relatively high precision $[54,55]$. Since 2013 there are also persistent hints that the Dirac CPV phase $\delta$ has a value close to $3 \pi / 2$ (see [56]). The best fit values (b.f.v.) and the $2 \sigma$ and $3 \sigma$ allowed ranges of $\Delta m_{21}^{2}, s_{12}^{2},\left|\Delta m_{31(32)}^{2}\right|, s_{23}^{2}, s_{13}^{2}$ and $\delta$, found in the analysis of global neutrino oscillation data performed in [54] are given in Table 1. Similar results were obtained in Ref. [55].

In both analyses $[54,55]$ the authors find, in particular, that the best fit value of the Dirac CPV phases $\delta$ is close to $3 \pi / 2$ : 
Table 1 The best fit values, $2 \sigma$ and $3 \sigma$ ranges of the neutrino oscillation parameters obtained in the global analysis of the neutrino oscillation data performed in [54]. (The Table is taken from Ref. [35].)

\begin{tabular}{llll}
\hline Parameter & Best fit value & $2 \sigma$ range & $3 \sigma$ range \\
\hline $\sin ^{2} \theta_{12} / 10^{-1}$ & 2.97 & $2.65-3.34$ & $2.50-3.54$ \\
$\sin ^{2} \theta_{13} / 10^{-2}(\mathrm{NO})$ & 2.15 & $1.99-2.31$ & $1.90-2.40$ \\
$\sin ^{2} \theta_{13} / 10^{-2}(\mathrm{IO})$ & 2.16 & $1.98-2.33$ & $1.90-2.42$ \\
$\sin ^{2} \theta_{23} / 10^{-1}(\mathrm{NO})$ & 4.25 & $3.95-4.70$ & $3.81-6.15$ \\
$\sin ^{2} \theta_{23} / 10^{-1}(\mathrm{IO})$ & 5.89 & $3.99-4.83 \oplus 5.33-6.21$ & $3.84-6.36$ \\
$\delta / \pi(\mathrm{NO})$ & 1.38 & $1.00-1.90$ & $0-0.17 \oplus 0.76-2$ \\
$\delta / \pi(\mathrm{IO})$ & 1.31 & $0.92-1.88$ & $0-0.15 \oplus 0.69-2$ \\
$\Delta m_{21}^{2} / 10^{-5} \mathrm{eV}^{2}$ & 7.37 & $7.07-7.73$ & $6.93-7.96$ \\
$\Delta m_{31}^{2} / 10^{-3} \mathrm{eV}^{2}(\mathrm{NO})$ & 2.56 & $2.49-2.64$ & $2.45-2.69$ \\
$\Delta m_{23}^{2} / 10^{-3} \mathrm{eV}^{2}(\mathrm{IO})$ & 2.54 & $2.47-2.62$ & $2.42-2.66$ \\
\hline
\end{tabular}

in [54], for example, the authors find $\delta=1.38 \pi$ (1.31 $\pi$ ) for $\Delta m_{31(32)}^{2}>0\left(\Delta m_{31(32)}^{2}<0\right)$. The absolute $\chi^{2}$ minimum takes place for $\Delta m_{31(32)}^{2}>0$. According to Ref. [54], the $\mathrm{CP}$ conserving values $\delta=0$ or $2 \pi$ are disfavored at $2.4 \sigma$ $(3.2 \sigma)$ for $\Delta m_{31(32)}^{2}>0\left(\Delta m_{31(32)}^{2}<0\right)$; the $\mathrm{CP}$ conserving value $\delta=\pi$ in the case of $\Delta m_{31(32)}^{2}>0\left(\Delta m_{31(32)}^{2}<0\right)$ is statistically approximately $2.0 \sigma(2.5 \sigma)$ away from the best fit value $\delta \cong 1.38 \pi(1.31 \pi)$. In what concerns the $\mathrm{CP}$ violating value $\delta=\pi / 2$, it is strongly disfavored at $3.4 \sigma(3.9 \sigma)$ for $\Delta m_{31(32)}^{2}>0\left(\Delta m_{31(32)}^{2}<0\right){ }^{3}$ At $3 \sigma, \delta / \pi$ is found to lie in the case of $\Delta m_{31(32)}^{2}>0\left(\Delta m_{31(32)}^{2}<0\right)$ in the following intervals [54]: $(0.00-0.17(0.15)) \oplus(0.76(0.69)-2.00))$. The results on $\delta$ obtained in [55] differ somewhat from, but are compatible at $1 \sigma$ confidence level (C.L.) with, those found in [54].

It follows also from the results quoted in Table 1 that $\Delta m_{21}^{2} /\left|\Delta m_{31(32)}^{2}\right| \cong 0.03$. We have $\left|\Delta m_{31}^{2}\right|=\mid \Delta m_{32}^{2}-$ $\Delta m_{21}^{2}|\cong| \Delta m_{32}^{2} \mid$. The angle $\theta_{12}$ is definitely smaller than $\pi / 4$ : the value of $\theta_{12}=\pi / 4$, i.e., maximal solar neutrino mixing, is ruled out at high confidence level (C.L.) by the data - one has $\cos 2 \theta_{12} \geq 0.29$ at $99.73 \%$ C.L. The quoted results imply also that the value of $\theta_{23}$ can deviate by approximately \pm 0.1 from $\pi / 4, \theta_{12} \cong \pi / 5.4$ and that $\theta_{13} \cong \pi / 20$. Thus, the pattern of neutrino mixing differs drastically from the pattern of quark mixing.

It should be noted that in the more recent global analyses $[57,58]$, which used, in particular, updated results on $\sin ^{2} \theta_{23}$ from the NOvA experiment, the best fit value of $\sin ^{2} \theta_{23}$ for NO spectrum was found to be larger than $0.5^{4}$ :

\footnotetext{
3 The quoted confidence levels for $\delta=0, \pi$ and $\pi / 2$ are all with respect to the absolute $\chi^{2}$ minimum.

${ }^{4}$ In what concerns the other two neutrino mixing angles $\theta_{12}$ and $\theta_{13}$, the results reported in $[54,57,58]$ differ insignificantly.
}

$$
\begin{aligned}
& \sin ^{2} \theta_{23}=0.538(0.554)[57], \\
& \sin ^{2} \theta_{23}=0.551(0.557)[58], \quad \text { NO (IO). }
\end{aligned}
$$

Apart from the hint that the Dirac phase $\delta \sim 3 \pi / 2$, no other experimental information on the Dirac and Majorana CPV phases in the neutrino mixing matrix is available at present. Thus, the status of CP symmetry in the lepton sector is essentially unknown. With $\theta_{13} \cong 0.15 \neq 0$, the Dirac phase $\delta$ can generate $\mathrm{CP}$ violating effects in neutrino oscillations $[51,59,60]$, i.e, a difference between the probabilities of the $v_{l} \rightarrow v_{l^{\prime}}$ and $\bar{v}_{l} \rightarrow \bar{v}_{l^{\prime}}$ oscillations, $l \neq l^{\prime}=e, \mu, \tau$. The magnitude of CP violation in $\nu_{l} \rightarrow \nu_{l^{\prime}}$ and $\bar{v}_{l} \rightarrow \bar{v}_{l^{\prime}}$ oscillations in vacuum, $l \neq l^{\prime}=e, \mu, \tau$, is determined by [61] the rephasing invariant $J_{C P}$, associated with the Dirac CPV phase in $U$ :

$J_{\mathrm{CP}}=\operatorname{Im}\left(U_{\mu 3} U_{e 3}^{*} U_{e 2} U^{*}{ }_{\mu 2}\right)$

It is analogous to the rephasing invariant associated with the Dirac CPV phase in the CKM quark mixing matrix [62]. In the standard parametrisation of the neutrino mixing matrix (3), $J_{\mathrm{CP}}$ has the form:

$$
\begin{aligned}
J_{\mathrm{CP}} & \equiv \operatorname{Im}\left(U_{\mu 3} U_{e 3}^{*} U_{e 2} U_{\mu 2}^{*}\right) \\
& =\frac{1}{8} \cos \theta_{13} \sin 2 \theta_{12} \sin 2 \theta_{23} \sin 2 \theta_{13} \sin \delta .
\end{aligned}
$$

Thus, given the fact that $\sin 2 \theta_{12}, \sin 2 \theta_{23}$ and $\sin 2 \theta_{13}$ have been determined experimentally with a relatively good precision, the size of $\mathrm{CP}$ violation effects in neutrino oscillations depends essentially only on the magnitude of the currently not well determined value of the Dirac phase $\delta$. The current data imply $0.026(0.027)|\sin \delta| \lesssim\left|J_{\mathrm{CP}}\right| \lesssim 0.035|\sin \delta|$, where we have used the $3 \sigma$ ranges of $\sin ^{2} \theta_{12}, \sin ^{2} \theta_{23}$ and $\sin ^{2} \theta_{13}$ given in Table 1 . For the current best fit values of $\sin ^{2} \theta_{12}, \sin ^{2} \theta_{23}, \sin ^{2} \theta_{13}$ and $\delta$ we find in the case of $\Delta m_{31(2)}^{2}>0\left(\Delta m_{31(2)}^{2}<0\right): J_{\mathrm{CP}} \cong 0.032 \sin \delta \cong-0.030$ $\left(J_{\mathrm{CP}} \cong 0.032 \sin \delta \cong-0.027\right)$. Thus, if the indication that $\delta$ has a value close to $3 \pi / 2$ is confirmed by future more precise 
data, (i) the $J_{\mathrm{CP}}$ factor in the lepton sector would be approximately by 3 orders of magnitude larger in absolute value than corresponding $J_{\mathrm{CP}}$ factor in the quark sector, and (ii) the $\mathrm{CP}$ violation effects in neutrino oscillations would be relatively large and observable.

If the neutrinos with definite masses $v_{i}, i=1,2,3$, are Majorana particles, the 3-neutrino mixing matrix contains two additional Majorana CPV phases [51]. However, the flavour neutrino oscillation probabilities $P\left(v_{l} \rightarrow v_{l^{\prime}}\right)$ and $P\left(\bar{v}_{l} \rightarrow \bar{v}_{l^{\prime}}\right), l, l^{\prime}=e, \mu, \tau$, do not depend on the Majorana phases[51,63]. The Majorana phases can play important role, e.g, in $|\Delta L|=2$ processes like neutrinoless double beta $\left((\beta \beta)_{0 v^{-}}\right)$decay $(A, Z) \rightarrow(A, Z+2)+e^{-}+e^{-}, L$ being the total lepton charge, in which the Majorana nature of massive neutrinos $v_{i}$ manifests itself (see, e.g, Refs. [52,53,64]).

Our interest in the CPV phases present in the neutrino mixing matrix is stimulated also by the intriguing possibility that the Dirac phase and/or the Majorana phases in $U_{\text {PMNS }}$ can provide the $\mathrm{CP}$ violation necessary for the generation of the observed baryon asymmetry of the Universe (BAU) $[65,66]$ (for specific models in which this possibility is realised see, e.g., [67-70]; for a recent review see [71]). More specifically, if, e.g., all CP violation necessary for the generation of BAU is due to the Dirac phase $\delta$, which is possible within the "flavoured" leptogenesis scenario [72,73] of generation of baryon asymmetry, a necessary condition for reproducing the observed BAU in this scenario (with hierarchical heavy Majorana neutrinos) is [65] $\left|\sin \theta_{13} \sin \delta\right| \gtrsim 0.09$. This condition is comfortably compatible with the measured value of $\sin \theta_{13}$ and with the best fit value of $\delta \sim 3 \pi / 2$.

The sign of $\Delta m_{31(32)}^{2}$ cannot be directly and unambiguously determined from the existing data. ${ }^{5}$ In the case of 3 neutrino mixing, the two possible signs of $\Delta m_{31 \text { (32) }}^{2}$ correspond to two types of neutrino mass spectrum. In the convention of numbering of neutrinos $v_{j}$ employed by us the two spectra read:

(i) spectrum with normal ordering (NO): $m_{1}<m_{2}<$ $m_{3}, \Delta m_{31(32)}^{2}>0, \Delta m_{21}^{2}>0, m_{2(3)}=\left(m_{1}^{2}+\right.$ $\left.\Delta m_{21(31)^{2}}^{2}\right)^{\frac{1}{2}}$

(ii) spectrum with inverted ordering (IO): $m_{3}<m_{1}<$ $m_{2}, \Delta m_{32(31)}^{2}<0, \Delta m_{21}^{2}>0, m_{2}=\left(m_{3}^{2}+\Delta m_{23}^{2}\right)^{\frac{1}{2}}$, $m_{1}=\left(m_{3}^{2}+\Delta m_{23}^{2}-\Delta m_{21}^{2}\right)^{\frac{1}{2}}$.

Depending on the values of the lightest neutrino mass, $\min \left(m_{j}\right)$, the neutrino mass spectrum can also be:

\footnotetext{
${ }^{5}$ In the recent analysis of the global neutrino oscillation data performed in [58] it was found that the case of $\Delta m_{31(32)}^{2}<0$ is disfavored at $3.1 \sigma$ with respect to the case of $\Delta m_{31(32)}^{2}>0$.
}

(a) Normal Hierarchical (NH): $m_{1} \ll m_{2}<m_{3}, m_{2} \cong$ $\left(\Delta m_{21}^{2}\right)^{\frac{1}{2}} \cong 8.6 \times 10^{-3} \mathrm{eV}, m_{3} \cong\left(\Delta m_{31}^{2}\right)^{\frac{1}{2}} \cong 0.0506$ $\mathrm{eV}$; or

(b) Inverted Hierarchical (IH): $m_{3} \ll m_{2}<m_{2}, m_{1} \cong$ $\left(\left|\Delta m_{32}^{2}\right|-\Delta m_{21}^{2}\right)^{\frac{1}{2}} \cong 0.0497 \mathrm{eV}, m_{2} \cong\left(\left|\Delta m_{32}^{2}\right|\right)^{\frac{1}{2}} \cong$ $0.0504 \mathrm{eV}$; or

(c) Quasi-Degenerate $(Q D): m_{1} \cong m_{2} \cong m_{3} \cong m_{0}$, $m_{j}^{2} \gg\left|\Delta m_{31(32)}^{2}\right|, m_{0} \gtrsim 0.10 \mathrm{eV}$.

All three types of spectrum are compatible with the constraints on the absolute scale of neutrino masses. Determining the type of neutrino mass spectrum is one of the main goals of the future experiments in the field of neutrino physics 6 (see, e.g., Refs. [1,74,86-90]).

Data on the absolute neutrino mass scale (or on $\min \left(m_{j}\right)$ ) can be obtained, e.g., from measurements of the spectrum of electrons near the end point in ${ }^{3} \mathrm{H} \beta$-decay experiments [9194] and from cosmological and astrophysical observations. The most stringent upper bound on the $\bar{v}_{e}$ mass was reported by the Troitzk [95] experiment:

$m_{\bar{v}_{e}}<2.05 \mathrm{eV}$ at $95 \%$ C.L.

Similar result was obtained in the Mainz experiment [93] : $m_{\bar{v}_{e}}<2.3 \mathrm{eV}$ at $95 \% \mathrm{CL}$. We have $m_{\bar{v}_{e}} \cong m_{1,2,3}$ in the case of QD spectrum. The KATRIN experiment [96], which was commissioned on June 11, 2018, is designed to reach sensitivity of $m_{\bar{v}_{e}} \sim 0.20 \mathrm{eV}$, i.e., to probe the region of the QD spectrum.

Constraints on the sum of the neutrino masses can be obtained from cosmological and astrophysical data (see, e.g., Ref. [97]). Depending on the model complexity and the input data used one typically obtains [97]: $\sum_{j} m_{j} \lesssim(0.3-1.3)$ eV, 95\% C.L. Assuming the existence of three light massive neutrinos and the validity of the $\Lambda$ CDM (Cold Dark Matter) model, and using their data on the CMB temperature power spectrum anisotropies, polarisation, on gravitational lensing effects and the low $l \mathrm{CMB}$ polarization spectrum data (the low $\mathrm{P}$ data), etc. the Planck Collaboration reported an updated upper limit on the sum of the neutrino masses $[98,99]$, which, depending on the data-set used, varies in the interval: $\sum_{j} m_{j}<(0.340-0.715) \mathrm{eV}, 95 \%$ C.L. Adding data on Baryon Acoustic Oscillations (BAO) lowers the limit to $[98,99]$ :

$\sum_{j} m_{j}<0.170 \mathrm{eV}, \quad 95 \%$ C.L.

In spite of the remarkable progress made in the last 19 years in establishing the existence of neutrino oscillations

\footnotetext{
${ }^{6}$ For a brief discussion of experiments which can provide data on the type of neutrino mass spectrum see, e.g., Ref. [74]; for some specific proposals see, e.g., Refs. [75-85].
} 
caused by non-zero neutrino masses and neutrino mixing and in measuring the 3-neutrino oscillation parameters, one has to admit that we are still completely ignorant about the fundamental aspects of neutrino mixing. We do not know whether the massive neutrinos are Dirac or Majorana particles, what is the neutrino mass ordering, what is the status of the CP symmetry in the lepton sector and what is the absolute neutrino mass scale (i.e., the lightest neutrino mass). Determining the nature - Dirac or Majorana - of massive neutrinos, the type of spectrum the neutrino masses obey, establishing the status of the CP symmetry in the lepton sector and determining the absolute neutrino mass scale are among the highest priority goals of the programme of future experimental research in neutrino physics (see, e.g., [1,86-90,100-102]), which extends beyond 2030. The principal goal of the theoretical studies in this field is the understanding at a fundamental level the mechanism giving rise to neutrino masses and mixing and to $L_{l}$-non-conservation. Are the observed patterns of $\nu$-mixing and of $\Delta m_{21,31}^{2}$ related to the existence of a new fundamental symmetry of particle interactions? Is there any relation between quark mixing and neutrino mixing? What is the physical origin of CPV phases in the neutrino mixing matrix $U$ ? Is there any relation (correlation) between the (values of) CPV phases and mixing angles in $U$ ? Progress in the theory of neutrino mixing might also lead, in particular, to a better understanding of the mechanism of generation of baryon asymmetry of the Universe.

\section{Origins of the pattern of neutrino mixing: the discrete symmetry approach}

\subsection{The general framework}

The observed pattern of neutrino mixing in the reference 3neutrino mixing scheme we are going to consider in what follows is characterised, as we have seen, by two large mixing angles $\theta_{12}$ and $\theta_{23}$, and one small mixing angle $\theta_{13}$ : $\theta_{12} \cong 33^{\circ}, \theta_{23} \cong 45^{\circ} \pm 6^{\circ}$ and $\theta_{13} \cong 8.4^{\circ}$. These values can naturally be explained by extending the Standard Model (SM) with a flavour symmetry corresponding to a non-Abelian discrete (finite) group $G_{f}$. This symmetry is supposed to exist at some high-energy scale and to be broken at lower energies to residual symmetries of the charged lepton and neutrino sectors, described respectively by subgroups $G_{e}$ and $G_{v}$ of $G_{f}$. Flavour symmetry groups $G_{f}$ that have been used in this approach to neutrino mixing and lepton flavour include $A_{4}[103,104], S_{4}[105-107], T^{\prime}[108-$ 112], $A_{5}$ [113,114], $D_{n}$ (with $\left.n=10,12\right)$ [115-117], $\Delta(27)$ $[118,119]$, the series $\Delta\left(6 n^{2}\right)[120-124]$, to name several ${ }^{7}$

\footnotetext{
7 Some of the groups $T^{\prime}, A_{5}$, etc. can be and have been used also for a unified description of the quark and lepton flavours, see, e.g., Refs. $[36,108-112,125-130]$ and references quoted therein.
}

(see, e.g., Ref. [10] for definitions of these groups and discussion of their properties. ${ }^{8}$ ) The numbers of elements, of generators and of irreducible representations of the groups $S_{4}, A_{4}, T^{\prime}, A_{5}, D_{10}$ and $D_{12}$ are given in Table 2. In what concerns the group $S_{4}$, it is well known that $S_{4}$ can be generated by two transformations, $S$ and $T$ (see, e.g.,[10]). However, in the context of non-Abelian discrete symmetry approach to neutrino mixing it often proves convenient to use the three generators $S, T$ and $U$ of $S_{4}$, indicated in Table 2, and these generators are widely used in the literature on the subject (see, e.g., the review article [11]). We will use the two generator formalism for the group $S_{4}$ in the analysis performed in Sect. 4.2.

The choice of the non-Abelian discrete groups $A_{4}, S_{4}, T^{\prime}$, $A_{5}$, etc. is related, in particular, to the fact that they describe symmetries with respect to rotations on fixed large mixing angles and, correspondingly, lead to values of the neutrino mixing angles $\theta_{12}$ and $\theta_{23}$, which can differ from the measured values at most by sub-leading perturbative corrections, with $\theta_{13}$ typically (but not universally) predicted to be zero. The requisite corrections can most naturally be provided by the unitary matrix $U_{e}$ which originates from the diagonalisation of the charged lepton mass term and enters into the expression of the PMNS neutrino mixing matrix (see, e.g., [131-133] and references quoted therein):

$U_{\mathrm{PMNS}}=U_{e}^{\dagger} U_{\nu}$

where $U_{v}$ is a unitary matrix coming from the diagonalisation of the neutrino mass term. More specifically, $U_{e}$ diagonalises the product $M_{e} M_{e}^{\dagger}$, where $M_{e}$ is the charged lepton mass matrix in the charged lepton mass term $\mathcal{L}_{\ell}(x)$ (written in the left-right convention):

$$
\begin{aligned}
\mathcal{L}_{\ell}(x) & =-\overline{\tilde{l}_{L}(x)}\left(M_{e}\right) \tilde{l l}^{\prime} \tilde{l}_{R}^{\prime}(x)+\text { h.c. }, \\
U_{e}^{\dagger} M_{e} M_{e}^{\dagger} U_{e} & =\operatorname{diag}\left(m_{e}^{2}, m_{\mu}^{2}, m_{\tau}^{2}\right),
\end{aligned}
$$

$\tilde{l}_{L}(x)$ and $\tilde{l}_{R}^{\prime}(x), \tilde{l}, \tilde{l}^{\prime}=\tilde{e}, \tilde{\mu}, \tilde{\tau}$, being respectively the $S U(2)$ doublet and singlet left-handed (LH) and right-handed $(\mathrm{RH})$ components of the charged lepton fields in the basis in which the charged lepton mass term $\mathcal{L}_{\ell}(x)$ is not diagonal, while $m_{e}, m_{\mu}$ and $m_{\tau}$ are the masses of the charged leptons. ${ }^{9}$ In certain classes of models, however, $U_{e}$ coincides with the unit $3 \times 3$ matrix and the requisite corrections are incorporated in a factor contained in the matrix $U_{v}$ (see, e.g., [11,134]).

\footnotetext{
${ }_{8} A_{4}$ is the group of even permutations of 4 objects and the symmetry group of the regular tetrahedron. $S_{4}$ is the group of permutations of 4 objects and the symmetry group of the cube. $T^{\prime}$ is the double covering group of $A_{4} . A_{5}$ is the icosahedron symmetry group of even permutations of five objects, etc. All these groups are subgroups of the group $S U(3)$.

9 The LH components of the fields of the electron, muon, and tauon, $l_{L}(x), l=e, \mu, \tau$, are related to the fields $\tilde{l}_{L}(x)$ via the matrix $U_{e}$ : $l_{L}(x)=\left(U_{e}^{\dagger}\right)_{l l} \tilde{l}_{L}(x)$.
} 
Table 2 Number of elements, generators and irreducible representations of some discrete groups

\begin{tabular}{llll}
\hline Group & Number of elements & Generators & Irreducible representations \\
\hline$S_{4}$ & 24 & $S, T(U)$ & $1,1^{\prime}, 2,3,3^{\prime}$ \\
$A_{4}$ & 12 & $S, T$ & $1,1^{\prime}, 1^{\prime \prime}, 3$ \\
$T^{\prime}$ & 24 & $S, T(R)$ & $1,1^{\prime}, 1^{\prime \prime}, 2,2^{\prime}, 2^{\prime \prime}, 3$ \\
$A_{5}$ & 60 & $\tilde{S}, \tilde{T}$ & $1,3,3^{\prime}, 4,5$ \\
$D_{10}$ & 20 & $A, B$ & $1_{1}, 1_{2}, 1_{3}, 1_{4}, 2_{1}, 2_{2}, 2_{3}, 2_{4}$ \\
$D_{12}$ & 24 & $\tilde{A}, \tilde{B}$ & $1_{1}, 1_{2}, 1_{3}, 1_{4}, 2_{1}, 2_{2}, 2_{3}, 2_{4}, 2_{5}$ \\
\hline
\end{tabular}

We shall assume in what follows that the weak-eigenstate neutrino fields (in the basis in which charged lepton mass term is not diagonal), $v_{\tilde{e}}(x), v_{\tilde{\mu}}(x)$ and $v_{\tilde{\tau}}(x)$, possess a Majorana mass term, $\mathcal{L}_{M}^{\nu}(x)$, and thus the neutrinos with definite mass $v_{1}, v_{2}$ and $v_{3}$, are Majorana particles. In this case $U_{v}$ diagonalises the neutrino Majorana mass matrix $M_{\nu}$ :

$$
\begin{aligned}
\mathcal{L}_{M}^{v}(x) & =\frac{1}{2} v_{\tilde{l}^{\prime} L}^{\mathrm{T}}(x) C^{-1} M_{\nu \tilde{l} \tilde{l}^{\prime}} v_{\tilde{l} L}(x)+\text { h.c. }, \\
C^{-1} \gamma_{\alpha} C & =-\gamma_{\alpha}^{\mathrm{T}}, \\
U_{v}^{\mathrm{T}} M_{v} U_{v} & =\operatorname{diag}\left(m_{1}, m_{2}, m_{3}\right),
\end{aligned}
$$

where $C$ is the charge conjugation matrix (see, e.g., [52]). It should be noted, however, that the approach to neutrino mixing we are discussing can be employed also if $v_{1,2,3}$ are Dirac fermions (see, e.g., [14]), e.g., when the theory contains right-handed neutrino fields $v_{\tilde{l} R}(x)$ which form a Dirac mass term with the LH neutrino fields $v_{\tilde{l}^{\prime} L}(x) \tilde{l}, \tilde{l}^{\prime}=\tilde{e}, \tilde{\mu}, \tilde{\tau}$, and ${ }^{10}$ the total lepton charge $L=L_{e}+L_{\mu}+L_{\tau}$ is conserved.

In the approach under discussion it is standardly assumed that the LH neutrino fields, $v_{\tilde{l} L}(x)$, and the LH components of the charged lepton fields (in the basis in which charged lepton mass term is not diagonal) $\tilde{l}_{L}(x)$, which form an $S U(2)_{L}$ doublet in the Standard Theory, are assigned to the same r-dimensional irreducible unitary representation $\rho_{r}\left(g_{f}\right)$ of the Group $G_{f}, g_{f}$ being an element of $G_{f}$. Thus, under the action of $G_{f}, v_{\tilde{l} L}(x)$ and $\tilde{l}_{L}(x)$ transform as follows:

$$
\begin{aligned}
& v_{\tilde{l} L}(x) \stackrel{G_{f}}{\longrightarrow}\left(\rho_{r}\left(g_{f}\right)\right)_{\tilde{l} \tilde{l}^{\prime}} v_{\tilde{l}^{\prime} L}(x), \quad g_{f} \in G_{f}, \\
& \tilde{l}_{L}(x) \stackrel{G_{f}}{\longrightarrow}\left(\rho_{r}\left(g_{f}\right)\right)_{\tilde{l} l^{\prime}} \tilde{l}_{L}^{\prime}(x), \quad \tilde{l}=\tilde{e}, \tilde{\mu}, \tilde{\tau} .
\end{aligned}
$$

In the cases of $G_{f}=A_{4}, S_{4}, T^{\prime}$ and $A_{5}$, which possess 3dimensional irreducible representations, $\rho\left(g_{f}\right)$ is standardly taken to be a 3 -dimensional irreducible unitary representation 3, $\rho_{r}\left(g_{f}\right)=\rho_{3}\left(g_{f}\right)$. This is equivalent to the assumption of

\footnotetext{
10 The neutrino Dirac mass term in question originates [135] from an $S U(2)_{L} \times U(1)_{Y_{w}}$ invariant Yukawa-type term in the Lagrangian after the spontaneous breaking of the Standard Theory $S U(2)_{L} \times U(1)_{Y_{w}}$ symmetry.
}

unification of the three lepton families at some high energy scale. We are going to consider this choice in what follows. ${ }^{11}$

At low energies the flavour symmetry $G_{f}$ has necessarily to be broken so that the electron, muon and tauon as well as the three neutrinos with definite mass $v_{1}, v_{2}$ and $v_{3}$, can get different masses. The breaking of $G_{f}$ is realised in specific models by scalar "flavon" fields, which are singlets with respect to the Standard Theory gauge group but transform under certain irreducible representations of $G_{f}$ and acquire non-zero vacuum expectation values (VEVs), thus breaking $G_{f}$ spontaneously. The breaking of the flavour symmetry $G_{f}$ can leave certain subgroups of $G_{f}, G_{e}$ and $G_{\nu}$, unbroken in the charged lepton and neutrino sectors. The unbroken symmetries $G_{e} \in G_{f}$ and $G_{v} \in G_{f}$ are residual symmetries of the charged lepton and neutrino mass matrices.

The existence of a residual symmetry $G_{e} \in G_{f}$ in the charged lepton sector implies that $M_{e} M_{e}^{\dagger}$ is invariant with respect to the action of $G_{e}$ on the LH components of the charged lepton fields $\tilde{l}_{L}(x), \tilde{l}=\tilde{e}, \tilde{\mu}, \tilde{\tau}$ :

$\rho_{r}\left(g_{e}\right)^{\dagger} M_{e} M_{e}^{\dagger} \rho_{r}\left(g_{e}\right)=M_{e} M_{e}^{\dagger}$,

where $g_{e}$ is an element of $G_{e}$ and $\rho_{r}\left(g_{e}\right)$ gives the action of $G_{e}$ on $\tilde{l}_{L}(x)$.

Similarly, if $G_{v}$ is the residual symmetry of the neutrino Majorana mass matrix $M_{v}$ one has:

$\rho_{r}\left(g_{\nu}\right)^{T} M_{\nu} \rho_{r}\left(g_{\nu}\right)=M_{\nu}$

where $g_{v}$ is an element of $G_{v}$ and $\rho_{r}\left(g_{v}\right)$ determines the action of $G_{v}$ on $v_{\tilde{l} L}(x), \tilde{l}=\tilde{e}, \tilde{\mu}, \tilde{\tau}$. From Eq. (16) we get:

$\rho_{r}\left(g_{v}\right)^{\dagger} M_{v}^{\dagger} M_{v} \rho_{r}\left(g_{v}\right)=M_{v}^{\dagger} M_{v}$.

It follows from Eqs. (15) and (17) that $M_{e} M_{e}^{\dagger}$ commutes with $\rho_{r}\left(g_{e}\right)$, while $M_{v}^{\dagger} M_{v}$ commutes with $\rho_{r}\left(g_{v}\right)$. This implies that $M_{e} M_{e}^{\dagger}$ and $\rho_{r}\left(g_{e}\right)$ are diagonalised with one and

${ }^{11}$ In specific models the choice $\rho_{r}\left(g_{f}\right)=\rho_{3}\left(g_{f}\right)$ is usually accompanied by the assumption that $\tilde{e}_{R}(x), \tilde{\mu}_{R}(x)$ and $\tilde{\tau}_{R}(x)$ transform as singlet irreducible representations of $G_{f}$ (see, e.g., [11]). 
the same matrix $U_{e}$, and that similarly, $M_{v}^{\dagger} M_{v}$ and $\rho_{r}\left(g_{v}\right)$ are diagonalised by the same matrix $U_{v}^{\circ}$ :

$$
\begin{aligned}
U_{e}^{\dagger} \rho_{r}\left(g_{e}\right) U_{e} & =\rho_{r}^{\operatorname{diag}}\left(g_{e}\right), \\
\left(U_{v}^{\circ}\right)^{\dagger} \rho_{r}\left(g_{v}\right) U_{v}^{\circ} & =\rho_{r}^{\operatorname{diag}}\left(g_{v}\right) .
\end{aligned}
$$

Given $G_{f}, \rho_{r}\left(g_{f}\right)$, and non-trivial $G_{e}, \rho_{r}\left(g_{e}\right)$ is uniquely determined. As a consequence, the matrix $U_{e}$ diagonalising $\rho_{r}\left(g_{e}\right)$ (and $M_{e} M_{e}^{\dagger}$ ), which enters into the expression for the PMNS matrix $U$, is either completely determined or significantly constrained. ${ }^{12}$ Similarly, for given $G_{f}, \rho_{r}\left(g_{f}\right)$, and non-trivial $G_{v}$, the matrix $U_{v}^{\circ}$ disgonalising $\rho_{r}\left(g_{v}\right)$ (and $M_{v}^{\dagger} M_{v}$ ) will either be completely determined or strongly constrained. One can show that the matrix $U_{v}$ diagonalising the neutrino Majorana mass matrix $M_{v}$ and the matrix $U_{v}^{\circ}$ diagonalising $M_{v}^{\dagger} M_{v}$ are related, in general, in the following way:

$U_{\nu}=U_{v}^{\circ} P^{\circ}, \quad P^{\circ}=\operatorname{diag}\left(1, e^{i \frac{\xi_{21}}{2}}, e^{i \frac{\xi_{31}}{2}}\right)$.

The phases $\xi_{21}$ and $\xi_{31}$ contribute respectively to the Majorana phases $\alpha_{21}$ and $\alpha_{31}$ of the PMNS matrix (see Eq. (2)).

Thus, within the discussed approach the PMNS neutrino mixing matrix $U=U_{e}^{\dagger} U_{v}$ is either completely determined or else has a constrained form. The form of $U$ one obtains depends on the choices of $G_{f}, \rho_{r}\left(g_{f}\right), G_{e}$ and $G_{\nu}$.

It should be clear from the preceding discussion that the residual symmetries $G_{e}$ and $G_{v}$, in particular, play a crucial role in obtaining a specific form of the PMNS matrix. If, in particular, $G_{e} \equiv G_{v}$, we would have $U_{e}=U_{v}^{\circ}$ and the PMNS matrix will be trivial, which is ruled out by the data.

The largest possible exact symmetry of the charged lepton Dirac mass term $\mathcal{L}_{\ell}(x)$ (mass matrix $M_{\ell}$ ) is $U(1) \times U(1) \times$ $U(1)$. The largest possible exact symmetry of the neutrino Majorana mass term $\mathcal{L}_{M}^{v}(x)$, with mass matrix $M_{v}$ having three non-zero non-degenerate eigenvalues, is $Z_{2} \times Z_{2} \times Z_{2}$. Making the standardly used simplifying assumption that $G_{f}$ is a subgroup of $S U(3)$, the largest possible symmetries of $\mathcal{L}_{\ell}(x)$ and $\mathcal{L}_{M}^{v}(x)$ reduce to $U(1) \times U(1)$ and $Z_{2} \times Z_{2}$ owing to the $S U$ (3) determinant condition. The residual symmetry group $G_{e}$ should be a subgroup of $U(1) \times U(1)$, while $G_{v}$ should be contained in $Z_{2} \times Z_{2}(U(1) \times U(1))$ in the case of massive Majorana (Dirac) neutrinos. Thus, $G_{e}$ and $G_{v}$ should be Abelian groups.

It follows from the preceding discussion that the possible discrete symmetries $G_{e}$ of the charged lepton mass term leaving $M_{e} M_{e}^{\dagger}$ invariant are: (i) $G_{e}=Z_{n}$, with integer $n \geq 2$, or (ii) $Z_{m} \times Z_{k}$, with integers $m, k \geq 2$. The maximal symmetry $G_{v}$ of the Majorana mass term of the LH flavour neutrino fields $v_{\tilde{l} L}(x)$ is the $Z_{2} \times Z_{2}$ (sometimes referred to as

\footnotetext{
12 Obviously, if $G_{e}$ is trivial consisting just of the unit element of $G_{f}$, i.e., if $G_{f}$ is completely broken in the charged lepton sector, $U_{e}$ would not be constrained.
}

the Klein four group) symmetry. $G_{\nu}$ can obviously be just $Z_{2}$. These two possible types of $G_{v}$ are associated with two approaches in constructing realistic models of lepton flavour: the direct approach with $G_{v}=Z_{2} \times Z_{2}$ and the semi-direct approach with $G_{v}=Z_{2}$. Since the neutrino Majorana mass term (mass matrix $M_{\nu}$ ) possesses always a $Z_{2} \times Z_{2}$ symmetry, the second $Z_{2}$ factor appears accidentally in models employing the semi-direct approach. The symmetry $G_{f}$ might be completely broken by the neutrino Majorana mass term $\mathcal{L}_{M}^{v}(x)$, i.e., the $Z_{2} \times Z_{2}$ group of symmetry of $\mathcal{L}_{M}^{v}(x)$ might not necessarily be a subgroup of $G_{f}$. This corresponds to the so-called indirect approach in lepton flavour model building.

The group $A_{4}$, for example, has three subgroups $Z_{2}$, four subgroups $Z_{3}$ and one subgroup $Z_{2} \times Z_{2}$, while $S_{4}$ has nine $Z_{2}$, four $Z_{3}$, three $Z_{4}$ and four $Z_{2} \times Z_{2}$ subgroups. The bigger groups $T^{\prime}, A_{5}$, etc. all have a certain number of $Z_{2}$, $Z_{3}, Z_{2} \times Z_{2}$, etc. subgroups. ${ }^{13}$

As we have indicated in the Sect. 1, one of the main characteristics of the discussed approach to neutrino mixing based on discrete flavour symmetries is that it leads to certain specific predictions for the values of, and/or correlations between, the low-energy neutrino mixing parameters, which can be tested experimentally. These predictions depend on the chosen $G_{f}, \rho\left(g_{f}\right), G_{e}$ and $G_{\nu}$. We give a few examples $[11-14,22,25,30,33,37,136]$.

I. In a large class of models one gets $\sin ^{2} \theta_{23}=0.5$.

II. In different class of models one finds that the values of $\sin ^{2} \theta_{23}$ and $\sin ^{2} \theta_{13}$ are correlated: $\sin ^{2} \theta_{23}=0.5(1 \mp$ $\left.\sin ^{2} \theta_{13}+O\left(\sin ^{4} \theta_{13}\right)\right)$.

III. In certain models $\sin ^{2} \theta_{23}$ is predicted to have specific values which differ significantly from those in cases I and II [13]: $\sin ^{2} \theta_{23}=0.455$; or 0.463 ; or 0.537 ; or 0.545 , the uncertainties in these predictions being insignificant.

IV. Certain class of models predict a correlation between the values of $\sin ^{2} \theta_{12}$ and $\sin ^{2} \theta_{13}: \sin ^{2} \theta_{12}=$ $1 /\left(3 \cos ^{2} \theta_{13}\right)=\left(1+\sin ^{2} \theta_{13}+O\left(\sin ^{4} \theta_{13}\right)\right) / 3 \cong$ 0.340 , where we have used the b.f.v. of $\sin ^{2} \theta_{13}$.

V. In another class of models one still finds a correlation between the values of $\sin ^{2} \theta_{12}$ and $\sin ^{2} \theta_{13}$, which, however, differs from that in Case IV: $\sin ^{2} \theta_{12}=$ $\left(1-3 \sin ^{2} \theta_{13}\right) /\left(3 \cos ^{2} \theta_{13}\right)=\left(1-2 \sin ^{2} \theta_{13}+\right.$ $\left.O\left(\sin ^{4} \theta_{13}\right)\right) / 3 \cong 0.319$, where we have used again the b.f.v. of $\sin ^{2} \theta_{13}$.

VI. In large classes of models in which the elements of the PMNS matrix are predicted to be functions of just one real continuous free parameter ("one-parameter mod-

\footnotetext{
${ }^{13}$ For complete list of the subgroups of the groups $T^{\prime}, A_{5}, \Delta\left(6 n^{2}\right)$ and of the larger groups employed in the discrete flavour symmetry approach to neutrino mixing see, e.g., Ref. [10].
} 
els"), the Dirac and the Majorana CPV phases have "trivial" CP conserving values 0 or $\pi$. In certain oneparameter schemes, however, the Dirac phase $\delta=\pi / 2$ or $3 \pi / 2$.

VII. In theories/models in which the elements of the PMNS matrix are functions of two (angle) or three (two angle and one phase) parameters, the Dirac phase $\delta$ satisfies a sum rule by which $\cos \delta$ is expressed in terms of the three neutrino mixing angles $\theta_{12}, \theta_{23}, \theta_{13}$ and one (or more) fixed (known) parameters $\theta^{v}$ which depend on the discrete symmetry $G_{f}$ employed and on the residual symmetries $G_{e}$ and $G_{v}$ [12-14]:

$\cos \delta=\cos \delta\left(\theta_{12}, \theta_{23}, \theta_{13} ; \theta^{v}\right)$.

In these cases the $J_{C P}$ factor which determines the magnitude of $\mathrm{CP}$ violation effects in neutrino oscillations, is also completely determined by the values of the three neutrino mixing angles and the symmetry parameter(s) $\theta_{\nu}:$

$$
J_{C P}=J_{C P}\left(\theta_{12}, \theta_{23}, \theta_{13}, \delta\right)=J_{C P}\left(\theta_{12}, \theta_{23}, \theta_{13} ; \theta^{v}\right) \text {. }
$$

If in the model considered a correlation of the type corresponding to Case II (case IV or case V) takes place, $\theta_{23}\left(\theta_{12}\right)$ in the sum rule for $\cos \delta$ and the expression for the $J_{C P}$ factor has to be expressed in terms of $\theta_{13}$ using the correlation.

The predictions listed above, and therefore the respective models, can be and will be tested in the currently running (T2K [100] and NOvA [137]) and planned future (JUNO [86], T2HK [101], T2HKK [102] and DUNE [89]) experiments.

As an illustration of the preceding discussion we will consider first the example of the tri-bimaximal mixing as an underlying symmetry form of the matrix $U_{v}\left(U_{v}^{\circ}\right)$.

\subsection{Symmetry forms of $U_{v}$ : tri-bimaximal mixing}

Consider the case of $G_{f}=S_{4}$, i.e., the group of permutations of four objects. $S_{4}$ is isomorphic to the group of rotational symmetries of the cube. It has 24 elements, two singlet, one doublet and two triplet irreducible representations. As was indicated earlier, we will assume that $\rho_{r}\left(g_{f}\right)=\rho_{3}\left(g_{f}\right)$, i.e., that $v_{\tilde{l}}(x)$ and $\tilde{l}_{L}(x)$ transform under one of the two 3-dimensional irreducible unitary representations of $S_{4}$. In what follows, with the exception of Sect. 4.2, we will work with the three generators of the group $S_{4}, S, T$ and $U$. These generators satisfy the following presentation rules (see, e.g., [10]):

$$
S^{2}=T^{3}=(S T)^{3}=U^{2}=(T U)^{2}=(S U)^{2}=(S T U)^{4}=\mathbf{1},
$$

1 being the unit (identity) element of $S_{4}$, i.e., it is the $3 \times 3$ unit matrix in the case of the triplet representations of $S$, $T$ and $U$. In what follows we will use the basis [138] in which $S, T$ and $U$ have the following form in the two triplet representations ${ }^{14}$ :

$S=\frac{1}{3}\left(\begin{array}{ccc}-1 & 2 & 2 \\ 2 & -1 & 2 \\ 2 & 2 & -1\end{array}\right), \quad T=\left(\begin{array}{ccc}1 & 0 & 0 \\ 0 & \omega^{2} & 0 \\ 0 & 0 & \omega\end{array}\right) \quad$ and

$U=\mp\left(\begin{array}{lll}1 & 0 & 0 \\ 0 & 0 & 1 \\ 0 & 1 & 0\end{array}\right)$,

where $\omega=e^{2 \pi i / 3}$. For simplicity we use the same notation ( $S, T$ and $U$ ) for the generators and their 3-dimensional representation matrices.

Assume next that [139] (see also, e.g., [11,14])

$G_{e}=Z_{3}^{T}=\left\{1, T, T^{2}\right\}, \quad G_{v}=Z_{2}^{S} \times Z_{2}^{U}=\{1, S, U, S U\}$,

where $Z_{3}^{T}$ and $Z_{2}^{S} \times Z_{2}^{U}$ are two specific $Z_{3}$ and $Z_{2} \times Z_{2}$ subgroups of $S_{4} \cdot{ }^{15}$ In this case we have, in particular: $\rho_{3}\left(g_{e}\right)=$ $1, T, T^{2}, T$ being the diagonal matrix given in Eq. (24). As a consequence, $U_{e}$, which diagonalises $\rho_{3}\left(g_{e}\right)=T$, is just a diagonal phase matrix, whose phases are unphysical (they can be absorbed by the charged lepton fields in the weak charged lepton current of the weak interaction Lagrangian), while $M_{e}$ is a diagonal matrix with the masses of the electron, muon and tauon as diagonal elements.

It follows from Eq. (23) that $\rho_{3}\left(g_{v}\right)=S$ and $\rho_{3}\left(g_{v}^{\prime}\right)=U$ commute. In the triplet representation of the generators of $S_{4}$ employed by us, Eq. (24), $S$ and $U$ are real symmetric matrices. Thus, they are diagonalised by a real orthogonal matrix. The matrix which diagonalises both $\rho_{3}\left(g_{v}\right)=S$ and $\rho_{3}\left(g_{v}^{\prime}\right)=U$, with $S$ and $U$ given in Eq. (24), is the orthogonal tri-bimaximal (TBM) mixing matrix [140-142]:

$U_{v}^{\circ}=V_{\mathrm{TBM}}=\left(\begin{array}{ccc}\sqrt{\frac{2}{3}} & \frac{1}{\sqrt{3}} & 0 \\ -\frac{1}{\sqrt{6}} & \frac{1}{\sqrt{3}} & -\frac{1}{\sqrt{2}} \\ -\frac{1}{\sqrt{6}} & \frac{1}{\sqrt{3}} & \frac{1}{\sqrt{2}}\end{array}\right)$.

\footnotetext{
14 As can be shown, the results one obtains for the form of the PMNS matrix are independent of the chosen basis for the generators of the discrete symmetry group $G_{f}$.

$15 S$ and $U$ are order two elements of $S_{4}$ (since $S^{2}=U^{2}=\mathbf{1}$ ) and they commute. Correspondingly, $Z_{2}^{S} \times Z_{2}^{U}=\{1, S, U, S U\}$ is a subgroup of $S_{4}$. Similarly, $T$ is order 3 element of $S_{4}$ and $Z_{3}^{T}=\left\{1, T, T^{2}\right\}$ is a subgroup of $S_{4}$.
} 
Indeed, it is not difficult to check that

$$
\begin{aligned}
V_{\mathrm{TBM}}^{\dagger} S V_{\mathrm{TBM}} & =\operatorname{diag}(-1,1,-1), \\
V_{\mathrm{TBM}}^{\dagger} U V_{\mathrm{TBM}} & = \pm \operatorname{diag}(1,1,-1) .
\end{aligned}
$$

Thus, in the discussed case of $S_{4}$ symmetry and residual symmetries $G_{e}=Z_{3}^{T}$ and $G_{v}=Z_{2}^{S} \times Z_{2}^{U}$, the PMNS matrix has the TBM form [139], $U=U_{v}^{\circ} P^{\circ}=V_{\text {TBM }} P^{\circ}$. We can cast $V_{\mathrm{TBM}}$ in the form:

$$
\begin{aligned}
V_{\mathrm{TBM}} & =R_{23}\left(\theta_{23}^{v}\right) R_{13}\left(\theta_{13}^{v}\right) R_{12}\left(\theta_{12}^{v}\right), \theta_{23}^{v}=-\pi / 4, \\
\theta_{13}^{v} & =0, \theta_{12}^{v}=\sin ^{-1} \frac{1}{\sqrt{3}},
\end{aligned}
$$

where $R_{23}\left(\theta_{23}^{v}\right), R_{13}\left(\theta_{13}^{v}\right)$ and $R_{12}\left(\theta_{12}^{v}\right)$ are $3 \times 3$ orthogonal matrices describing rotations in the $2-3,1-3$ and 1-2 planes, respectively. We see that in the case of the TBM symmetry form we have $\sin ^{2} \theta_{12}^{v}=1 / 3, \sin ^{2} \theta_{23}^{v}=1 / 2$ and $\sin ^{2} \theta_{13}^{v}=$ 0 . Without additional corrections leading to $\theta_{13} \cong 0.15 \neq 0$, the TBM symmetry from of the PMNS matrix is ruled out by the data.

We will consider next two cases of realistic models based on the flavour symmetries $G_{f}=A_{4}$ and $G_{f}=S_{4}$, in which the corrections to the underlying symmetry form of the PMNS matrix are obtained by "decreasing" the residual symmetry $G_{v}$ from $Z_{2} \times Z_{2}$ symmetry to $Z_{2}$,

\subsection{Neutrino mixing from $A_{4}$ symmetry}

The group $A_{4}$ has two generators $S$ and $T$, which satisfy the presentation rules given in Eq. (23). In the triplet representation of interest and in the Altarelli-Feruglio basis [143], $S$ and $T$ have the form given in Eq. (24).

Assume next that (see, e.g., $[11,14])$

$G_{e}=Z_{3}^{T}=\left\{1, T, T^{2}\right\}, \quad G_{v}=Z_{2}^{S}=\{1, S\}$,

where $Z_{3}^{T}$ and $Z_{2}^{S}$ are two specific $Z_{3}$ and $Z_{2}$ subgroups of $A_{4}$. In this case we have, in particular: $\rho\left(g_{e}\right)=1, T, T^{2}$, $T$ being the diagonal matrix given in Eq. (24). As a consequence, $U_{e}$, which diagonalises $\rho\left(g_{e}\right)=T$, as in the case discussed in the preceding subsection, is just a diagonal phase matrix, whose phases are unphysical, while $M_{e}$ is a diagonal matrix with the masses of the electron, muon and tauon as diagonal elements.

The most general matrix which diagonalises $\rho\left(g_{v}\right)=S$, with $S$ given in Eq. (24) has the form:

$U_{v}^{\circ}=V_{\mathrm{TBM}} U_{13}\left(\theta_{13}^{v}, \alpha\right)$, where $V_{\mathrm{TBM}}$ is the tri-bimaximal (TBM) mixing matrix given in Eq. (26), and

$U_{13}\left(\theta_{13}^{v}, \alpha\right)=\left(\begin{array}{ccc}\cos \theta_{13}^{v} & 0 \sin \theta_{13}^{v} e^{i \alpha} \\ 0 & 1 & 0 \\ -\sin \theta_{13}^{v} e^{-i \alpha} & 0 & \cos \theta_{13}^{v}\end{array}\right)$

The angle $\theta_{13}^{v}$ and the phase $\alpha$ in $U_{13}\left(\theta_{13}^{v}, \alpha\right)$ are arbitrary free parameters. Indeed, it is not difficult to convince oneself that

$S=U_{v}^{\circ} \operatorname{diag}(-1,1,-1)\left(U_{v}^{\circ}\right)^{\dagger}=V_{\mathrm{TBM}} \operatorname{diag}(-1,1,-1) V_{\mathrm{TBM}}^{T}$.

Thus, the matrix $U_{13}\left(\theta_{13}^{v}, \alpha\right)$ appears in the matrix $U_{v}^{\circ}$ diagonalising $S$ as a consequence of the degeneracy of the first and third eigenvalues of $S$.

We see that in the $A_{4}$ model considered, the underlying symmetry form of the PMNS matrix is the tri-bimaximal mixing, $V_{\mathrm{TBM}}$. The matrix $U_{13}\left(\theta_{13}^{\nu}, \alpha\right)$ provides the necessary corrections to $V_{\mathrm{TBM}}$ that lead, in particular, to $\theta_{13} \neq 0$. Thus, the model considered contains two free parameters the angle $\theta_{13}^{v}$ and the phase $\alpha$.

Taking into account the results for the forms of $U_{e}$ and $U_{v}^{\circ}$ we have obtained and Eq. (20), we get the following expression for the PMNS matrix:

$$
\begin{aligned}
U_{\mathrm{PMNS}} & =U_{v}^{\circ} P^{\circ}=V_{\mathrm{TBM}} U_{13}\left(\theta_{13}^{v}, \alpha\right) P^{\circ} \\
& =\left(\begin{array}{ccc}
\sqrt{\frac{2}{3}} c & \frac{1}{\sqrt{3}} & \sqrt{\frac{2}{3}} s e^{i \alpha} \\
-\frac{c}{\sqrt{6}}+\frac{s}{\sqrt{2}} e^{-i \alpha} & \frac{1}{\sqrt{3}} & -\frac{c}{\sqrt{2}}-\frac{s}{\sqrt{6}} e^{i \alpha} \\
-\frac{c}{\sqrt{6}}-\frac{s}{\sqrt{2}} e^{-i \alpha} & \frac{1}{\sqrt{3}} & \frac{c}{\sqrt{2}}-\frac{s}{\sqrt{6}} e^{i \alpha}
\end{array}\right) P^{\circ},
\end{aligned}
$$

where $c \equiv \cos \theta_{13}^{v}$ and $s \equiv \sin \theta_{13}^{v}$.

We will consider next the phenomenological predictions of the discussed $A_{4}$ model of neutrino mixing. Comparing, for example, the absolute values of the elements of the first rows of the PMNS matrix in Eq. (34) and in the standard parametrisation, Eqs. (2) and (3), we get:

$\sin ^{2} \theta_{13}=\frac{2}{3} s^{2}, \quad \sin ^{2} \theta_{12} \cos ^{2} \theta_{13}=\frac{1}{3}$.

Comparing the $U_{\mu 3}$ elements and using the first relation in the preceding equation we find:

$\sin ^{2} \theta_{23}=\frac{1}{c_{13}^{2}}\left|\frac{c}{\sqrt{2}}+\frac{s}{\sqrt{6}} e^{i \alpha}\right|^{2}=\frac{1}{2}+\frac{s_{13}}{2} \frac{\left(2-3 s_{13}^{2}\right)^{\frac{1}{2}}}{\left(1-s_{13}^{2}\right)} \cos \alpha$. 
To leading order in $s_{13}$ we have:

$\frac{1}{2}-\frac{s_{13}}{\sqrt{2}} \lessgtr \sin ^{2} \theta_{23} \lessgtr \frac{1}{2}+\frac{s_{13}}{\sqrt{2}}$, or $0.391 \lessgtr \sin ^{2} \theta_{23} \lessgtr 0.609$,

where the numerical values correspond to the maximal allowed value of $\sin ^{2} \theta_{13}$ at $3 \sigma$ C.L. The interval of possible values of $\sin ^{2} \theta_{23}$ in Eq. (37) lies within the $3 \sigma$ ranges of experimentally allowed values of $\sin ^{2} \theta_{23}$ for $\mathrm{NO}$ and IO spectra, quoted in Table 1.

Further, using the constraint $\left|U_{\mu 2}\right|^{2}=1 / 3$ (or $\left|U_{\tau 2}\right|^{2}=$ $1 / 3$ ) following from the form of $U_{\text {PMNS }}$ in Eq. (34), we obtain the following sum rule for the Dirac phase $\delta$ :

$\cos \delta=\frac{\cos 2 \theta_{23} \cos 2 \theta_{13}}{\sin 2 \theta_{23} \sin \theta_{13}\left(2-3 \sin ^{2} \theta_{13}\right)^{\frac{1}{2}}}$,

where we have expressed $\cos \theta_{12} \sin \theta_{12}$ in terms of $\sin \theta_{13}$ using Eq. (35).

It follows from the preceding brief discussion that $\theta_{13}$ and $\theta_{23}$ of the standard parametrisation of the PMNS matrix are equivalent to the two independent parameters $\theta_{13}^{v}$ and $\alpha$ of the considered $A_{4}$ model, while the angle $\theta_{12}$ and the Dirac phase $\delta$ can be considered as functions of $\theta_{13}$ and $\theta_{23} .{ }^{16}$

The phase $\alpha$ and the Dirac phase $\delta$ are related via

$\sin 2 \theta_{23} \sin \delta=\sin \alpha$

This relation follows from the equality between the expressions of the rephasing invariant $J_{\mathrm{CP}}$, Eq. (6), in the standard parametrisation of the PMNS matrix and in the parametrisation defined in Eq. (34).

As it is not difficult to show, the phase $\alpha$ contributes also to the Majorana phase $\alpha_{31}$ of the PMNS matrix, Eqs. (2) and (3):

$\frac{\alpha_{31}}{2}=\frac{\xi_{31}}{2}+\alpha_{2}+\alpha_{3}$,

where

$\alpha_{2}=\arg \left(-\frac{c}{\sqrt{2}}-\frac{s}{\sqrt{6}} e^{i \alpha}\right), \quad \alpha_{3}=\arg \left(\frac{c}{\sqrt{2}}-\frac{s}{\sqrt{6}} e^{i \alpha}\right)$,

$\sin \alpha_{2}=-\frac{s}{\sqrt{6}} \frac{\sin \alpha}{s_{23} c_{13}}=-\tan \theta_{13} \cos \theta_{23} \sin \delta$,

$\sin \alpha_{3}=-\frac{s}{\sqrt{6}} \frac{\sin \alpha}{c_{23} c_{13}}=-\tan \theta_{13} \sin \theta_{23} \sin \delta$,

$\overline{16}$ Actually, any pair of the four parameters $\theta_{12}, \theta_{23}, \theta_{13}$ and $\delta$ can play the role of the two independent parameters of the model. where we have used Eq. (39). In Eqs. (42) and (43), $\sin \delta$ can be considered as a function of $\theta_{23}$ and $\theta_{13}$ (see Eq. (38)). We also have:

$\sin \left(\alpha-\alpha_{2}-\alpha_{3}\right)=-\sin \delta$.

That the phases $\alpha_{2}$ and $\alpha_{3}$ contribute to the Majorana phase $\alpha_{31}$ can be seen by casting the parametrisation of $U_{\text {PMNS }}$ in Eq. (34) in the standard parametrisation form, Eqs. (2) and (3). This can be done by multiplying the matrix in Eq. (34) on the right by $P_{33}^{*} P_{33}$ with $P_{33}=\operatorname{diag}\left(1,1, e^{i\left(\alpha_{2}+\alpha_{3}\right)}\right)$, and absorbing $P_{33}$ in $P^{\circ}$. The phases $e^{-i \alpha_{3}}$ and $e^{-i \alpha_{2}}$, which after that appear respectively in the $U_{\mu 3}$ and $U_{\tau 3}$ elements of $U_{\text {PMNS }}$ in Eq. (34), are removed from these elements by phase redefinition of the $\mu^{\mp}$ and $\tau^{\mp}$ fields in the weak charged lepton current (1). As a consequence of these simple manipulations the phase factor $e^{i \alpha_{3}}\left(e^{i \alpha_{2}}\right)$ appears in the $U_{\mu 1}$ and $U_{\mu 2}$ ( $U_{\tau 1}$ and $U_{\tau 2}$ ) elements of $U_{\mathrm{PMNS}}$, while the phase factor $e^{i \alpha}$ of the $U_{e 3}$ element (see Eq. (34)) changes to $e^{i\left(\alpha-\alpha_{2}-\alpha_{3}\right)}$. The phases in $P_{33} P^{\circ}$ contribute to the Majorana phases $\alpha_{21} / 2$ and $\alpha_{31} / 2$.

The phenomenology of neutrino mixing described by the PMNS matrix given in (34), apart from the relation (39) and the contribution of the phase $\alpha$ to the Majorana phase $\alpha_{31}$, Eqs. (40)-(43), as well as of the relation (44), was discussed in [144]. The prediction for $\sin ^{2} \theta_{12}$ in Eq. (35) and the sum rule for the Dirac phases $\delta$, Eq. (38), can also be obtained from the general results on neutrino mixing in the case of $A_{4}$ lepton flavour symmetry derived in [14].

Thus, the $A_{4}$ model considered predicts ${ }^{17}$ (i) a correlation between the values of $\sin ^{2} \theta_{12}$ and $\sin ^{2} \theta_{13}: \sin ^{2} \theta_{12}=$ $1 /\left(3\left(1-\sin ^{2} \theta_{13}\right)\right)$, (ii) an interval of possible values of $\sin ^{2} \theta_{23}$, which depends on $\sin \theta_{13}$, and (ii) a sum rule for the Dirac CPV phase $\delta$ by which $\cos \delta$ is expressed in terms of the two measured neutrino mixing angles $\theta_{13}$ and $\theta_{23}$. In this model the Majorana phases $\alpha_{21}$ and $\alpha_{31}$ remain undetermined due to the contribution respectively of the phases $\xi_{21}$ and $\xi_{31}$, which are not fixed.

The correlation between $\sin ^{2} \theta_{12}$ and $\sin ^{2} \theta_{13}$ leads to the prediction $\sin ^{2} \theta_{12}=0.340$, where we have employed the best fit value of $\sin ^{2} \theta_{13}$ in Table 1 . This value lies outside the $2 \sigma$, but is inside the $3 \sigma$, currently allowed intervals of values of $\sin ^{2} \theta_{12}$. Using the best fit values of $\sin ^{2} \theta_{23}$ and $\sin ^{2} \theta_{13}$ for the NO and IO neutrino mass spectrum, given in Table 1, and the sum rule for $\cos \delta$, Eq. (38), we find:

$$
\begin{aligned}
\cos \delta & =0.728(-0.865), \\
\delta & = \pm 43.32^{\circ}\left(180^{\circ} \pm 30.07^{\circ}\right), \quad \mathrm{NO}(\mathrm{IO}) .
\end{aligned}
$$

17 The result for $\sin ^{2} \theta_{12}$ and the sum rule for $\cos \delta$ can be obtained respectively from Eq. (58) in subsection 4.1 and Table 3 (Case B1) in [14] by setting $\sin ^{2} \theta_{12}^{\circ}=1 / 3$ and $\sin ^{2} \theta_{23}^{\circ}=1 / 2$. 
If instead we use the best fit values for $\mathrm{NO}$ (IO) spectrum of $\sin ^{2} \theta_{23}=0.538(0.554)$ and $\sin ^{2} \theta_{13}=0.02206(0.02227)$ reported in [57] we get:

$$
\begin{aligned}
\cos \delta & =-0.353(-500), \\
\delta & =180^{\circ} \pm 69.4^{\circ}\left(180^{\circ} \pm 60.0^{\circ}\right), \quad \mathrm{NO}(\mathrm{IO}) .
\end{aligned}
$$

Thus, as a consequence primarily of the fact that $\cos \delta \propto$ $\cos 2 \theta_{23}$, the predictions for $\cos \delta$, and correspondingly of $\delta$, depend strongly on the values of $\sin ^{2} \theta_{23}$ and can differ significantly for the two neutrino mass orderings. The values of $\delta=43.32^{\circ}, 110.6^{\circ}$ and $120^{\circ}$ are strongly disfavored (if not ruled out) by the current data. It should be added that the difference between the predictions of $\cos \delta(\delta)$ for $\mathrm{NO}$ and IO neutrino mass spectra are due to the difference between the best fit values of $\sin ^{2} \theta_{23}$ for the two spectra (see Table 1 and Eq. (4)). For $\sin ^{2} \theta_{23}=0.5$ we have for both spectra $\cos \delta=$ 0 , or $\delta=\pi / 2,3 \pi / 2$, with $\delta=\pi / 2$ strongly disfavored by the current data.

It follows from the preceding results that the high precision measurement of $\sin ^{2} \theta_{12}$ combined with the data on $\sin ^{2} \theta_{13}$ will allow to critically test the predicted correlation between $\sin ^{2} \theta_{12}$ and $\sin ^{2} \theta_{13}$ of the considered $A_{4}$ model. The high precision measurement of $\sin ^{2} \theta_{23}$, the data on $\sin ^{2} \theta_{13}$ and a sufficiently precise determination of $\delta$ will make it possible to test the sum rule predictions for $\delta$ of the model. With the indicated tests the $A_{4}$ model of neutrino mixing discussed in the present subsection will be either verified or ruled out.

\subsection{Neutrino mixing from $S_{4}$ symmetry}

We will consider next a second rather simple example of generation of neutrino mixing based on the $S_{4}$ symmetry. We recall that the three $S_{4}$ generators $S, T$ and $U$ satisfy the presentation rules given in Eq. (23). In the triplet representation of interest and in the basis employed by us $S, T$ and $U$ are given in Eq. (24).

In this case let us assume that (see, e.g., [14])

$G_{e}=Z_{3}^{T}=\left\{1, T, T^{2}\right\}, \quad G_{v}=Z_{2}^{S U}=\{1, S U\}$,

where $Z_{3}^{T}$, as we have discussed, is a $Z_{3}$ subgroup also of $S_{4}$ and $Z_{2}^{S U}$ is one of the $Z_{2}$ subgroups of $S_{4}$. As in the case of $A_{4}$ symmetry considered in the preceding subsection, $U_{e}$, which diagonalises $\rho\left(g_{e}\right)=T$, is effectively a unit $3 \times 3$ matrix and $M_{e}$ is a diagonal matrix containing the masses of the charged leptons.

The matrix $U_{v}^{\circ}$, which diagonalises the element $\rho\left(g_{v}\right)=$ $S U$ of $Z_{2}^{S U}$ (and $M_{v}^{\dagger} M_{\nu}$ ), with $S$ and $U$ given in Eq. (24), has the following general form:

$$
U_{v}^{\circ}=V_{\mathrm{TBM}} U_{23}\left(\theta_{23}^{v}, \beta\right),
$$

where $V_{\mathrm{TBM}}$ is the TBM mixing matrix and

$$
U_{23}\left(\theta_{23}^{v}, \beta\right)=\left(\begin{array}{ccc}
1 & 0 & 0 \\
0 & \cos \theta_{23}^{v} & \sin \theta_{23}^{v} e^{i \beta} \\
0 & -\sin \theta_{23}^{v} e^{-i \beta} & \cos \theta_{23}^{v}
\end{array}\right),
$$

the angle $\theta_{23}^{v}$ and the phase $\beta$ being arbitrary free parameters. The form of $U_{v}^{\circ}$ follows from the fact that the element $\rho\left(g_{v}\right)=S U$, as is easy to verify, is diagonalised by $V_{\mathrm{TBM}}$. However, in the resulting diagonal matrix the $2 \mathrm{nd}$ and the 3rd eigenvalues are degenerate and thus it is invariant with respect to a unitary transformation with $U_{23}\left(\theta_{23}^{v}, \beta\right)$ :

$$
\begin{gathered}
S U= \pm \\
\qquad V_{\text {TBM }} \operatorname{diag}(-1,1,1) V_{\text {TBM }}^{T}= \pm V_{\text {TBM }} U_{23}\left(\theta_{23}^{v}, \beta\right) \\
\operatorname{diag}(-1,1,1)\left(V_{\text {TBM }} U_{23}\left(\theta_{23}^{v}, \beta\right)\right)^{\dagger} .
\end{gathered}
$$

We see that also in the model with $S_{4}$ symmetry under discussion, the underlying symmetry form of the PMNS matrix is again the TBM one, $V_{\mathrm{TBM}}$. The matrix $U_{23}\left(\theta_{23}^{v}, \beta\right)$ provides the necessary corrections to $V_{\mathrm{TBM}}$ leading, e.g., to $\theta_{13} \neq 0$.

Similarly to the model based on the $A_{4}$ symmetry discussed in the previous subsection, the $S_{4}$ model we are discussing contains two free parameters - the angle $\theta_{23}^{v}$ and the phase $\beta$. However, as we show below, the testable phenomenological predictions of the model with $S_{4}$ symmetry differ significantly from the analogous predictions of the $A_{4}$ model.

From Eqs. (26), (49) and (20) we get for the PMNS matrix:

$$
\begin{aligned}
U_{\mathrm{PMNS}} & =U_{\nu}^{\circ} P^{\circ}=V_{\mathrm{TBM}} U_{23}\left(\theta_{23}^{\nu}, \beta\right) P^{\circ} \\
& =\left(\begin{array}{ccc}
\sqrt{\frac{2}{3}} & \frac{c_{23}^{\nu}}{\sqrt{3}} & \frac{s_{23}^{\nu}}{\sqrt{3}} e^{i \beta} \\
-\frac{1}{\sqrt{6}} & \frac{c_{23}^{\nu}}{\sqrt{3}}+\frac{s_{23}^{\nu}}{\sqrt{2}} e^{-i \beta} & -\frac{c_{23}^{\nu}}{\sqrt{2}}+\frac{s_{23}^{\nu}}{\sqrt{3}} e^{i \beta} \\
-\frac{1}{\sqrt{6}} & \frac{c_{23}^{\nu}}{\sqrt{3}}-\frac{s_{23}^{\nu}}{\sqrt{2}} e^{-i \beta} & \frac{c_{23}^{v}}{\sqrt{2}}+\frac{s_{23}}{\sqrt{3}} e^{i \beta}
\end{array}\right) P^{\circ},
\end{aligned}
$$

where $c_{23}^{v} \equiv \cos \theta_{23}^{v}$ and $s_{23}^{v} \equiv \sin \theta_{23}^{v}$.

Proceeding as in Sect. 3.3 we find:

$\sin ^{2} \theta_{13}=\frac{1}{3} \sin ^{2} \theta_{23}^{v}, \quad \sin ^{2} \theta_{12}=\frac{1}{3} \cos ^{2} \theta_{23}^{v}=\frac{1-3 \sin ^{2} \theta_{13}}{3\left(1-\sin ^{2} \theta_{13}\right)}$.

The neutrino mixing parameter $\sin ^{2} \theta_{23}$ is determined by $\theta_{23}^{v}$ (or $\theta_{13}$ ) and $\beta$ and its value is not predicted:

$$
\begin{aligned}
\sin ^{2} \theta_{23} & =\frac{1}{c_{13}^{2}}\left|-\frac{c_{23}^{\nu}}{\sqrt{2}}+\frac{s_{23}^{\nu}}{\sqrt{3}} e^{i \beta}\right|^{2} \\
& =\frac{1}{2}-\sqrt{2} s_{13} \frac{\left(1-3 s_{13}^{2}\right)^{\frac{1}{2}}}{\left(1-s_{13}^{2}\right)} \cos \beta .
\end{aligned}
$$


To leading order in $s_{13}$ we have:

$$
\begin{aligned}
& \frac{1}{2}-\sqrt{2} s_{13} \lesssim \sin ^{2} \theta_{23} \lesssim \frac{1}{2}+\sqrt{2} s_{13}, \text { or } \\
& 0.293 \lessgtr \sin ^{2} \theta_{23} \lessgtr 0.707,
\end{aligned}
$$

where the numerical values are obtained for the maximal value of $\sin ^{2} \theta_{13}$ allowed at $3 \sigma$ C.L. The interval of values of $\sin ^{2} \theta_{23}$ in Eq. (54) is larger than the $3 \sigma$ experimentally allowed NO and IO intervals of values of $\sin ^{2} \theta_{23}$ (see Table $1)$.

The Dirac phase $\delta$ satisfies the following sum rule:

$$
\begin{aligned}
\cos \delta & =\frac{\frac{1}{6}-c_{23}^{2}+\frac{2}{3 c_{13}^{2}}\left(c_{23}^{2}-s_{23}^{2} s_{13}^{2}\right)}{2 c_{23} s_{23} s_{13} c_{12} s_{12}} \\
& =\frac{\left(-1+5 s_{13}^{2}\right) \cos 2 \theta_{23}}{2 \sqrt{2} \sin 2 \theta_{23} s_{13}\left(1-3 s_{13}^{2}\right)^{\frac{1}{2}}},
\end{aligned}
$$

where we expressed $c_{12} s_{12}$ in terms of $\theta_{13}$ using Eq. (52),

$c_{12} s_{12}=\frac{\sqrt{2}}{3 c_{13}^{2}}\left(1-3 s_{13}^{2}\right)^{\frac{1}{2}}$.

We also have:

$\sin 2 \theta_{23} \sin \delta=\sin \beta$.

Similarly to the phases $\alpha$ of the $A_{4}$ model considered in the preceding subsection, the phase $\beta$ of the discussed $S_{4}$ model contributes to the Majorana phase $\alpha_{31}$ in the standard parametrisation of the PMNS matrix (see Eqs. (2) and (3)):

$\frac{\alpha_{31}}{2}=\frac{\xi_{31}}{2}+\beta_{2}+\beta_{3}$

where

$$
\begin{aligned}
& \beta_{2}=\arg \left(-\frac{c_{23}^{v}}{\sqrt{2}}+\frac{s_{23}^{v}}{\sqrt{3}} e^{i \beta}\right), \quad \beta_{3}=\arg \left(\frac{c_{23}^{\nu}}{\sqrt{2}}+\frac{s_{23}^{v}}{\sqrt{3}} e^{i \beta}\right), \\
& \sin \beta_{2}=\frac{s_{23}^{\nu}}{\sqrt{3}} \frac{\sin \beta}{s_{23} c_{13}}=\tan \theta_{13} \cos \theta_{23} \sin \delta, \\
& \sin \beta_{3}=\frac{s_{23}^{\nu}}{\sqrt{3}} \frac{\sin \beta}{c_{23} c_{13}}=\tan \theta_{13} \sin \theta_{23} \sin \delta,
\end{aligned}
$$

where we have used Eqs. (51) and (57) and $\sin \delta$ in Eqs. (60) and (61) can be considered as a function of $\theta_{23}$ and $\theta_{13}$ (via Eq. (55)). We also have:

$\sin \left(\beta-\beta_{2}-\beta_{3}\right)=-\sin \delta$.

The model with $U_{\mathrm{PMNS}}=V_{\mathrm{TBM}} U_{23}\left(\theta_{23}^{v}, \beta\right)$ was discussed on general phenomenological grounds in [145], where the predictions given in Eqs. (52) and (55) were obtained and the dependence of $\delta$ on $\sin ^{2} \theta_{23}$ for a set of different values of $\theta_{13}$ was studied graphically. The correlation between $\sin ^{2} \theta_{21}$ and $\sin ^{2} \theta_{13}$ and the sum rule for $\cos \delta$ can also can be obtained from the general results for the group $S_{4}$ derived in [14]. ${ }^{18}$

Thus, as in the $A_{4}$ model, $\theta_{13}$ and $\theta_{23}$, or any pair of the four parameters $\theta_{12}, \theta_{23} \theta_{13}$ and $\delta$, can be considered as the two independent parameters of the $S_{4}$ model. The model predicts a correlation between the values of $\sin ^{2} \theta_{12}$ and $\sin ^{2} \theta_{13}$, which for the best fit value of $\sin ^{2} \theta_{13}$ implies $\sin ^{2} \theta_{12}=0.319$. This prediction lies in the current $1 \sigma$ allowed interval of values of $\sin ^{2} \theta_{12}$. Using Eqs. (55), (56) and the best fit values of $\sin ^{2} \theta_{23}$ and $\sin ^{2} \theta_{13}$ from Table 1 , we also get the following predictions for $\cos \delta$ in the cases of NO and IO neutrino mass spectra:

$$
\begin{aligned}
\cos \delta & =-0.338(0.402), \\
\delta & = \pm 109.73^{\circ}\left( \pm 66.27^{\circ}\right), \text { NO }(\mathrm{IO}) .
\end{aligned}
$$

Using instead the best fit values of $\sin ^{2} \theta_{23}$ and $\sin ^{2} \theta_{13}$ for NO (IO) spectrum from [57] we find rather different results due essentially to the difference in the best fit values of $\sin ^{2} \theta_{23}$ :

$$
\begin{aligned}
\cos \delta & =0.167(0.237), \\
\delta & = \pm 80.38^{\circ}\left( \pm 76.30^{\circ}\right), \text { NO (IO). }
\end{aligned}
$$

The values $\delta=109.73^{\circ}, 66.27^{\circ}, 80.38^{\circ}$ and $76.30^{\circ}$ are strongly disfavored by the current data. As in the $A_{4}$ model, the difference between the predictions of $\cos \delta(\delta)$ for $\mathrm{NO}$ and IO neutrino mass spectra are a consequence of the difference between the best fit values of $\sin ^{2} \theta_{23}$ for the two spectra (see Table 1 and Eq. (4)). For $\sin ^{2} \theta_{23}=0.5$ we have for both spectra $\cos \delta=0$, or $\delta=\pi / 2,3 \pi / 2$, also in the $S_{4}$ model, with $\delta=\pi / 2$ strongly disfavored by the current data.

As we have seen, the $A_{4}$ and $S_{4}$ models considered lead to largely different predictions for $\sin ^{2} \theta_{12}$ and, if $\theta_{23} \neq \pi / 4$, for $\cos \delta(\delta)$ as well. These predictions can be used to discriminate experimentally between the two models. In both $A_{4}$ and $S_{4}$ models we have discussed the Majorana phases are not predicted.

\subsection{Comment on the symmetry breaking}

The discrete symmetry approach to neutrino mixing we have discussed so far allows to explain quantitatively the observed pattern of neutrino mixing. A complete selfconsistent (renormalisable) theory based on this approach

\footnotetext{
$\overline{18}$ In [14] a different basis for the $S_{4}$ generators $S, T$ and $U$ has been employed. The results of interest for, e.g., $\sin ^{2} \theta_{12}$ in Eqs. (52) and the sum rule for $\cos \delta$, Eq. (55), follow respectively from Eq. (66) in subsection 4.2 and Table 3 (Case B2) in [14] by setting $\sin ^{2} \theta_{12}^{\circ}=1 / 6$ and $\sin ^{2} \theta_{13}^{\circ}=1 / 5$.
} 
should include also a mechanism of neutrino mass generation as well as details of breaking of the flavour symmetry $G_{f}$ to the residual symmetries $G_{e}$ and $G_{v}$ in the charged lepton and neutrino sectors. As a rule, the non-Abelian flavour symmetry $G_{f}$ is broken spontaneously by a set of scalar fields, flavons, which are singlets with respect to the Standard Theory $S U(2)_{L} \times U(1)_{\mathrm{Y}_{\mathrm{W}}}$ gauge symmetry but transform according certain irreducible representations of $G_{f}$, couple in a $G_{f}$-invariant manner to the LH lepton doublet fields and RH charged lepton $S U(2)_{L}$ singlet fields via Yukawa-type (typically non-renormalisable effective) interactions. These Yukawa-type effective interactions appear in the low-energy limit of a theory which is renormalisable at some high energy scale $\Lambda$ where the symmetry $G_{f}$ is exact (see, e.g., $[9,43,44,136,146])$. The flavons develop non-zero vacuum expectation values in specific directions ("vacuum alignment"). In the case of the $A_{4}$ model considered in Sect. 3.3, for example, the $A_{4}$ symmetry breaking leading to $G_{e}=Z_{3}^{T}$ and $G_{v}=Z_{2}^{S}$ and generating Majorana mass term for the LH flavour neutrino fields can be achieved (i) by assigning $\tilde{e}_{R}(x), \tilde{\mu}_{R}(x)$ and $\tilde{\tau}_{R}(x)$ to the three different singlet representations of $A_{4} \mathbf{1}, \mathbf{1}^{\prime \prime}$ and $\mathbf{1}^{\prime}$ (see Table 2), respectively, (ii) by introducing two $A_{4}$ triplet and two $A_{4}$ singlet flavon scalar fields, which develop vacuum expectation values in specific directions, and (iii) by using the rules of tensor products of irreducible representations for $A_{4}$ (for further details see, e.g., [9-11]).

Discussing the flavon sectors of the models considered is beyond the scope of the present article. Examples of complete self-consistent (renormalisable) models, in which the breaking of the flavour symmetry $G_{f}$ to desired residual symmetries $G_{e}$ and $G_{v}$ with the help of sets of flavon fields developing non-zero vacuum expectation values in requisite directions and, thus, generating $G_{e}$ - invariant charged lepton mass term and $G_{\nu}$ - invariant neutrino Majorana mass term, include, e.g., the models in Refs. [24,25,130,146-149]; for a review see, e.g., Ref. [43].

3.6 Alternative symmetry forms of $U_{v}$ : bimaximal, golden ratio and hexagonal mixing

Thus, TBM can only be an underlying approximate symmetry form of the PMNS neutrino mixing matrix. Other widely discussed underlying (approximate) symmetry forms of the PMNS matrix include: (i) bimaximal (BM) mixing ${ }^{19}$ [151,152], (ii) the golden ratio type A (GRA) mixing $[113,153,154]$, (iii) the golden ratio type $B$ (GRB) mixing $[115,155]$, and iv) hexagonal (HG) mixing $[116,117]$. For all these forms, including the TBM one, the matrix $U_{v}^{\circ}$ has the

\footnotetext{
19 Bimaximal mixing can also be a consequence of the conservation of the lepton charge $L^{\prime}=L_{e}-L_{\mu}-L_{\tau}$ (LC) [150], supplemented by $\mu-\tau$ symmetry.
}

form: $U_{v}^{\circ}=R_{23}\left(\theta_{23}^{v}\right) R_{13}\left(\theta_{13}^{v}\right) R_{12}\left(\theta_{12}^{v}\right)$ with $\theta_{23}^{v}=-\pi / 4$ and $\theta_{13}^{v}=0$ :

$$
\begin{aligned}
U_{v}^{\circ} & =R_{23}\left(\theta_{23}^{v}=-\pi / 4\right) R_{12}\left(\theta_{12}^{v}\right) \\
& =\left(\begin{array}{ccc}
\cos \theta_{12}^{v} & \sin \theta_{12}^{v} & 0 \\
-\frac{\sin \theta_{12}^{v}}{\sqrt{2}} & \frac{\cos \theta_{12}^{v}}{\sqrt{2}} & -\frac{1}{\sqrt{2}} \\
-\frac{\sin \theta_{12}^{v}}{\sqrt{2}} & \frac{\cos \theta_{12}^{v}}{\sqrt{2}} & \frac{1}{\sqrt{2}}
\end{array}\right) .
\end{aligned}
$$

The value of the angle $\theta_{12}^{v}$, and thus of $\sin ^{2} \theta_{12}^{v}$, depends on the symmetry form of $U_{v}^{\circ}$. For the TBM, BM, GRA, GRB and HG forms we have: (i) $\sin ^{2} \theta_{12}^{v}=1 / 3$ (TBM), (ii) $\sin ^{2} \theta_{12}^{v}=$ $1 / 2$ (BM), (iii) $\sin ^{2} \theta_{12}^{v}=(2+\tilde{r})^{-1} \cong 0.276$ (GRA), $\tilde{r}$ being the golden ratio, $\tilde{r}=(1+\sqrt{5}) / 2$, iv) $\sin ^{2} \theta_{12}^{v}=(3-\tilde{r}) / 4 \cong$ 0.345 (GRB), and v) $\sin ^{2} \theta_{12}^{v}=1 / 4$ (HG).

As we have seen in Sects. 3.2 and 3.4, the TBM form of $U_{v}^{\circ}$ can originate from $G_{f}=S_{4}$ symmetry [105-107] (with residual symmetry $G_{v}=Z_{2}^{S} \times Z_{2}^{U}$ ). It can be obtained also from a $G_{f}=A_{4}$ symmetry [103] (with $G_{v}=Z_{2}^{S}$ and the presence of accidental $\mu-\tau$ (i.e., $Z_{2}$ ) symmetry, see, e.g., $[146])){ }^{20}$

The group $G_{f}=S_{4}$ can also be used to generate the BM from of $U_{v}^{\circ}$ (e.g., by choosing $G_{v}=Z_{2}$ combined with an accidental $\mu-\tau$ symmetry) $[14,105-107,136]$.

The GRA form of $U_{v}^{\circ}$ can be obtained from the group $A_{5}$ $[113,114]$, which is the group of even permutations of five objects and is isomorphic to the group of rotational symmetries of the icosahedron. In this case $\sin ^{2} \theta_{12}^{v}=1 /(\tilde{r} \sqrt{5}) \cong$ 0.276 .

The GRB and HG forms of $U_{v}^{\circ}$ can be generated using the groups $G_{f}=D_{10}$ [115] and $G_{f}=D_{12}[116,117]$, respectively. The dihedral groups $D_{10}$ and $D_{12}$ are the groups of symmetries (rotations and reflections) of the regular decagon and dodecagon. ${ }^{21} D_{10}$ and $D_{12}$ lead respectively to $\theta_{12}^{v}=$ $\pi / 5$ (or $\left.\sin ^{2} \theta_{12}^{v}=(3-\tilde{r}) / 4 \cong 0.345\right)$ and $\theta_{12}^{v}=\pi / 6$ (or $\left.\sin ^{2} \theta_{12}^{v}=1 / 4\right)$. The angles $\pi / 5$ and $\pi / 6$ are the external angles of the decagon and dodecagon.

For all the five underlying symmetry forms of $U_{v}^{\circ}$ listed above we have (i) $\theta_{13}^{v}=0$, which should be corrected to the measured value of $\theta_{13} \cong 0.15$, and (ii) $\sin ^{2} \theta_{23}^{v}=0.5$, which

\footnotetext{
${ }^{20}$ The TBM form of $U_{v}^{\circ}$ can also be derived from $G_{f}=T^{\prime}$ - the double covering group of $A_{4}$ (see, e.g., [10]) - with $G_{v}=Z_{2}^{\mathrm{S}}$, provided the left-handed (LH) charged lepton and neutrino fields each transform as triplets of $T^{\prime}$ (see, e.g., [14] for details). Actually, as can be shown [156], when working with 3-dimensional and 1-dimensional representations of $T^{\prime}$, there is no way to distinguish $T^{\prime}$ from $A_{4}$.

21 The groups $D_{10}$ and $D_{12}$, as it is indicated in Table 2, have 1dimensional and 2-dimensional irreducible representations, but do not have 3-dimensional irreducible unitary representations. The problem of how the GRB and HG forms of $U_{v}^{\circ}$ can be generated using the groups $G_{f}=D_{10}$ and $G_{f}=D_{12}$, respectively, is discussed in Refs. [115-117] and we refer the interested reader to these articles.
} 
might also need to be corrected if it is firmly established that $\sin ^{2} \theta_{23}$ deviates significantly from 0.5 . In the case of the BM form $\sin ^{2} \theta_{12}=0.5$, which is ruled out by the existing data and should be corrected. Finally, the value of $\sin ^{2} \theta_{12}^{v}$ for the HG form lies outside the current $2 \sigma$ allowed range of $\sin ^{2} \theta_{12}$ and might need also to be corrected.

The requisite corrections to the discussed underlying symmetry forms of the PMNS matrix can be generated in each specific case by "decreasing" the residual symmetry $G_{v}$ which leads to a given symmetry form. In the case of $G_{f}=S_{4}\left(G_{f}=A_{4}\right)$, as we have seen, this can be achieved by "decreasing" $G_{v}$ from $Z_{2} \times Z_{2}\left(Z_{2}+\right.$ the "accidental" $\mu-\tau$ (i.e., $\left.Z_{2}\right)$ ) symmetry to $Z_{2}$, leading to additional "correcting" matrix factor in $U_{v}^{\circ}$.

As we have mentioned earlier, the corrections can also be provided by the matrix $U_{e}$. This approach was followed in $[12-14,33,39,40,133]$ and corresponds to the case of $G_{f}$ (i) either broken to $G_{e}=Z_{2}$, or (ii) completely broken, by the charged lepton mass term. In this case the PMNS matrix has the following general form [131]:

$U=U_{e}^{\dagger} U_{v}=\left(\tilde{U}_{e}\right)^{\dagger} \Psi U_{v}^{\circ} P^{\circ}$

Here $\tilde{U}_{e}$ is a $3 \times 3$ unitary matrix and $\Psi$ is a diagonal phase matrix. The matrix $\tilde{U}_{e}$ was chosen in $[12,39,40,133]$ to have the following two forms:

A0: $\tilde{U}_{e}=R_{23}^{-1}\left(\theta_{23}^{e}\right) R_{12}^{-1}\left(\theta_{12}^{e}\right) ; \mathbf{B 0}: \tilde{U}_{e}=R_{12}^{-1}\left(\theta_{12}^{e}\right)$.

where $\theta_{12}^{e}$ and $\theta_{23}^{e}$ are free real angle parameters. These two forms appear in a large class of theoretical models of flavour and studies, in which the generation of charged lepton masses is an integral part (see, e.g., [25, 125-127, 130,132]). The phase matrix $\Psi$ in cases $\mathbf{A 0}$ and $\mathbf{B 0}$ is given by $[12,133]$ :

A0 : $\Psi=\operatorname{diag}\left(1, \mathrm{e}^{-i \psi}, \mathrm{e}^{-i \omega}\right)$;

B0 : $\Psi=\operatorname{diag}\left(1, \mathrm{e}^{-i \psi}, 1\right)$.

The phases $\omega$ and/or $\psi$ serve as a source for the Dirac CPV phase $\delta$ of the PMNS matrix and contribute to the Majorana CPV phases of the PMNS matrix $\alpha_{21}$ and $\alpha_{31}$ [12]. We recall that the diagonal phase matrix $P^{\circ}$ in Eq. (66) is given in Eq. (20): it contains two phases, $\xi_{21}$ and $\xi_{31}$, which also contribute to the Majorana phases $\alpha_{21}$ and $\alpha_{31}$, respectively.

\subsection{Predictions for the Dirac CPV Phase}

\subsubsection{The cases of TBM, BM, GRA, GRB and HG symmetry forms corrected by $U_{e}$}

Consider the case of the five underlying symmetry forms of $U_{v}^{\circ}-$ TBM, BM, GRA, GRB and HG - corrected by the matrix $U_{e}$, with the PMNS matrix given in Eq. (66) and the matrices $\tilde{U}_{e}$ and $\Psi$ as given in Eqs. (67) and (68). In this setting the Dirac phase $\delta$ of the PMNS matrix was shown to satisfy the following sum rule [12]:

$$
\begin{aligned}
\cos \delta= & \frac{\tan \theta_{23}}{\sin 2 \theta_{12} \sin \theta_{13}}\left[\cos 2 \theta_{12}^{v}+\left(\sin ^{2} \theta_{12}-\cos ^{2} \theta_{12}^{v}\right)\right. \\
& \left.\times\left(1-\cot ^{2} \theta_{23} \sin ^{2} \theta_{13}\right)\right] .
\end{aligned}
$$

Within the approach employed this sum rule is exact ${ }^{22}$ and is valid for any value of the angle $\theta_{23}^{v}$ [13] (and not only for $\theta_{23}^{v}=-\pi / 4$ of the five discussed symmetry forms of $U_{v}^{\circ}$ ).

As we see, via the sum rule $\cos \delta$ is expressed in terms of the three neutrino mixing angles $\theta_{12}, \theta_{23}, \theta_{13}$ and one fixed (known) parameter $\theta_{12}^{v}$ which depends on the underlying symmetry form (TBM, BM, GRA, GRB, HG) of the PMNS matrix. The difference between the cases A0 and B0 of forms of $\tilde{U}_{e}$ in Eq. (67) is, in particular, in the correlation between the values of $\sin ^{2} \theta_{23}$ and $\sin ^{2} \theta_{13}$ they lead to. In case $\mathbf{A 0}$ of $\tilde{U}_{e}$, the values of $\sin ^{2} \theta_{23}$ and $\sin ^{2} \theta_{13}$ are not correlated and $\sin ^{2} \theta_{23}$ can differ significantly from 0.5 [12]. For the form $\mathbf{B 0}$ of $\tilde{U}_{e}$ we have [12]:

$\sin ^{2} \theta_{23}=\frac{1}{2} \frac{1-2 \sin ^{2} \theta_{13}}{1-\sin ^{2} \theta_{13}} \cong \frac{1}{2}\left(1-\sin ^{2} \theta_{13}\right)$.

Thus, in contrast to the case A0, in case B0 the value of $\sin ^{2} \theta_{23}$ is correlated with the value of $\sin ^{2} \theta_{13}$ and as a consequence $\sin ^{2} \theta_{23}$ can deviate from 0.5 insignificantly - only by $0.5 \sin ^{2} \theta_{13}$.

Qualitatively, the result in Eq. (69) for $\delta$ can be understood as follows. In the parametrisation defined in Eq. (66) with $U_{v}^{\circ}$, $\tilde{U}_{e}$ and $\Psi$ given in (65) and, e.g., by forms B0 in Eqs. (67) and (68), we have:

$U_{\mathrm{PMNS}}=R_{12}\left(\theta_{12}^{e}\right) \Psi R_{23}\left(\theta_{23}^{v}\right) R_{12}\left(\theta_{12}^{v}\right) P^{\circ}$.

The phase $\psi$ in the phase matrix $\Psi$ serves as a source for the Dirac phase $\delta$ (and gives a contribution to the Majorana phases $\alpha_{21,31}$ [12]). It follows from Eq. (71) that in the case under discussion, the three angles $\theta_{12}, \theta_{23}, \theta_{13}$ and the Dirac phase $\delta$ of the standard parametrisation of $U_{\text {PMNS }}$ are expressed in terms of the three parameters $\theta_{12}^{e}, \psi$ and $\theta_{12}^{v}\left(\theta_{23}^{v}=-\pi / 4\right)$. This suggests that it will be possible to express one of the four parameters $\theta_{12}, \theta_{23}, \theta_{13}$ and $\delta$, namely $\delta$, in terms of the other three, hence Eq. (69). Although the case of $\tilde{U}_{e}$ having the form $\mathbf{A 0}$ in Eq. (67) is somewhat more

\footnotetext{
22 The renormalisation group corrections to the sum rule for $\cos \delta$, Eq. (69), in the cases of neutrino Majorana mass term generated by the Weinberg (dimension 5) operator added to (i) the Standard Model, and (ii) the minimal SUSY extension of the Standard Model, have been investigated in $[157,158]$. They were found in [157] to be negligible, e.g., when the Weinberg operator was added to the Standard Model.
} 
complicated, in what concerns $\cos \delta$ one arrives to the same conclusion and result [12].

Given the values of $\sin \theta_{12}, \sin \theta_{23}, \sin \theta_{13}$ and $\theta_{12}^{v}, \cos \delta$ is determined uniquely by the sum rule (69). This allows us to determine also $|\sin \delta|$ uniquely. However, in absence of additional information, $\operatorname{sgn}(\sin \delta)$ remains undetermined, which leads to a two-fold ambiguity in the determination of the value of $\delta$ from the given value of $\cos \delta$.

The fact that the value of the Dirac CPV phase $\delta$ is determined (up to an ambiguity of the sign of $\sin \delta$ ) by the values of the three mixing angles $\theta_{12}, \theta_{23}$ and $\theta_{13}$ of the PMNS matrix and the value of $\theta_{12}^{v}$ of the matrix $U_{v}^{\circ}$, Eq. (65), is the most striking prediction of the models considered. This result implies that in the schemes under discussion, the rephasing invariant $J_{\mathrm{CP}}$ associated with the Dirac phase $\delta$, Eq. (6), is also a function of the three angles $\theta_{12}, \theta_{23}$ and $\theta_{13}$ of the PMNS matrix and of $\theta_{12}^{v}$ :

$$
\begin{aligned}
J_{\mathrm{CP}} & =J_{\mathrm{CP}}\left(\theta_{12}, \theta_{23}, \theta_{13}, \delta\left(\theta_{12}, \theta_{23}, \theta_{13}, \theta_{12}^{v}\right)\right) \\
& =J_{\mathrm{CP}}\left(\theta_{12}, \theta_{23}, \theta_{13}, \theta_{12}^{v}\right) .
\end{aligned}
$$

This allows to obtain predictions for the values of $J_{\mathrm{CP}}$ for the different symmetry forms of $\tilde{U}_{v}$ (specified by the value of $\theta_{12}^{v}$ ) using the current data on $\theta_{12}, \theta_{23}$ and $\theta_{13}$.

In [12], by using the sum rule in Eq. (69), predictions for $\cos \delta, \delta$ and the $J_{\mathrm{CP}}$ factor were obtained in the TBM, BM, GRA, GRB and HG cases for the b.f.v. of $\sin ^{2} \theta_{12}$, $\sin ^{2} \theta_{23}$ and $\sin ^{2} \theta_{13}$. It was found that the predictions of $\cos \delta$ vary significantly with the symmetry form of $\tilde{U}_{v}$. For the b.f.v. of $\sin ^{2} \theta_{12}=0.308, \sin ^{2} \theta_{13}=0.0234$ and $\sin ^{2} \theta_{23}=0.437$ found for NO spectrum in [56], for instance, one gets [12] $\cos \delta=(-0.0906),(-1.16)$, 0.275, (-0.169) and 0.445, for the TBM, BM (LC), GRA, GRB and $\mathrm{HG}$ forms, respectively. For the TBM, GRA, $\mathrm{GRB}$ and $\mathrm{HG}$ forms these values correspond to $\delta=$ $\pm 95.2^{\circ}, \pm 74.0^{\circ}, \pm 99.7^{\circ}, \pm 63.6^{\circ}$. For the b.f.v. given in Table 1 and obtained in the global analysis [54] one finds in the cases of the TBM, BM (LC), GRA, GRB and HG forms the values given in Table 3. Due to the different NO and IO b.f.v. of $\sin ^{2} \theta_{23}$, the predicted values of $\cos \delta$ and $\delta$ for IO spectrum differ (in certain cases significantly) from those for the NO spectrum.

Three comments are in order. First, according to the results found in [54] and quoted in Table 1, the predicted values of $\delta$ lying in the first quadrant are strongly disfavored (if not ruled out) by the current data. Second, the unphysical value of $\cos \delta$ in the $\mathrm{BM}$ (LC) case is a reflection of the fact that the scheme under discussion with BM (LC) form of the matrix $U_{v}^{\circ}$ does not provide a good description of the current data on $\theta_{12}, \theta_{23}$ and $\theta_{13}$ [133]. Physical values of $\cos \delta$ can be obtained in the case of the NO spectrum, e.g., for the b.f.v. of $\sin ^{2} \theta_{13}$ if the value of $\sin ^{2} \theta_{12}\left(\sin ^{2} \theta_{23}\right)$ is larger (smaller) than the current best fit value ${ }^{23}[12,39,40]$. However, with the b.f.v. of $\sin ^{2} \theta_{23}$ quoted in Eq. (4), the BM (LC) form is strongly disfavored for both NO and IO spectra.

Third, the $A_{4}$ and $S_{4}$ models considered Sects. 3.3 and 3.4 lead to largely different predictions for $\sin ^{2} \theta_{12}$ and, if $\theta_{23} \neq \pi / 4$, for $\cos \delta$ as well, which differ also from the predictions for $\cos \delta$ we have obtained in the cases of the five different symmetry forms - TBM, BM, GRA, GRB, HG and the matrix $\tilde{U}_{e}$ given by the forms $\mathbf{A 0}$ and $\mathbf{B 0}$ in Eq. (67). These predictions can be used to discriminate experimentally between the different models.

The results quoted above imply [12] that a measurement of $\cos \delta$ can allow to distinguish between at least some of the different symmetry forms of $U_{v}^{\circ}$ provided $\theta_{12}, \theta_{13}$ and $\theta_{23}$ are known, and $\cos \delta$ is measured, with sufficiently high precision. ${ }^{24}$ Even determining the sign of $\cos \delta$ will be sufficient to eliminate some of the possible symmetry forms of $\tilde{U}_{v}$.

These conclusions were confirmed by the statistical analyses performed in Refs. [39,40] where predictions of the sum rule (69) for (i) $\delta, \cos \delta$ and the rephasing invariant $J_{\mathrm{CP}}$ using the "data" (best fit values and $\chi^{2}$-distributions) on $\sin ^{2} \theta_{12}$, $\sin ^{2} \theta_{13}, \sin ^{2} \theta_{23}$ and $\delta$ from [56], and (ii) for $\cos \delta$, using prospective uncertainties on $\sin ^{2} \theta_{12}, \sin ^{2} \theta_{13}$ and $\sin ^{2} \theta_{23}$, were derived for the TBM, BM (LC), GRA, GRB and HG symmetry forms of the matrix $\tilde{U}_{v}$. Both analyses were performed for the case of NO neutrino mass spectrum. The results for the IO spectrum are similar. The aim of the first analysis, the results of which for $J_{\mathrm{CP}}$ are shown in Fig. 1 and are summarised in Table 4, was to derive the allowed ranges for $\delta$ and $J_{\mathrm{CP}}$, predicted on the basis of the current data on the neutrino mixing parameters for each of the symmetry forms of $U_{v}^{\circ}$ considered (see [39,40] for details of the analysis). It was found in $[39,40]$, in particular, that the CP-conserving value of $J_{\mathrm{CP}}=0$ is excluded in the cases of the TBM, GRA, GRB and HG neutrino mixing symmetry forms, respectively, at approximately $5 \sigma, 4 \sigma, 4 \sigma$ and $3 \sigma$ C.L. with respect to the C.L. of the corresponding best fit values which all lie in the interval $J_{\mathrm{CP}}=(-0.034)-(-0.031)$ (see Table 4$)$. The best fit value for the BM (LC) form is much smaller and close to zero: $J_{\mathrm{CP}}=\left(-5 \times 10^{-3}\right)$. For the TBM, GRA, GRB and HG forms at $3 \sigma$ we have $0.020 \leq\left|J_{\mathrm{CP}}\right| \leq 0.039$. Thus, for these four forms the $\mathrm{CP}$ violating effects in neutrino oscillations are predicted to be relatively large and observable in the T2HK and DUNE experiments [89,101]. These conclusions hold if one uses in the analysis the results on the neutrino

\footnotetext{
${ }^{23}$ For, e.g., $\sin ^{2} \theta_{12}=0.34$ allowed at $2 \sigma$ by the current data, we have $\cos \delta=-0.943$. Similarly, for $\sin ^{2} \theta_{12}=0.32, \sin ^{2} \theta_{23}=0.41$ and $\sin \theta_{13}=0.158$ we have [12]: $\cos \delta=-0.978$.

${ }^{24}$ Detailed results on the dependence of the predictions for $\cos \delta$ on $\sin ^{2} \theta_{12}, \sin ^{2} \theta_{23}$ and $\sin ^{2} \theta_{13}$ when the latter are varied in their respective $3 \sigma$ experimentally allowed ranges can be found in $[39,40]$.
} 
Table 3 Predicted values of $\cos \delta$ and $\delta$ for the five symmetry forms, TBM, BM, GRA, GRB and $\mathrm{HG}$, and $\tilde{U}_{e}$ given by the form $\mathrm{A0}$ in Eq. (67), obtained using Eq. (69) and the best fit values of $\sin ^{2} \theta_{12}, \sin ^{2} \theta_{23}$ and $\sin ^{2} \theta_{13}$ for NO and IO neutrino mass spectra from Ref. [54] (From Refs. [39,41].)

\begin{tabular}{lclcl}
\hline Scheme & $\cos \delta(\mathrm{NO})$ & $\delta(\mathrm{NO})$ & $\cos \delta(\mathrm{IO})$ & $\delta(\mathrm{IO})$ \\
\hline TBM & -0.16 & $\pm 99^{\circ}$ & -0.27 & $\pm 106^{\circ}$ \\
BM $(\mathrm{LC})$ & -1.26 & $\cos \delta$-unphysical & -1.78 & $\cos \delta$-unphysical \\
GRA & 0.21 & $\pm 78^{\circ}$ & 0.24 & $\pm 76^{\circ}$ \\
GRB & -0.24 & $\pm 105^{\circ}$ & -0.38 & $\pm 112^{\circ}$ \\
HG & 0.39 & $\pm 67^{\circ}$ & 0.48 & $\pm 62^{\circ}$ \\
\hline
\end{tabular}
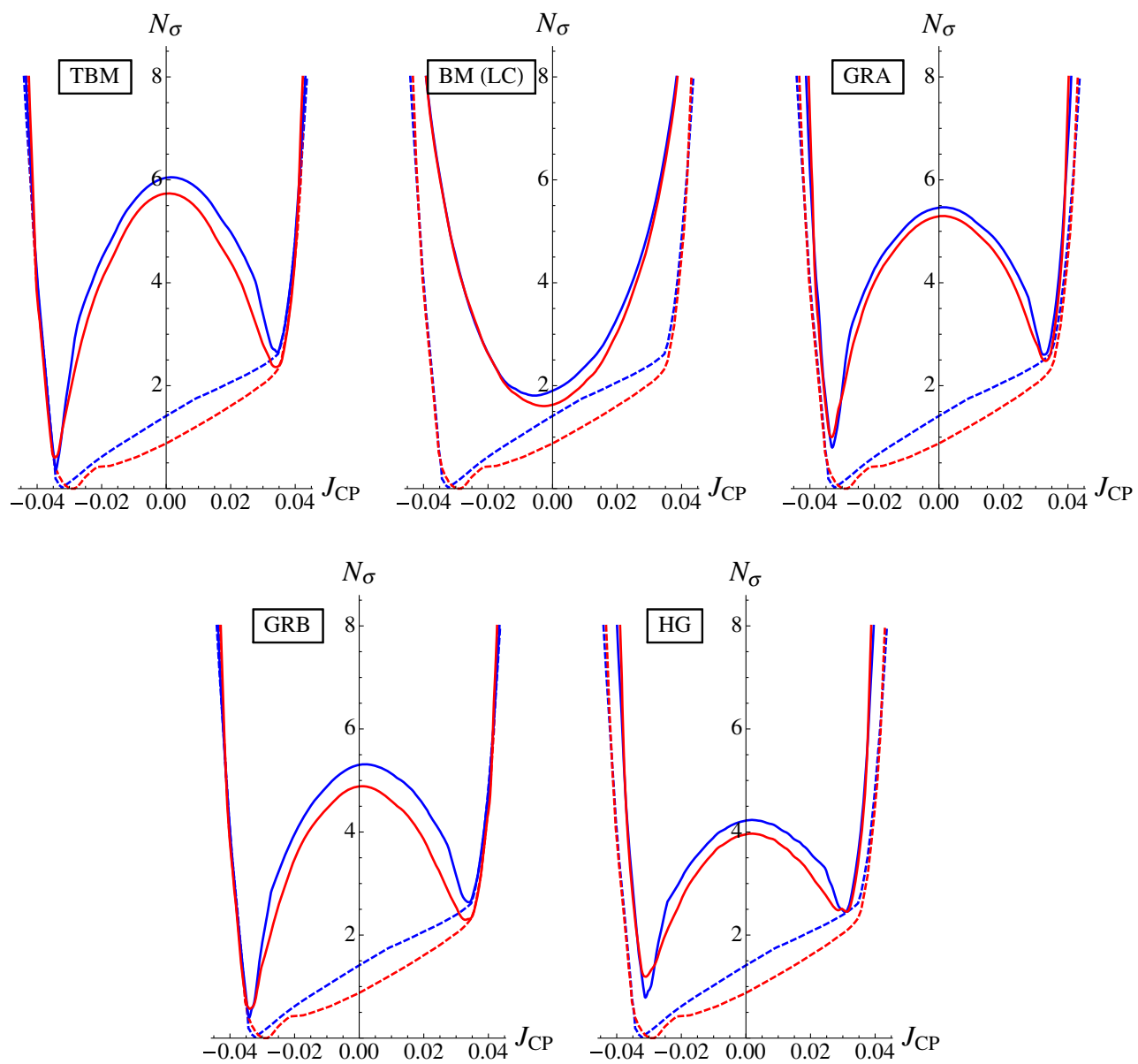

Fig. $1 N_{\sigma} \equiv \sqrt{\chi^{2}}$ as a function of $J_{\mathrm{CP}}$. The dashed lines represent the results of the global fit [56], while the solid lines represent the results we obtain for the TBM, BM (LC), GRA (upper left, central, right panels),

mixing parameters and $\delta$, obtained in the more recent global analyses $[54,57,58]$.

In Fig. 2 (left panel) we present the results of the statistical analysis of the predictions for $\cos \delta$, namely the likelihood function versus $\cos \delta$ within the Gaussian approximation (see $[39,40]$ for details) performed using the b.f.v. of the mixing angles for NO neutrino mass spectrum given in Ref. [56] and the prospective rather small $1 \sigma$ uncertainties (i) of $0.7 \%$ on $\sin ^{2} \theta_{12}$, planned to be reached in JUNO experiment [86], (ii) of $3 \%$ on $\sin ^{2} \theta_{13}$, foreseen to be obtained in the Daya Bay experiment $[159,160]$, and (iii) of $5 \%$ on $\sin ^{2} \theta_{23}$, expected to
GRB and HG (lower left and right panels) neutrino mixing symmetry forms. The blue (red) lines are for NO (IO) neutrino mass spectrum. (From Ref. [39].)

be reached in the currently running and future planned long baseline neutrino oscillation experiments. In the proposed upgrading of the currently taking data T2K experiment [100], for example, $\theta_{23}$ is estimated to be determined with a $1 \sigma$ error of $1.7^{\circ}, 0.5^{\circ}$ and $0.7^{\circ}$ if the best fit value of $\sin ^{2} \theta_{23}=0.50$, 0.43 and 0.60 , respectively. This implies that for these three values of $\sin ^{2} \theta_{23}$ the absolute (relative) $1 \sigma$ error would be 0.0297 (5.94\%), 0.0086 (2\%) and 0.0120 (2\%). This error on $\sin ^{2} \theta_{23}$ is expected to be further reduced in the future planned T2HK [101] and DUNE [89] experiments. 
Table 4 Best fit values of $J_{\mathrm{CP}}$ and $\cos \delta$ and corresponding $3 \sigma$ ranges (found fixing $\left.\chi^{2}-\chi_{\min }^{2}=9\right)$ for the five symmetry forms, TBM, BM, GRA, GRB and $\mathrm{HG}$, and $\tilde{U}_{e}$ given by the form $\mathbf{A 0}$ in Eq. (67) obtained using the data from [56] for NO neutrino mass spectrum. (From Refs. [39,40], where results for IO spectrum are also given.)

\begin{tabular}{|c|c|c|c|c|}
\hline Scheme & $J_{\mathrm{CP}} / 10^{-2}$ (b.f.v.) & $J_{\mathrm{CP}} / 10^{-2}(3 \sigma$ range $)$ & $\cos \delta$ (b.f.v.) & $\cos \delta(3 \sigma$ range $)$ \\
\hline TBM & -3.4 & {$[-3.8,-2.8] \cup[3.1,3.6]$} & -0.07 & {$[-0.47,0.21]$} \\
\hline $\mathrm{BM}(\mathrm{LC})$ & -0.5 & {$[-2.6,2.1]$} & -0.99 & {$[-1.00,[-0.72]$} \\
\hline GRA & -3.3 & {$[-3.7,-2.7] \cup[3.0,3.5]$} & 0.25 & {$[-0.08,0.69]$} \\
\hline GRB & -3.4 & {$[-3.9,-2.6] \cup[3.1,3.6]$} & -0.15 & {$[-0.57,0.13]$} \\
\hline HG & -3.1 & {$[-3.5,-2.0] \cup[2.6,3.4]$} & 0.47 & {$[0.16,0.80]$} \\
\hline
\end{tabular}

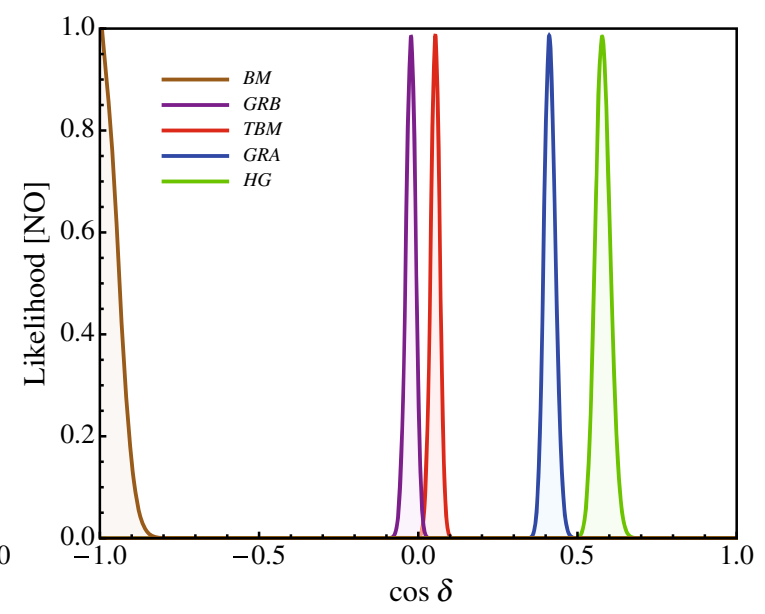

sian approximation. In the left (right) panel $\sin ^{2} \theta_{12}$ is set to its b.f.v. of [56] 0.308 (is set to 0.332), the other mixing angles being fixed to their NO best fit values taken from [56]. See text for further details. (From Ref. [39].)

T2HK, T2HKK (ESSvSB) [101,102,161]. Therefore a measurement of $\cos \delta$ in the quoted range with $\Delta(\cos \delta)=0.08$ will allow one to distinguish between the TBM/GRB, BM (LC) and GRA/HG forms at approximately $3 \sigma$ C.L., if the precision achieved on $\sin ^{2} \theta_{12}, \sin ^{2} \theta_{13}$ and $\sin ^{2} \theta_{23}$ is the same as in Fig. 2.

A more detailed study of the possibility to distinguish between BM (LC), TBM, GRB, GRA and HG forms of $U_{v}^{\circ}$ using the prospective data from DUNE and T2HK experiments was performed in [41]. Some of the results of this study are illustrated in Fig. 3. As is shown in [41] and is indicated by Fig. 3, the combined analysis of the data from the DUNE and T2HK experiments would allow to distinguish between TBM and HG (GRA) symmetry forms of the PMNS matrix at approximately $3 \sigma(2 \sigma)$ confidence level; and the same data would allow to distinguish between GRB and HG (GRA) forms at more than $3 \sigma$ (at approximately $2 \sigma$ ) confidence level. Using the data from the T2HK, T2HKK and DUNE experiments is expected to lead to a better discrimination between the different symmetry forms of $U_{\text {PMNS }}$ owing
${ }^{25}$ Self-consistent models or theories of (lepton) flavour which lead to the GRB form of $U_{v}^{\circ}$ might still be possible to distinguish from those leading to the TBM form using the specific predictions of the two types of models for the neutrino mixing angles. The same observation applies to models which lead to the GRA and HG forms of $U_{v}^{\circ}$. 
Fig. 3 Sensitivities of the experiments DUNE, T2HK and their combined (prospective) data to the symmetry form parameter $\sin ^{2} \theta_{12}^{v}$ allowing to distinguish between the TBM, GRA, GRB, and HG symmetry forms under the assumption that one of them is realised in Nature. In the top left and right panels the assumed true symmetry forms are respectively TBM $\left(\sin ^{2} \theta_{12}^{v}=1 / 3\right)$ and GRA $\left(\sin ^{2} \theta_{12}^{v}=0.276\right)$, while in the bottom left and right panel these forms are GRB

$\left(\sin ^{2} \theta_{12}^{\nu}=0.345\right)$ and $\mathrm{HG}$ $\left(\sin ^{2} \theta_{12}^{v}=0.25\right)$. See text for further details. (From Ref. [41].)
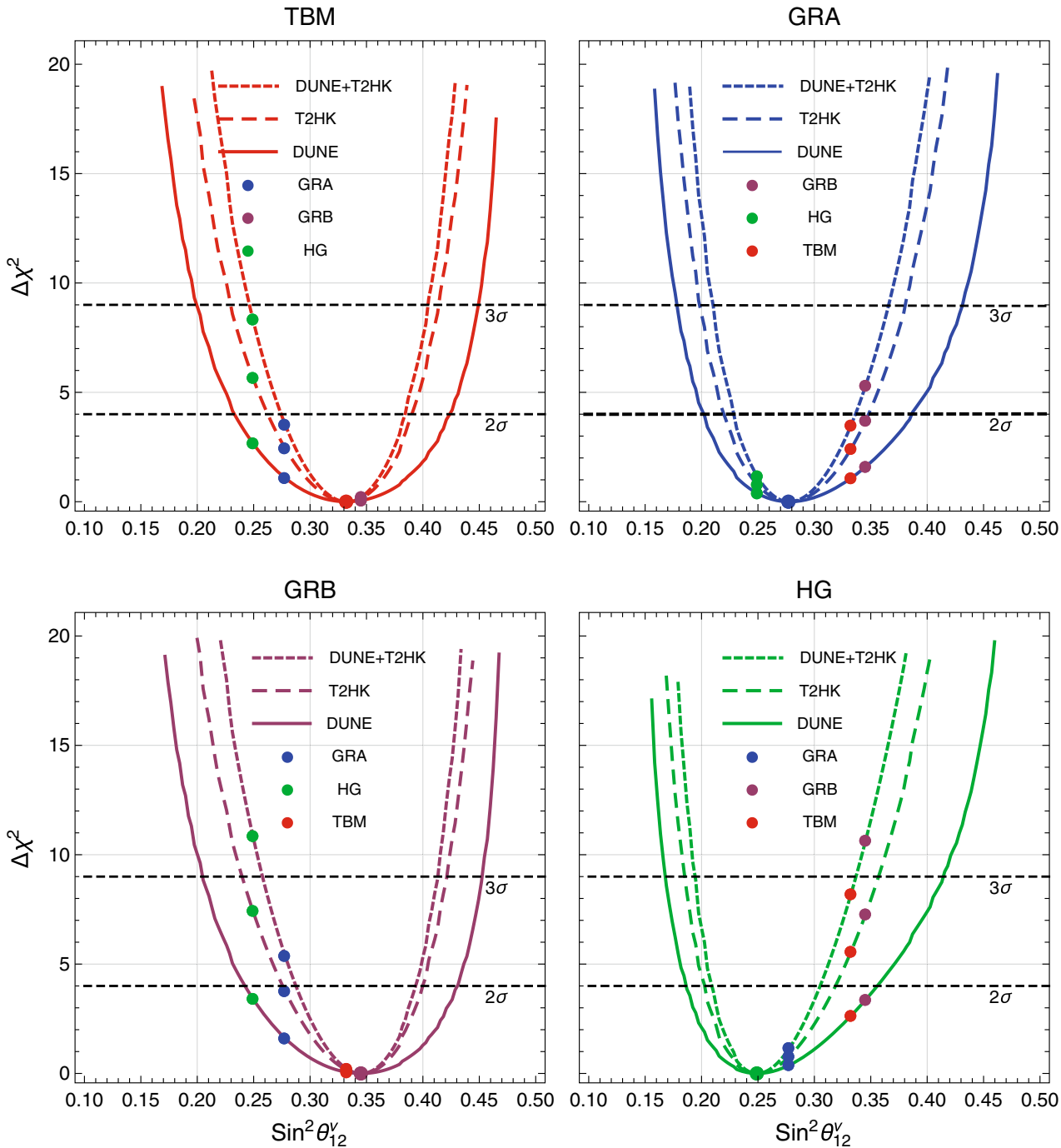

to the better prospective sensitivity to $\delta$ of the combined data from the T2HK and T2HKK experiments.

In what concerns the BM (LC) form, as we have already discussed, it is not compatible with the best fit values of the neutrino mixing angles (leading to $|\cos \delta|>1$ ), but is viable if the $2 \sigma$ ranges of the neutrino mixing angles are taken into account. If, e.g., one keeps $\sin ^{2} \theta_{13}$ and $\sin ^{2} \theta_{23}$ fixed at their best fit values for NO spectrum, one finds $\cos \delta=-1$, and thus a viable BM (LC) mixing form, for $\sin ^{2} \theta_{12}=0.334$, which is the upper limit of the allowed $2 \sigma$ range of $\sin ^{2} \theta_{12}$ (see Table 1). For the indicated choice of values of $\sin ^{2} \theta_{13}$, $\sin ^{2} \theta_{23}$ and $\sin ^{2} \theta_{12}$ the BM form, as was shown in [41], can be distinguished from the other four symmetry forms - TBM, GRB, GRA and $\mathrm{HG}-$ at more than $5 \sigma$ using only the data from the DUNE experiment.

\subsubsection{Alternative Cases and the Power of Data}

In [13] the analyses performed in $[12,39,40]$ was extended by obtaining sum rules for $\cos \delta$ for $U_{\text {PMNS }}$ having the general form given in Eq. (66) and the following forms of $\tilde{U}_{e}$ and $U_{v}^{\circ 26}$ :

C0. $U_{v}^{\circ}=R_{23}\left(\theta_{23}^{v}\right) R_{12}\left(\theta_{12}^{v}\right)$ with $\theta_{23}^{v}=-\pi / 4$ and $\theta_{12}^{v}$ as dictated by TBM, BM, GRA, GRB or HG mixing, and (i) $\tilde{U}_{e}=R_{13}^{-1}\left(\theta_{13}^{e}\right)\left(\Psi=\operatorname{diag}\left(1,1, e^{-i \omega}\right)\right)$, (ii) $\tilde{U}_{e}=R_{23}^{-1}\left(\theta_{23}^{e}\right) R_{13}^{-1}\left(\theta_{13}^{e}\right)\left(\Psi=\operatorname{diag}\left(1, e^{-i \psi}, e^{-i \omega}\right)\right)$, and (iii) $\tilde{U}_{e}=R_{13}^{-1}\left(\theta_{13}^{e}\right) R_{12}^{-1}\left(\theta_{12}^{e}\right)\left(\Psi=\operatorname{diag}\left(1, e^{-i \psi}\right.\right.$, $\left.\left.e^{-i \omega}\right)\right)$;

D0. $U_{v}^{\circ}=R_{23}\left(\theta_{23}^{v}\right) R_{13}\left(\theta_{13}^{v}\right) R_{12}\left(\theta_{12}^{v}\right)$ with $\theta_{23}^{v}, \theta_{13}^{v}$ and $\theta_{12}^{v}$ fixed by arguments associated with symmetries, and iv) $\tilde{U}_{e}=R_{12}^{-1}\left(\theta_{12}^{e}\right)\left(\Psi=\operatorname{diag}\left(1, e^{-i \psi}, 1\right)\right)$, and v) $\tilde{U}_{e}=$ $R_{13}^{-1}\left(\theta_{13}^{e}\right)\left(\Psi=\operatorname{diag}\left(1,1, e^{-i \omega}\right)\right)$.

${ }^{26}$ In [13] a systematic analysis of the forms of $\tilde{U}_{e}$ and $U_{v}^{\circ}$, for which sum rules for $\cos \delta$ of the type of Eq. (69) could be derived, but did not exist in the literature, was performed. 
The sum rules for $\cos \delta$ were derived first for $\theta_{23}^{v}=-\pi / 4$ for the cases listed in point $\mathbf{C} \mathbf{0}$, and for the specific values of (some of) the angles in $U_{v}^{\circ}$, characterising the cases listed in point D0, as well as for arbitrary fixed values of all angles contained in $U_{v}^{\circ}$. In certain models with $\sin ^{2} \theta_{13}^{v} \neq 0, \sin ^{2} \theta_{23}$ is predicted to have specific values which differ significantly from those in case $\mathbf{B 0}$ [13]: $\sin ^{2} \theta_{23}=0.455$; or 0.463 ; or 0.537 ; or 0.545 , the uncertainties in these predictions being insignificant.

Predictions for correlations between neutrino mixing angle values and/or sum rules for $\cos \delta$, which can be tested experimentally, were further derived in [14] for a large number of models based on $G_{f}=S_{4}, A_{4}, T^{\prime}$ and $A_{5}$ and all symmetry breaking patterns, i.e., all possible combinations of residual symmetries, which could lead to the correlations and sum rules of interest: rules can exist also in the case of pattern $\mathbf{D}(\mathbf{E})$ if due to additional assumptions (e.g., additional symmetries) the otherwise unconstrained unitary matrix $U_{e}\left(U_{v}\right)$ is constrained to have the specific form of a matrix of $U(2)$ transformation in a plane or of the product of two $U(2)$ transformations in two different planes [12-14,39,40,133]. Thus, the cases of patterns $\mathbf{D}$ and $\mathbf{E}$ leading to interesting phenomenological predictions are "non-minimal" from the point of view of the symmetries employed (see, e.g., [25,125-128,130]), compared to patterns $\mathbf{A}, \mathbf{B}$ and $\mathbf{C}$ characterised by non-trivial residual symmetries present in both charged lepton and neutrino sectors, which originate from just one non-Abelian flavour symmetry.

As was shown in [14], in the case of pattern $\mathbf{A}, U_{v}^{\circ}$ is fixed by $G_{\nu}$. There are three different general sub-cases, A1, A2 and $\mathrm{A} 3$, corresponding to $U_{e}$ determined up to a unitary rotation in the $1-2,1-3$ and 2-3 plane, respectively. In subcases $\mathrm{A} 1$ and $\mathrm{A} 2$ one obtains a correlation between $\sin ^{2} \theta_{23}$ and $\sin ^{2} \theta_{13}$ and a sum rule for $\cos \delta$, while in sub-case $\mathrm{A} 3$, $\sin ^{2} \theta_{13}$ and $\sin ^{2} \theta_{12}$ are predicted and $\delta$ is not constrained:

$$
\begin{aligned}
& \sin ^{2} \theta_{23}=1-\frac{\cos ^{2} \theta_{13}^{\circ} \cos ^{2} \theta_{23}^{\circ}}{1-\sin ^{2} \theta_{13}}, \quad \mathrm{~A} 1, \\
& \cos \delta=\frac{\cos ^{2} \theta_{13}\left(\sin ^{2} \theta_{23}^{\circ}-\cos ^{2} \theta_{12}\right)+\cos ^{2} \theta_{13}^{\circ} \cos ^{2} \theta_{23}^{\circ}\left(\cos ^{2} \theta_{12}-\sin ^{2} \theta_{12} \sin ^{2} \theta_{13}\right)}{\sin 2 \theta_{12} \sin \theta_{13}\left|\cos \theta_{13}^{\circ} \cos \theta_{23}^{\circ}\right|\left(\cos ^{2} \theta_{13}-\cos ^{2} \theta_{13}^{\circ} \cos ^{2} \theta_{23}^{\circ}\right)^{\frac{1}{2}}}, \mathrm{~A} 1, \\
& \sin ^{2} \theta_{23}=\frac{\sin ^{2} \theta_{23}^{\circ}}{1-\sin ^{2} \theta_{13}}, \quad \mathrm{~A} 2, \\
& \cos \delta=-\frac{\cos ^{2} \theta_{13}\left(\cos ^{2} \theta_{12}^{\circ} \cos ^{2} \theta_{23}^{\circ}-\cos ^{2} \theta_{12}\right)+\sin ^{2} \theta_{23}^{\circ}\left(\cos ^{2} \theta_{12}-\sin ^{2} \theta_{12} \sin ^{2} \theta_{13}\right)}{\sin 2 \theta_{12} \sin \theta_{13}\left|\sin \theta_{23}^{\circ}\right|\left(\cos ^{2} \theta_{13}-\sin ^{2} \theta_{23}^{\circ}\right)^{\frac{1}{2}}}, \mathrm{~A} 2, \\
& \sin ^{2} \theta_{13}=\sin ^{2} \theta_{13}^{\circ}, \sin ^{2} \theta_{12}=\sin ^{2} \theta_{12}^{\circ}, \cos \delta-\text { unconstrained, A3, }
\end{aligned}
$$

(A) $G_{e}=Z_{2}$ and $G_{v}=Z_{n}, n>2$ or $Z_{n} \times Z_{m}, n, m \geq 2$;

(B) $G_{e}=Z_{n}, n>2$ or $G_{e}=Z_{n} \times Z_{m}, n, m \geq 2$ and $G_{v}=Z_{2}$

(C) $G_{e}=Z_{2}$ and $G_{v}=Z_{2}$;

(D) $G_{e}$ is fully broken and $G_{v}=Z_{n}, n>2$ or $Z_{n} \times Z_{m}$, $n, m \geq 2$

(E) $G_{e}=Z_{n}, n>2$ or $Z_{n} \times Z_{m}, n, m \geq 2$ and $G_{v}$ is fully broken.

The three LH neutrino fields and the three LH charged lepton fields were assumed in [14] to transform under the action of $G_{f}$ by a 3-dimensional irreducible representation of $G_{f}$. In this case, as we have already remarked, the results obtained for $A_{4}$ and $T^{\prime}$ coincide. For each pattern, sum rules, i.e., relations between the neutrino mixing angles and/or between the neutrino mixing angles and the Dirac CPV phase $\delta$, when present, were derived. We note that neutrino mixing sum where the angles $\theta_{13}^{\circ}, \theta_{23}^{\circ}$ and $\theta_{12}^{\circ}$ are fixed once the flavour symmetry group $G_{f}$ and the residual symmetry subgroups $G_{e}$ and $G_{v}$ are specified.

In the case of pattern $\mathbf{B}$, of which there are also of three different sub-cases, B1, B2 and B3, corresponding to $U_{e}$ fixed by $G_{e}$ and $U_{\nu}^{\circ}$ determined up to a unitary rotation in the 1$3,2-3$ and 1-2 plane, respectively, there exist a correlation between $\sin ^{2} \theta_{12}$ and $\sin ^{2} \theta_{13}$ and a sum rule for $\cos \delta$ (subcases B1, B2), or $\sin ^{2} \theta_{13}$ and $\sin ^{2} \theta_{23}$ are predicted while $\delta$ remains unconstrained (sub-case $\mathrm{B} 3$ ): 


$$
\begin{aligned}
& \sin ^{2} \theta_{12}=\frac{\sin ^{2} \theta_{12}^{\circ}}{1-\sin ^{2} \theta_{13}}, \quad \mathrm{~B} 1, \\
& \cos \delta=-\frac{\cos ^{2} \theta_{13}\left(\cos ^{2} \theta_{12}^{\circ} \cos ^{2} \theta_{23}^{\circ}-\cos ^{2} \theta_{23}\right)+\sin ^{2} \theta_{12}^{\circ}\left(\cos ^{2} \theta_{23}-\sin ^{2} \theta_{13} \sin ^{2} \theta_{23}\right)}{\sin 2 \theta_{23} \sin \theta_{13}\left|\sin \theta_{12}^{\circ}\right|\left(\cos ^{2} \theta_{13}-\sin ^{2} \theta_{12}^{\circ}\right)^{\frac{1}{2}}}, \mathrm{~B} 1, \\
& \sin ^{2} \theta_{12}=1-\frac{\cos ^{2} \theta_{12}^{\circ} \cos ^{2} \theta_{13}^{\circ}}{1-\sin ^{2} \theta_{13}}, \quad \mathrm{~B} 2, \\
& \cos \delta=\frac{\cos ^{2} \theta_{13}\left(\sin ^{2} \theta_{12}^{\circ}-\cos ^{2} \theta_{23}\right)+\cos ^{2} \theta_{12}^{\circ} \cos ^{2} \theta_{13}^{\circ}\left(\cos ^{2} \theta_{23}-\sin ^{2} \theta_{13} \sin ^{2} \theta_{23}\right)}{\sin 2 \theta_{23} \sin \theta_{13}\left|\cos \theta_{12}^{\circ} \cos \theta_{13}^{\circ}\right|\left(\cos ^{2} \theta_{13}-\cos ^{2} \theta_{12}^{\circ} \cos ^{2} \theta_{13}^{\circ}\right)^{\frac{1}{2}}}, \mathrm{~B} 2, \\
& \sin ^{2} \theta_{13}=\sin ^{2} \theta_{13}^{\circ}, \sin ^{2} \theta_{23}=\sin ^{2} \theta_{23}^{\circ}, \cos \delta-\text { unconstrained, B3, }
\end{aligned}
$$

where, as in the case of pattern $\mathbf{A}, \theta_{12}^{\circ}, \theta_{23}^{\circ}$ and $\theta_{13}^{\circ}$ are fixed once the symmetries are specified.

Finally, in the case of pattern $\mathbf{C}$, of which there are altogether nine sub-cases, corresponding to $U_{e}$ and $U_{v}^{\circ}$, each determined by $G_{e}$ and $G_{v}$ up to a unitary rotations in the $i-j$ and $k-l$ planes, respectively, $i-j=1-2,1-3,2-3, k-l=1-$ $2,1-3,2-3$, there is either a correlation between $\sin ^{2} \theta_{13}$ and $\sin ^{2} \theta_{12}$, or between $\sin ^{2} \theta_{13}$ and $\sin ^{2} \theta_{23}$, or else a sum rule for $\cos \delta$. We number them as in [14], i.e., cases C1-C9. Four of them lead to sum rules for $\cos \delta$, which have the form:

$\mathrm{C} 1,(i j, k l)=(12,13)$ :

$\cos \delta=\frac{\sin ^{2} \theta_{23}^{\circ}-\cos ^{2} \theta_{12} \sin ^{2} \theta_{23}-\cos ^{2} \theta_{23} \sin ^{2} \theta_{12} \sin ^{2} \theta_{13}}{\sin \theta_{13} \sin 2 \theta_{23} \sin \theta_{12} \cos \theta_{12}}$,

$\mathrm{C} 3,(i j, k l)=(12,23)$ :

$\cos \delta=\frac{\sin ^{2} \theta_{12} \sin ^{2} \theta_{23}-\sin ^{2} \theta_{13}^{\circ}+\cos ^{2} \theta_{12} \cos ^{2} \theta_{23} \sin ^{2} \theta_{13}}{\sin \theta_{13} \sin 2 \theta_{23} \sin \theta_{12} \cos \theta_{12}}$,

$\mathrm{C} 4,(i j, k l)=(13,23)$ :

$\cos \delta=\frac{\sin ^{2} \theta_{12}^{\circ}-\cos ^{2} \theta_{23} \sin ^{2} \theta_{12}-\cos ^{2} \theta_{12} \sin ^{2} \theta_{13} \sin ^{2} \theta_{23}}{\sin \theta_{13} \sin 2 \theta_{23} \sin \theta_{12} \cos \theta_{12}}$,

$\mathrm{C} 8,(i j, k l)=(13,13)$ :

$\cos \delta=\frac{\cos ^{2} \theta_{12} \cos ^{2} \theta_{23}-\cos ^{2} \theta_{23}^{\circ}+\sin ^{2} \theta_{12} \sin ^{2} \theta_{23} \sin ^{2} \theta_{13}}{\sin \theta_{13} \sin 2 \theta_{23} \sin \theta_{12} \cos \theta_{12}}$.

The neutrino mixing angles in these cases should be treated as free parameters. Other two cases, C5 and C 9 , yield correlations between $\sin ^{2} \theta_{12}$ and $\sin ^{2} \theta_{13}$ :

$\mathrm{C} 5,(i j, k l)=(23,13): \quad \sin ^{2} \theta_{12}=\frac{\sin ^{2} \theta_{12}^{\circ}}{1-\sin ^{2} \theta_{13}}$, $\cos \delta$ - unconstrained,

$\mathrm{C} 9,(i j, k l)=(23,23): \quad \sin ^{2} \theta_{12}=\frac{\sin ^{2} \theta_{12}^{\circ}-\sin ^{2} \theta_{13}}{1-\sin ^{2} \theta_{13}}$,

$\cos \delta$ - unconstrained.
In cases $\mathrm{C} 2$ and $\mathrm{C} 7$, instead, there are correlations between $\sin ^{2} \theta_{23}$ and $\sin ^{2} \theta_{13}$ :

$\mathrm{C} 2,(i j, k l)=(13,12): \quad \sin ^{2} \theta_{23}=\frac{\sin ^{2} \theta_{23}^{\circ}}{1-\sin ^{2} \theta_{13}}$,

$\cos \delta$ - unconstrained,

$\mathrm{C} 7,(i j, k l)=(12,12): \quad \sin ^{2} \theta_{23}=\frac{\sin ^{2} \theta_{23}^{\circ}-\sin ^{2} \theta_{13}}{1-\sin ^{2} \theta_{13}}$,

$\cos \delta$ - unconstrained.

Finally, in case $\mathrm{C} 6,(i j, k l)=(23,12), \cos \delta$ is unconstrained and $\sin ^{2} \theta_{13}$ is predicted to be equal to $\sin ^{2} \theta_{13}^{\circ}$. In cases $C 2$, $\mathrm{C} 5, \mathrm{C} 6, \mathrm{C} 7$ and $\mathrm{C} 9$, as is indicated above, $\cos \delta$ remains unconstrained.

Given the fact that the group $A_{4}$ has eight Abelian subgroups (three $Z_{2}$, four $Z_{3}$ and one Klein group $K_{4}$ isomorphic to $Z_{2} \times Z_{2}$ ), the group $S_{4}$ possesses 20 Abelian subgroups (nine $Z_{2}$, four $Z_{3}$, three $Z_{4}$ and four $Z_{2} \times Z_{2}$ groups, see, e.g., [11]), and $A_{5}$ has 36 Abelian subgroups (fifteen $Z_{2}$, ten $Z_{3}$, five $Z_{2} \times Z_{2}$ and six $Z_{5}$, see, e.g., [162]) the total number of the different residual symmetry patterns $\mathbf{A}$, $\mathbf{B}$ and $\mathbf{C}$ to be analysed is extremely large. For the group $A_{4}\left(T^{\prime}\right)$ alone there are altogether 64 cases (up to permutations of rows and columns of the predicted neutrino mixing matrix). It is quite remarkable that of these extremely large number of cases only a very limited number turned out to be phenomenologically viable, i.e., to be compatible with the existing data on the neutrino mixing angles $[14,42]$. In the case of the group $G_{f}=A_{4}\left(T^{\prime}\right)$, for example, only one case was found to be phenomenologically viable $[14,42]$, i.e., to be compatible with the experimentally determined values (including the $3 \sigma$ uncertainties) of the three neutrino mixing parameters $\sin ^{2} \theta_{12}, \sin ^{2} \theta_{13}$ and $\sin ^{2} \theta_{23}$. Namely, this is case B1 with $\left(G_{e}, G_{v}\right)=\left(Z_{3}, Z_{2}\right)$, which yields $\left(\sin ^{2} \theta_{12}^{\circ}, \sin ^{2} \theta_{23}^{\circ}\right)=(1 / 3,1 / 2)$ and corresponds to the TBM mixing matrix corrected from the right by the $U_{13}\left(\theta_{13}^{v}, \alpha\right)$ transformation in the 1-3 plane (see sub-section 3.3). The case B1 is common also to the two other groups $S_{4}$ 
and $A_{5}$. For $G_{f}=S_{4}$, there are 6 more viable cases. The $A_{5}$ flavour symmetry leads to 7 additional phenomenologically viable cases.

One arrives at this results for the number of phenomenologically viable cases in the following way. For the groups $S_{4}$ and $A_{5}$ there are respectively altogether 8 and 13 cases, which are acceptable a priori, i.e., which lead to $U_{\text {PMNS }}$ without zero entries. They are summarised in Table 3 (for $S_{4}$ ) and Table 4 (for $A_{5}$ ) of Ref. [42]. In Tables 3 and 4 in [42] the specific values of $\sin ^{2} \theta_{i j}^{\circ}$ in each case are also given. However, the case B1, as we have already noticed, is common to all the three flavour symmetry groups $A_{4}\left(T^{\prime}\right), S_{4}$ and $A_{5}$, while four cases, C1, C3, C4 and C8, are shared by $S_{4}$ and $A_{5}$. Thus, there are 16 cases in total, which lead to different predictions for $\sin ^{2} \theta_{12}$ or $\sin ^{2} \theta_{23}$ and/or $\cos \delta$. A statistical analysis of these predictions showed [42] that two cases, namely, $\mathrm{C} 4$ (for both $S_{4}$ and $A_{5}$ ) and B2A $\mathrm{A}_{5}$ II (i.e., the second of the two B2 cases with $G_{f}=A_{5}$, characterised by different fixed values of $\theta_{12}^{\circ}$ and $\theta_{13}^{\circ}$ ) are globally disfavored at more than $3 \sigma$ confidence level by the current data (including the uncertainties) on $\sin ^{2} \theta_{12}, \sin ^{2} \theta_{23}$ and $\sin ^{2} \theta_{13}$ [57]. As a consequence, only 14 cases altogether turned out to be phenomenologically viable at present. Five of them, B1, B1 $A_{5}$, $\mathrm{B} 2 S_{4}, \mathrm{~B} 2 A_{5}, \mathrm{C} 9 A_{5}{ }^{27}$, lead to sharp predictions for $\sin ^{2} \theta_{12}$, and four others, $\mathrm{A} 1 A_{5}, \mathrm{~A} 2 A_{5}, \mathrm{C} 2 S_{4}, \mathrm{C} 7 S_{4}$, to similarly sharp predictions for $\sin ^{2} \theta_{23}$. The six phenomenologically viable cases $\mathbf{A}$ and $\mathbf{B}$ lead also to predictions for $\cos \delta$, while five out of the eight viable cases $\mathbf{C}, \mathrm{C} 1, \mathrm{C} 3, \mathrm{C} 3 A_{5}$ (which differs from $\mathrm{C} 3$ that is common to $S_{4}$ and $A_{5}$ ), $\mathrm{C} 4 A_{5}$ (which differs from $\mathrm{C} 4$ ) and $\mathrm{C} 8$, also lead to predictions for $\cos \delta$.

Statistical analysis of these 14 cases was performed in [42] using the best fit values of the three neutrino mixing parameters $\sin ^{2} \theta_{i j}$ from [57] and taking into account the prospective $(1 \sigma)$ uncertainties in the determination of the mixing angles, planned to be achieved in currently running (Daya Bay $[159,160]$ ) and the next generation (JUNO [86], T2HK [101], DUNE [89]) of neutrino oscillation experiments: $3 \%$ on $\sin ^{2} \theta_{13}[159,160], 0.7 \%$ on $\sin ^{2} \theta_{12}$ [86] and $3 \%$ on $\sin ^{2} \theta_{23}[89,101]$. This analysis revealed that only six cases would be compatible with the indicated prospective data from the Daya Bay, JUNO, T2HK, DUNE neutrino oscillation experiments.

In Fig. 4, we present the likelihood functions for $\sin ^{2} \theta_{12}$ and $\sin ^{2} \theta_{23}$, obtained for NO and IO spectra in all the cases compatible at $3 \sigma$ with the current global data [57]. The corresponding likelihood profiles are very narrow because their widths are determined by the small experimental uncertainty

\footnotetext{
27 The notation $\mathrm{X} G_{f}$ means case $\mathrm{X}, \mathrm{X}=\mathrm{A} 1, \mathrm{~A} 2, \ldots, \mathrm{B} 1, \ldots, \mathrm{C} 1, \ldots, \mathrm{C} 9$, corresponding to the group $G_{f}, G_{f}=A_{4}, S_{4}, A_{5}$. The group is not indicated in cases B1, C1, C3 and C8 (see below) because case B1 is common to the $A_{4}, S_{4}$ and $A_{5}$ groups, while each of the cases C1, C3 and $\mathrm{C} 8$ is shared by the $S_{4}$ and $A_{5}$ groups.
}

on $\sin ^{2} \theta_{13}$. In the upper (lower) panel, the dashed line corresponds to the likelihood for $\sin ^{2} \theta_{12}\left(\sin ^{2} \theta_{23}\right)$ extracted from the global analysis. The dotted line represents the prospective precision on $\sin ^{2} \theta_{12}\left(\sin ^{2} \theta_{23}\right)$ corresponding to $1 \sigma$ uncertainty of $0.7 \%(3 \%)$, which is planned to be achieved by JUNO [86] (DUNE [89] and T2HK [101]). It is obtained under the assumption that the best fit value(s) of $\sin ^{2} \theta_{12}$ $\left(\sin ^{2} \theta_{23}\right)$ will not change in the future. If it is indeed the case, then, as is clear from Fig. 4, all five models leading to the predictions for $\sin ^{2} \theta_{12}$ will be ruled out by the JUNO measurement of this parameter.

The results of statistical analysis of the predictions for $\cos \delta$ are summarised in Fig. 5. The dashed line stands for the likelihood extracted from the global analysis [57]. At present, all (almost all) values of $\cos \delta$ are allowed at $3 \sigma$ for NO (IO) spectrum. We also show the dash-dotted and dotted lines which represent two benchmark cases. The first case, marked as "Future 1", corresponds to the current best fit NO (IO) value [57] $\delta^{\text {bf }}=234^{\circ}\left(278^{\circ}\right)$ and the prospective $1 \sigma$ uncertainty on $\delta$ of $10^{\circ}$. The second case, "Future 2 ", corresponds to the potential best fit value $\delta^{\text {bf }}=270^{\circ}$ (for both NO and IO cases) and the same $10^{\circ}$ error on $\delta$. The likelihoods in cases $C$ peak at values of $|\cos \delta| \sim 0.5-1$. Looking at the dotted line, we see that if in the future the best fit value of $\delta$ shifted to $270^{\circ}$ and the next generation of long-baseline experiments managed to achieve the $1 \sigma$ uncertainty on $\delta$ of $10^{\circ}$, all cases $\mathrm{C}$ viable at the moment would be disfavored at approximately $3 \sigma$ C.L. only by the measurement of $\delta$.

The results of the studies [14,42] summarised in the present subsection lead to the important conclusion that although the number of cases of non-Abelian discrete symmetry groups and their subgroups that can be used for description of lepton mixing is extremely large, only a very limited number survive when confronted with the existing data on the three neutrino mixing angles. This limited number of presently phenomenologically viable cases will be further considerably reduced by the precision measurements of the three neutrino mixing angles and the Dirac phase $\delta$ in the currently running (Daya Bay) and future planned (JUNO, T2HK, T2HKK, DUNE) neutrino oscillation experiments. As was shown in [42] and we have briefly discussed, if the best fit values of $\sin ^{2} \theta_{12}, \sin ^{2} \theta_{23}$ and $\sin ^{2} \theta_{13}$ as found in [57] would not change significantly in the future, only six cases would be compatible with the prospective data from the Daya Bay, JUNO, T2HK and DUNE neutrino oscillation experiments. This number would be further reduced by a precision measurement of the Dirac phase $\delta$.

\section{Flavour symmetry combined with generalised $\mathrm{CP}$ symmetry}

In all models discussed by us the Majorana phases $\alpha_{21}$ and $\alpha_{31}$ remain undetermined. The values of the Majorana CPV 

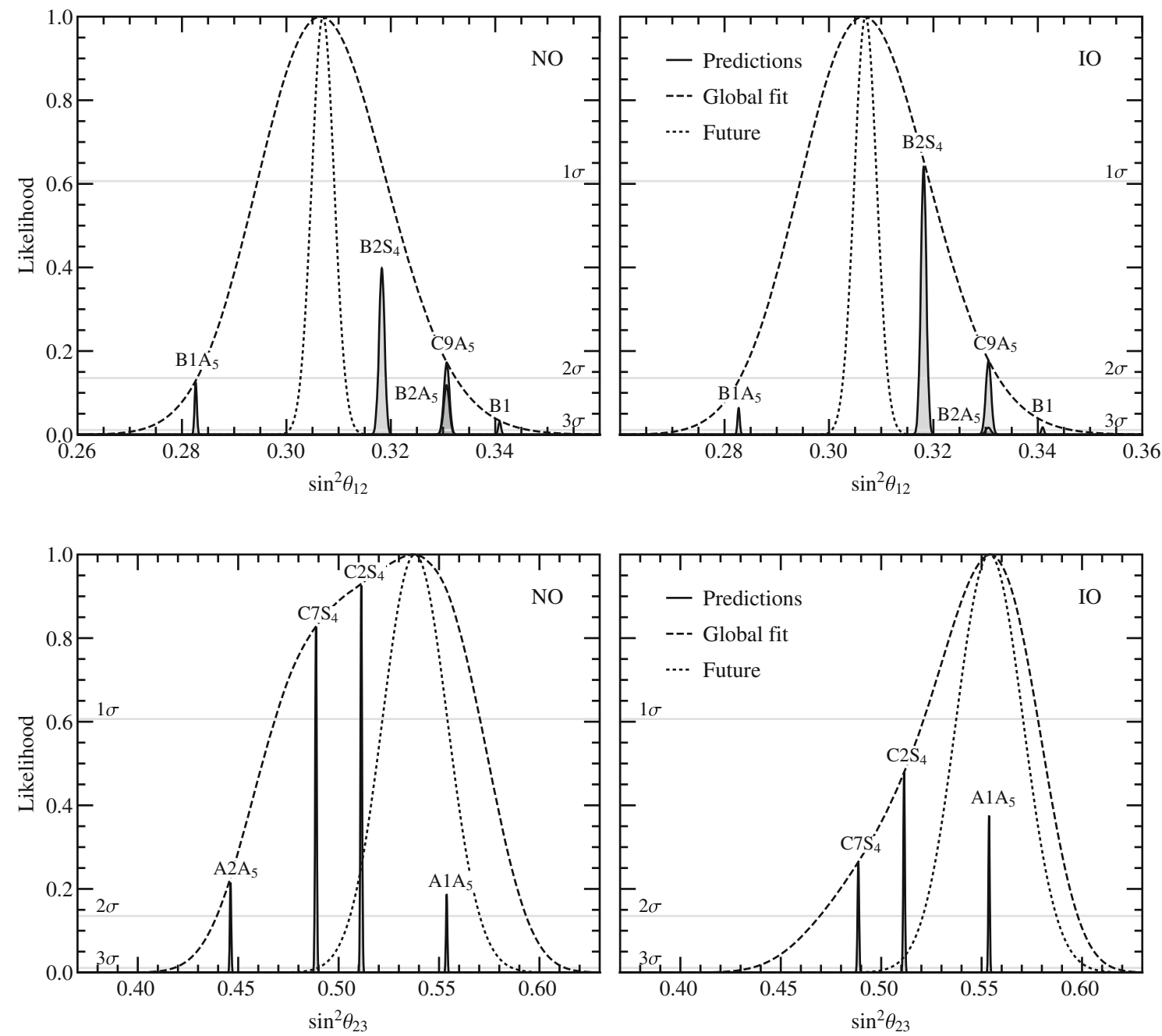

Fig. 4 Upper [lower] panel: predictions for $\sin ^{2} \theta_{12}$ [ $\sin ^{2} \theta_{23}$ ] obtained using the current global data on the neutrino mixing parameters. "Future" refers to the scenario with $\sin ^{2} \theta_{12}^{\text {bf }}=0.307\left[\sin ^{2} \theta_{23}^{\text {bf }}=\right.$

phases are instead constrained to lie in certain narrow intervals, or are predicted, in theories which in addition to a flavour symmetry possess at a certain high-energy scale a "generalised CP" (GCP) symmetry [21]. The GCP symmetry, as the term suggests, is a generalisation of the traditional (canonical) CP symmetry. The GCP symmetry should be implemented in a theory based on a discrete flavour symmetry in a way that is consistent with the flavour symmetry $[22,23]$. At low energies the GCP symmetry is broken, in general, to residual $\mathrm{CP}$ symmetries of the charged lepton and neutrino sectors.

The GCP transformations are applied on the $\mathrm{LH}$ and RH components of the charged lepton fields $\tilde{l}_{L}(x)$ and $\tilde{l}_{R}(x)$ and on the LH neutrino fields $v_{\tilde{l} L}(x)$ - the fields in terms of which the general charged lepton and neutrino Majorana mass terms are formed, Eqs. (9) and (11). The transformations of interest are defines as follows:

$0.538(0.554)$ for $\mathrm{NO}(\mathrm{IO})]$ (current best fit values) and the relative $1 \sigma$ uncertainty of $0.7 \%$ [3\%] expected from JUNO [DUNE and T2HK]. See text for further details. (From Ref. [42].)

$\tilde{l}_{L}(x) \stackrel{C P}{\longrightarrow} i\left(X_{L}\right)_{\tilde{l} l^{\prime}} \gamma_{0} C{\overline{\tilde{l}_{L}^{\prime}\left(x^{\prime}\right)}}^{T}$,

$\tilde{l}_{R}(x) \stackrel{C P}{\longrightarrow} i\left(X_{R}\right)_{\tilde{l l} l^{\prime}} \gamma_{0} C{\overline{\tilde{l}_{R}^{\prime}\left(x^{\prime}\right)}}^{T}$,

$v_{\tilde{l} L}(x) \stackrel{C P}{\longrightarrow} i\left(X_{L}\right)_{\tilde{l l^{\prime}}} \gamma_{0} C{\overline{v_{\tilde{l}^{\prime} L}\left(x^{\prime}\right)}}^{T}$,

where $\tilde{l}=\tilde{e}, \tilde{\mu}, \tilde{\tau}, X_{L}$ and $X_{R}$ are $3 \times 3$ unitary matrices and $x^{\prime}=(t,-\mathbf{x})$. The transformations of $\tilde{l}_{L}(x)$ and $v_{\tilde{l}_{L}}(x)$ should involve the same matrix $X_{L}$ in order to ensure the CP invariance of the $\mathrm{CC}$ weak interaction Lagrangian, expressed in terms of the SM $S U(2)_{L}$ lepton doublet fields $\tilde{l}_{L}(x)$ and $v_{\tilde{l} L}(x)$ :

$\mathcal{L}_{\mathrm{CC}}=-\frac{g}{\sqrt{2}} \sum_{\tilde{l}=\tilde{e}, \tilde{\mu}, \tilde{\tau}} \overline{\tilde{l}_{L}(x)} \gamma_{\alpha} v_{\tilde{l} L}(x) W^{\alpha \dagger}(x)+$ h.c..

The GCP symmetry will hold then in the lepton sector if it is a symmetry of the charged lepton and neutrino mass terms, 

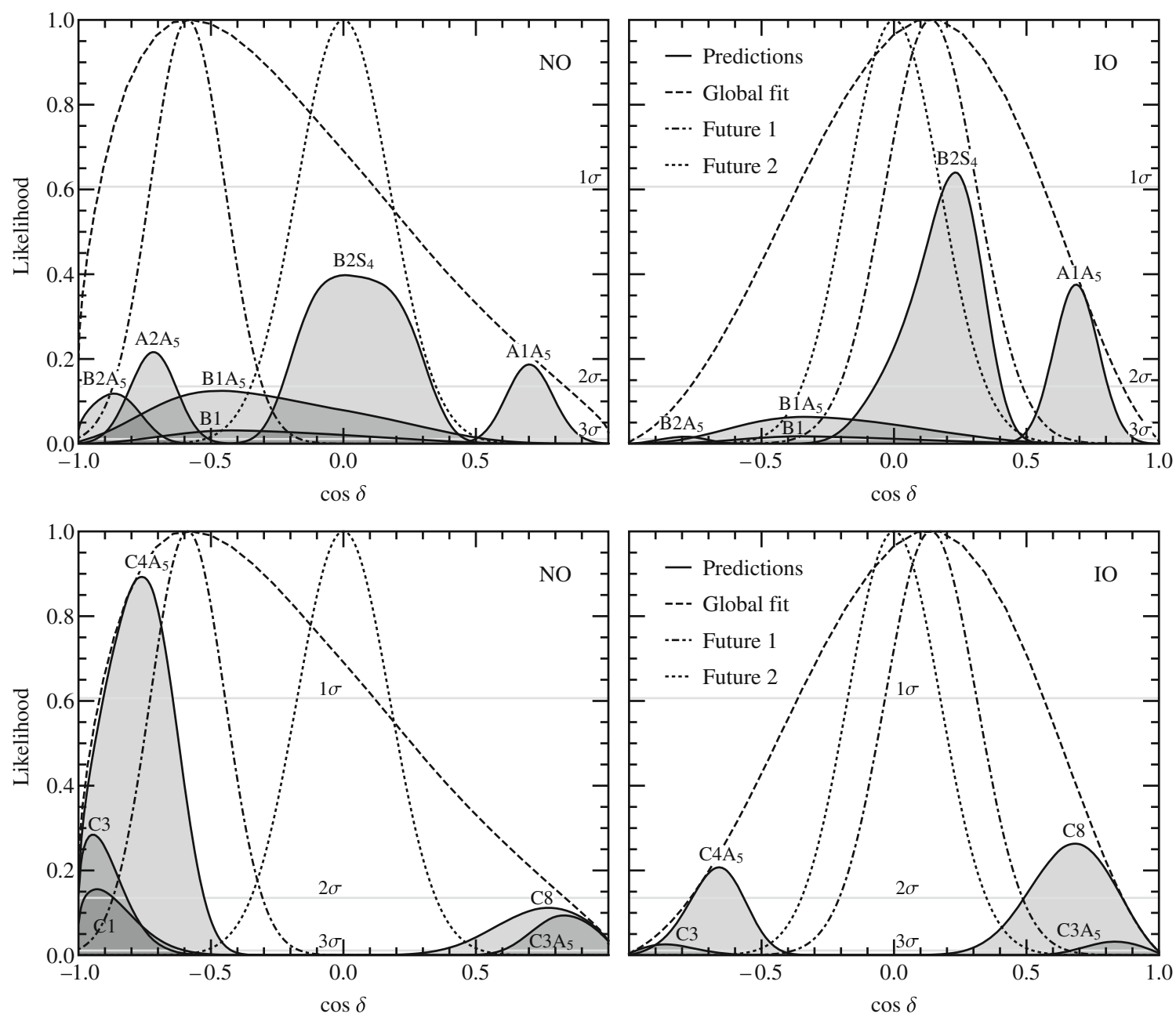

Fig. 5 Predictions for $\cos \delta$ obtained using the current global data on the neutrino mixing parameters. "Future 1 " refers to the scenario with $\delta^{\text {bf }}=234^{\circ}\left(278^{\circ}\right)$ for NO (IO) spectrum (current best fit values) and

Eqs. (9) and (11), i.e., if the charged lepton and neutrino Majorana mass matrices satisfy the following constraints:

$$
\begin{aligned}
X_{L}^{\dagger} M_{e} X_{R} & =M_{e}^{*}, \text { or } \\
X_{L}^{\dagger} M_{e} M_{e}^{\dagger} X_{L} & =\left(M_{e} M_{e}^{\dagger}\right)^{*}, \\
X_{L}^{T} M_{v} X_{L} & =M_{v}^{*} .
\end{aligned}
$$

In the presence of flavour symmetry the form of the GCP transformations is significantly constrained. Indeed, consider a GCP transformation on a generic field $\varphi(x)$ which is assigned to an $\mathrm{r}$-dimensional irreducible unitary representation $\rho_{r}(g)$ of $G_{f}$ :

$\varphi(x) \stackrel{C P}{\longrightarrow} X_{r} \varphi^{*}\left(x^{\prime}\right)$,

where $X_{r}$ is a unitary matrix. The action of the CP transformation on the spinor indices in the case of $\varphi$ being a spinor the $1 \sigma$ uncertainty on $\delta$ of $10^{\circ}$. "Future 2 " corresponds to $\delta^{\text {bf }}=270^{\circ}$ and the $1 \sigma$ uncertainty on $\delta$ of $10^{\circ}$. See text for further details. (From Ref. [42].)

[shown explicitly in Eqs. (91)-(93)] has been omitted here for simplicity. If both the flavour symmetry and the GCP symmetry hold, the theory under study should be invariant also under the following sequence of transformations: a GCP transformation, followed by a flavour symmetry transformation, which in turn is followed by an (inverse) GCP transformation, i.e., under

$$
\begin{aligned}
\varphi(x) & \stackrel{C P}{\longrightarrow} X_{r} \varphi^{*}\left(x^{\prime}\right) \stackrel{G_{f}}{\longrightarrow} X_{r} \rho_{r}\left(g_{f}\right)^{*} \varphi^{*}\left(x^{\prime}\right) \\
& \stackrel{C P^{-1}}{\longrightarrow} X_{r} \rho_{r}\left(g_{f}\right)^{*} X_{r}^{-1} \varphi(x) .
\end{aligned}
$$

In order for the theory to be invariant under this sequence of transformations the resulting transformation should be a flavour symmetry transformation of $\varphi(x)$ corresponding to an element $g_{f}^{\prime}$ of $G_{f}$, which can differ from $g_{f}$ : 
$X_{r} \rho_{r}\left(g_{f}\right)^{*} X_{r}^{-1}=\rho_{r}\left(g_{f}^{\prime}\right), \quad g_{f}, g_{f}^{\prime} \in G_{f}$.

This equation represents a consistency condition which has to be satisfied in order for the implementation of the GCP symmetry in the theory to be compatible with the presence of a flavour symmetry [22,23]. For a discrete flavour symmetry group $G_{f}$, the consistency condition (100) will hold if it is satisfied by the group's generators.

Let us denote by $H_{\mathrm{CP}}=\left\{X_{L}\right\}$ the full set of GCP transformations $X_{L}$ acting on $v_{\tilde{l} L}(x)$ and $\tilde{l}_{L}(x)$, which are compatible with a given flavour symmetry group $G_{f}$, i.e., which satisfy the consistency condition (100) in which $X_{r}$ is replaced by $X_{L}$ and $\rho_{r}\left(g_{f}\right)$ is the irreducible unitary representation of $G_{f}$ to which $v_{\tilde{l} L}(x)$ and $\tilde{l}_{L}(x)$ are assigned. We denote further by $H_{\mathrm{CP}}^{v}=\left\{X_{v}\right\}$ and $H_{\mathrm{CP}}^{\ell}=\left\{X_{\ell}\right\}$ the sets of GCP transformations $X_{v}$ and $X_{\ell}$ which are compatible respectively with the residual flavour symmetries $G_{\nu}$ and $G_{\ell}$ of the neutrino and charged lepton sectors, i.e., of the neutrino Majorana and charged lepton mass terms. $X_{v}$ and $X_{\ell}$ satisfy consistency conditions in which $\rho_{r}\left(g_{f}\right)\left(\rho_{r}\left(g_{f}^{\prime}\right)\right)$ is replaced respectively by $\rho_{r}\left(g_{v}\right)\left(\rho_{r}\left(g_{v}^{\prime}\right)\right)$ and $\rho_{r}\left(g_{\ell}\right)\left(\rho_{r}\left(g_{\ell}^{\prime}\right)\right)$, where $g_{v}, g_{v}^{\prime} \in G_{v}$ and $g_{\ell}, g_{\ell}^{\prime} \in G_{\ell}$.

The sets $H_{\mathrm{CP}}, H_{\mathrm{CP}}^{v}$ and $H_{\mathrm{CP}}^{\ell}$ are not groups by themselves. They are sets of GCP transformations, which always involve conjugation of the fields they act upon; $H_{\mathrm{CP}}^{v}$ and $H_{\mathrm{CP}}^{\ell}$ are subsets of $H_{\mathrm{CP}}$. These sets become groups only if they are extended by (at least) an identity element which does not conjugate fields (see, for instance, Appendix B of Ref. [22]). When one writes a semi-direct product of $G_{f}$ and $H_{\mathrm{CP}}$, $G_{f} \rtimes H_{\mathrm{CP}}$, and the semi-direct ${ }^{28}$ products of $G_{v}$ and $H_{\mathrm{CP}}^{v}$, $G_{v} \rtimes H_{\mathrm{CP}}^{\nu}$, and of $G_{\ell}$ and $H_{\mathrm{CP}}^{\ell}, G_{\ell} \rtimes H_{\mathrm{CP}}^{\ell}$, it is always implicitly assumed that $H_{\mathrm{CP}}, H_{\mathrm{CP}}^{v}$ and $H_{\mathrm{CP}}^{\ell}$ are appropriate groups, which are obtained from a single generating GCP transformation, as explained in Appendix B of Ref. [22]. In this case $H_{\mathrm{CP}}^{v}$ and $H_{\mathrm{CP}}^{\ell}$ are subgroups of $H_{\mathrm{CP}}$.

\subsection{Implications for the majorana phases}

As we have discussed in Sect. 3.1, the unitary matrix $U_{v}$ which diagonalises the neutrino Majorana mass matrix $M_{v}$ and enters into the expression for the PMNS matrix, Eq. (8), is related to the unitary matrix $U_{v}^{\circ}$ diagonalising $M_{v}^{\dagger} M_{v}$ and $\rho_{r}\left(g_{v}\right)$ in the following way: $U_{v} \equiv U_{v}^{\circ} P^{\circ}$, where $P^{\circ}=\operatorname{diag}\left(1, e^{i \frac{\xi_{21}}{2}}, e^{i \frac{\xi_{31}}{2}}\right.$ ) (see Eq. (20)). The phases $\xi_{21}$ and $\xi_{31}$ contribute respectively to the Majorana phases $\alpha_{21}$ and $\alpha_{31}$ of the PMNS matrix, Eq. (2). These phases remain undetermined by the flavour symmetries under discussion. We will consider next the implications of a residual GCP symmetry $H_{\mathrm{CP}}^{v} \subset H_{\mathrm{CP}}$, which is preserved in the neutrino

\footnotetext{
${ }^{28}$ In the case of $G_{v}$ or $G_{e}$ being a $Z_{2}$ symmetry, the corresponding product becomes direct.
}

sector, for the determination of $\xi_{21}$ and $\xi_{31}$, and thus of the Majorana phases $\alpha_{21}$ and $\alpha_{31}$.

In the case of a residual GCP symmetry $H_{\mathrm{CP}}^{v}$, the neutrino Majorana mass matrix satisfies the condition given in Eq. (97), $X_{L} \in H_{\mathrm{CP}}^{v}$ being the GCP transformation defined in Eq. (93). Using Eq. (12) we find:

$$
\begin{gathered}
\left(X_{L}^{\mathrm{d}}\right)^{T} M_{v}^{\mathrm{d}} X_{L}^{\mathrm{d}}=M_{v}^{\mathrm{d}}, \quad \text { with } M_{v}^{\mathrm{d}}=\operatorname{diag}\left(m_{1}, m_{2}, m_{3}\right), \\
X_{L}^{\mathrm{d}}=U_{v}^{\dagger} X_{L} U_{v}^{*} .
\end{gathered}
$$

For $m_{1} \neq m_{2} \neq m_{3}$ and $^{29} \min \left(m_{j}\right) \neq 0, j=1,2,3$, as it is not difficult to show (see, e.g., $[22,33,165])$, the unitary matrix $X_{L}^{\mathrm{d}}$ can have only the following form:

$X_{L}^{\mathrm{d}}=\operatorname{diag}( \pm 1, \pm 1, \pm 1)$,

where the signs of the three non-zero entries in $X_{L}^{\mathrm{d}}$ are not correlated. Further, using that $U_{v}=U_{v}^{\circ} P^{\circ}$, we obtain from Eq. (101) [165]:

$$
\begin{aligned}
& \left(U_{v}^{\circ}\right)^{\dagger} X_{L}\left(U_{v}^{\circ}\right)^{*}=P^{\circ} X_{L}^{\mathrm{d}} P^{\circ} \\
& =\operatorname{diag}\left( \pm 1, \pm e^{i \xi_{21}}, \pm e^{i \xi_{31}}\right) .
\end{aligned}
$$

Thus, we come to the conclusion that the phases $\xi_{21}$ and $\xi_{31}$ will be determined provided i) the matrix $U_{v}^{\circ}$, which diagonalises $M_{v}^{\dagger} M_{v}$ and $\rho_{r}\left(g_{v}\right)$ (see Eq. (19) and the related discussion) is fixed by the residual flavour symmetry $G_{\nu}$, and (ii) the GCP transformations $X_{L} \in H_{\mathrm{CP}}^{v}$, which are consistent with $G_{v}$, are identified.

\subsection{Concrete examples of symmetries}

Now we turn to concrete examples [33]. For $G_{f}=A_{4}$ we choose to work in the Altarelli-Feruglio basis [143], in which the $S$ and $T$ generators have the form given in Eq. (24). Preserving the $S$ generator, i.e., choosing $G_{v}=Z_{2}^{S}=\{1, S\}$, leads to $U_{v}^{\circ}=V_{\mathrm{TBM}}$, provided there is an additional accidental $\mu-\tau$ symmetry [9]. The twelve GCP transformations consistent with the $A_{4}$ flavour symmetry for the triplet representation in the chosen basis have been found in [26], solving the consistency condition

$X_{L} \rho^{*}(g) X_{L}^{-1}=\rho\left(g^{\prime}\right), \quad g, g^{\prime} \in A_{4}$.

These transformations can be summarised in a compact way as follows:

$X_{L}=\rho(g), \quad g \in A_{4}$,

\footnotetext{
29 It follows from the neutrino oscillation data that $m_{1} \neq m_{2} \neq m_{3}$, and that at least two of the three neutrino masses, $m_{2,3}\left(m_{1,2}\right)$ in the case of the NO (IO) spectrum, are non-zero. However, even if $m_{1}=0\left(m_{3}=0\right)$ at tree level and the zero value is not protected by a symmetry, $m_{1}\left(m_{3}\right)$ will get a non-zero contribution at least at two loop level $[163,164]$ and in the framework of a self-consistent (renormalisable) theory of neutrino mass generation this higher order contribution will be finite.
} 
i.e., the GCP transformations consistent with the $A_{4}$ flavour symmetry are of the same form as the flavour symmetry group transformations [26]. They are given in Table 1 in [26] together with the elements $\hat{S}$ and $\hat{T}$ to which the generators $S$ and $T$ of $A_{4}$ are mapped by the consistency condition in Eq. (104). Further, since in our case the residual flavour symmetry $G_{v}=Z_{2}^{S} \times Z_{2}$, where the $Z_{2}^{S}$ factor corresponds to the preserved $S$ generator, only those $X$ are acceptable, for which $\hat{S}=S$. From Table 1 in [26] it follows that there are four such GCP transformations, namely, $\rho(E), \rho(S), \rho\left(T^{2} S T\right)$ and $\rho\left(T S T^{2}\right)$, where $E$ is the identity element of the group. The last two transformations are not symmetric in the chosen basis, and, as shown in [26], lead to partially degenerate neutrino mass spectrum with two equal masses (see also [22]), which is ruled out by the existing neutrino oscillation data. Thus, we are left with two allowed generalised CP transformations, $\rho(E)$ and $\rho(S)$, for which we have:

$$
\begin{aligned}
& V_{\mathrm{TBM}}^{\dagger} \rho(E) V_{\mathrm{TBM}}^{*}=\rho(E)=\operatorname{diag}(1,1,1), \\
& V_{\mathrm{TBM}}^{\dagger} \rho(S) V_{\mathrm{TBM}}^{*}=\operatorname{diag}(-1,1,-1) .
\end{aligned}
$$

Finally, according to Eq. (103), this implies that the phases $\xi_{21}$ and $\xi_{31}$ can be either 0 or $\pi$. The same conclusion holds for a $T^{\prime}$ flavour symmetry, because restricting ourselves to the triplet representation for the $\mathrm{LH}$ charged lepton and neutrino fields, there is no way to distinguish $T^{\prime}$ from $A_{4}$ [156].

In the case of $G_{f}=S_{4}$ considered in [33], the authors chose to work with the two generators $S$ and $T$ of $S_{4}$ in the basis given in [136]. In this case the generators $S$ and $T$ satisfy the following presentation rules:

$S^{2}=T^{4}=(S T)^{3}=(T S)^{3}=\mathbf{1}$.

Although the presentation rules for $S$ and $T$ given above differ from the presentation rules when the third generator $U$ for $S_{4}$ is employed, Eq. (23), we will keep the notation $S$ and $T$ for the two generators satisfying the presentation rules (108) in the discussion which follows. In the basis chosen in [136] and used in [33], $S$ and $T$ have the following form in the two triplet representations of interest:

$$
\begin{aligned}
& S= \pm\left(\begin{array}{ccc}
0 & -\frac{1}{\sqrt{2}} & -\frac{1}{\sqrt{2}} \\
-\frac{1}{\sqrt{2}} & \frac{1}{2} & -\frac{1}{2} \\
-\frac{1}{\sqrt{2}} & -\frac{1}{2} & \frac{1}{2}
\end{array}\right), \\
& T= \pm\left(\begin{array}{ccc}
-1 & 0 & 0 \\
0 & -i & 0 \\
0 & 0 & i
\end{array}\right),
\end{aligned}
$$

The residual symmetry $G_{v}=Z_{2}^{S} \times Z_{2}$, where the $Z_{2}^{S}$ factor corresponds to preserved $S$ generator in the chosen basis
Table 5 The ten symmetric generalised CP transformations $X=\rho(g)$ consistent with the $S_{4}$ flavour symmetry for the triplet representation $\rho$ in the chosen basis [136], determined by the consistency condition in Eq. (100). The mapping $(T, S) \rightarrow(\hat{T}, \hat{S})$ is realised via the consistency condition applied to the group generators $T$ and $S$, i.e., $X \rho^{*}(T) X^{-1}=$ $\rho(\hat{T})$ and $X \rho^{*}(S) X^{-1}=\rho(\hat{S}) . E$ denotes the identity element of $S_{4}$. (From [33].)

\begin{tabular}{lll}
\hline$g, X=\rho(g)$ & $T \rightarrow \hat{T}$ & $S \rightarrow \hat{S}$ \\
\hline$\left(S T^{2}\right)^{2}$ & $T$ & $S$ \\
$T^{3}$ & $T^{3}$ & $T^{3} S T$ \\
$E$ & $T^{3}$ & $S$ \\
$T$ & $T^{3}$ & $T S T^{3}$ \\
$T^{2} S T^{2}$ & $S T S$ & $S$ \\
$S T^{2} S$ & $T$ & $T^{2} S T^{2}$ \\
$S$ & $T S T$ & $S$ \\
$T^{2}$ & $T^{3}$ & $T^{2} S T^{2}$ \\
$S T S$ & $S T^{2}$ & $S T^{2} S T$ \\
$T S T$ & $T^{2} S$ & $T S T^{2} S$ \\
\hline
\end{tabular}

and the second one arises accidentally (corresponding to a $\mu-\tau$ symmetry), leads to the bimaximal mixing, $U_{v}^{\circ}=V_{\mathrm{BM}}$ [136]. As in the previous example, the GCP transformations consistent with the $S_{4}$ flavour symmetry are of the same form as the flavour symmetry group transformations [23]. Solving the consistency condition in Eq. (104), in [33] ten symmetric GCP transformations consistent with the $S_{4}$ flavour symmetry for the triplet representation in the chosen basis were found. They are summarised in Table 5 together with the elements $\hat{T}$ and $\hat{S}$ to which the consistency condition maps the group generators $T$ and $S$.

From Table 5 we see that there are four symmetric GCP transformations consistent with the preserved $S$ generator, namely, $\rho(E), \rho(S), \rho\left(T^{2} S T^{2}\right)$ and $\rho\left(S T^{2} S T^{2}\right)$. Substituting them and $U_{v}^{\circ}=V_{\mathrm{BM}}$ in Eq. (103), one finds [33]:

$$
\begin{aligned}
V_{\mathrm{BM}}^{\dagger} \rho(E) V_{\mathrm{BM}}^{*} & =\rho(E)=\operatorname{diag}(1,1,1), \\
V_{\mathrm{BM}}^{\dagger} \rho(S) V_{\mathrm{BM}}^{*} & =\operatorname{diag}(1,-1,1), \\
V_{\mathrm{BM}}^{\dagger} \rho\left(T^{2} S T^{2}\right) V_{\mathrm{BM}}^{*} & =\operatorname{diag}(-1,1,1), \\
V_{\mathrm{BM}}^{\dagger} \rho\left(S T^{2} S T^{2}\right) V_{\mathrm{BM}}^{*} & =\operatorname{diag}(-1,-1,1) .
\end{aligned}
$$

Therefore also in this case the phases $\xi_{21}$ and $\xi_{31}$ are fixed by the residual GCP symmetry to be either 0 or $\pi$. As was shown in [33], these results for the phases $\xi_{21}$ and $\xi_{31}$ hold also for $G_{f}=A_{5}$ and $G_{v}=Z_{2} \times Z_{2}$, generated by $\tilde{S}$ and $\tilde{T}^{3} \tilde{S} \tilde{T}^{2} \tilde{S} \tilde{T}^{3}$ and leading to the GRA mixing, $U_{v}^{\circ}=U_{\mathrm{GRA}}$ (see Sect. 3.6), when the flavour symmetry is combined with the GCP symmetry.

If the matrix $U_{e}$ originating from the charged lepton sector is non-trivial, as like in the cases $\mathbf{A}$ and $\mathbf{B}$ defined by equations (66), (67) and (68), the Majorana phases $\alpha_{21}$ and $\alpha_{31}$ of 
the PMNS matrix receive also contributions from the phases associated with $U_{e}$ in the PMNS matrix [12]. In the specific examples of the forms $\mathbf{A}$ and $\mathbf{B}$ of $U_{e}$, the phases $\omega$ and/or $\psi$ of the matrix $\Psi$ in Eqs. (66) and (68), as we have already remarked, serve as a source for the Dirac CPV phase $\delta$ of the PMNS matrix and contribute to the Majorana phases $\alpha_{21}$ and $\alpha_{31}$ [12]. In these cases the Majorana phases $\alpha_{21}$ and $\alpha_{31}$ are determined by the sums respectively of the phases $\xi_{21}$ and $\xi_{31}$ and of the indicated additional contributions due to the phases in the matrix $\Psi$. As a consequence, $\alpha_{21}$ and $\alpha_{31}$ have non-trivial values which differ from 0 or $\pi$ even when $\xi_{21}$ and $\xi_{31}$ are fixed by the employed residual GCP symmetry to be either 0 or $\pi[12,33]$.

As we have indicated earlier, the Majorana phases play important role, e.g, in $|\Delta L|=2$ processes like neutrinoless double beta $\left((\beta \beta)_{0 v^{-}}\right)$decay $(A, Z) \rightarrow(A, Z+2)+e^{-}+e^{-}$, $L$ being the total lepton charge, in which the Majorana nature of massive neutrinos $v_{i}$ manifests itself (see, e.g, Ref. [1]). Determining the values of the Majorana phases allows to make predictions for the basic $(\beta \beta)_{0 v}$ - decay parameter the effective neutrino Majorana mass (see, e.g, Refs. [12,33, 35,53]).

\subsection{Examples of models}

In the scenarios involving a GCP symmetry, which were most widely explored so far (see, e.g., [22,24,26,28-30]), a non-Abelian flavour symmetry $G_{f}$ consistently combined with a GCP symmetry $H_{\mathrm{CP}}$ is broken to residual Abelian symmetries $G_{e}=Z_{n}, n>2$, or $Z_{m} \times Z_{k}, m, k \geq 2$, and $G_{v}=Z_{2} \times H_{\mathrm{CP}}^{v}$ of the charged lepton and neutrino mass terms, respectively. ${ }^{30}$ The factor $H_{\mathrm{CP}}^{v}$ in $G_{v}$ stands for a remnant GCP symmetry of the neutrino mass term. In such a set-up, $G_{e}$ fixes completely the form of the unitary matrix $U_{e}$ which diagonalises the product $M_{e} M_{e}^{\dagger}$ and enters into the expression of the PMNS matrix. At the same time, $G_{v}$ fixes the unitary matrix $U_{v}$, diagonalising the neutrino Majorana mass matrix $M_{v}$ up to a single free real parameter - a rotation angle $\theta^{v}$. Given the fact that the PMNS neutrino mixing matrix $U_{\text {PMNS }}$ is given by the product $U_{\mathrm{PMNS}}=U_{e}^{\dagger} U_{v}$, all three neutrino mixing angles are expressed in terms of this rotation angle. In this class of models one obtains specific correlations between the values of the three neutrino mixing angles, while the leptonic CPV phases are typically predicted to be exactly 0 or $\pi$, or else $\pi / 2$ or $3 \pi / 2$. For example, in the set-up considered in [22] and based on $G_{f} \rtimes H_{\mathrm{CP}}=S_{4} \rtimes H_{\mathrm{CP}}$ broken to $G_{e}=Z_{3}^{T}$

\footnotetext{
$\overline{30}$ We note that in Refs. $[26,28]$ the residual symmetry $G_{e}$ of the charged lepton mass term is augmented with a remnant $\mathrm{CP}$ symmetry $H_{\mathrm{CP}}^{\ell}$ as well.
}

and $G_{v}=Z_{2}^{S} \times H_{\mathrm{CP}}^{\nu}$ with $H_{\mathrm{CP}}^{v}=\{U, S U\},{ }^{31}$ the authors find:

$$
\begin{aligned}
\sin ^{2} \theta_{13} & =\frac{2}{3} \sin ^{2} \theta^{v}, \quad \sin ^{2} \theta_{12}=\frac{1}{2+\cos 2 \theta^{v}} \\
& =\frac{1}{3\left(1-\sin ^{2} \theta_{13}\right)}, \quad \sin ^{2} \theta_{23}=\frac{1}{2}, \\
|\sin \delta| & =1, \quad \sin \alpha_{21}=\sin \alpha_{31}=0 .
\end{aligned}
$$

It follows, in particular, from the results on the neutrino oscillation parameters - best fit values, $2 \sigma$ and $3 \sigma$ allowed ranges - obtained in the global fit of neutrino oscillation data [54] and summarised in Table 1, as well as in the more recent analyses [57,58], that the prediction quoted in Eq. (114) for $\sin ^{2} \theta_{12}$ lies outside of its currently allowed $2 \sigma$ range. ${ }^{32}$ In what concerns the prediction $\sin ^{2} \theta_{23}=1 / 2$, according to $[57,58]$, it lies within the $1 \sigma(2 \sigma)$ allowed range of $\sin ^{2} \theta_{23}$ for NO (IO) spectrum.

Other examples of one (real angle) parameter models based on the flavour symmetry groups $A_{4}, S_{4}$ and $A_{5}$ combined with GCP symmetry can be found, e.g., in Refs. $[24,26,28-31,166]$. Most of them share some of the properties of the model discussed above: the correlation between $\sin ^{2} \theta_{12}$ and $\sin ^{2} \theta_{13}$, the predictions that $|\sin \delta|=1$, $\sin \alpha_{21}=\sin \alpha_{31}=0$. Some of the models predict $\sin \delta=0$, which is disfavored by the results of the global neutrino data analyses (see Table 1). In certain set-ups the Majorana phases $\alpha_{21}$ and $\alpha_{31}$ take non-trivial values while $|\sin \delta|=1$.

In a class of models based on the groups $G_{f}=\Delta\left(3 n^{2}\right)$ and $G_{f}=\Delta\left(6 n^{2}\right)$ of flavour symmetry combined with GCP symmetry the neutrino mixing angles and the CPV phases are functions of one real angle and one or two discrete phase parameters, which depend on the parameter $n$ which characterises the size of the group $G_{f}$ (see, e.g., [27,68,167-169]). Due to the presence of the additional discrete valued phases, the CPV phase $\delta$ can have non-trivial and non-maximal values, i.e., one can have $|\sin \delta| \neq 1,0$. In this class of models, as a rule, there exist correlations between the values of $\sin ^{2} \theta_{12}, \sin ^{2} \theta_{23}$ and of $\sin ^{2} \theta_{13}$ in the form of, e.g., the following relations [168,169]: $3 \cos ^{2} \theta_{13} \sin ^{2} \theta_{12}=1$ and $\sin ^{2} \theta_{23}=0.5 \pm 0.5 \tan \theta_{13} \sqrt{2-\tan ^{2} \theta_{13}}$. The first correlation $3 \cos ^{2} \theta_{13} \sin ^{2} \theta_{12}=1$ is typical for the models under discussion, while the second one or similar occur in most of them. For the best fit value of $\sin ^{2} \theta_{13}$ given in Table 1 , the quoted relations lead to the predictions: $\sin ^{2} \theta_{12}=0.340$ and $\sin ^{2} \theta_{23}=0.396$ or 0.604 . As we have already noticed, the

\footnotetext{
${ }^{31}$ We recall that $S, T$ and $U$ are the generators of $S_{4}$ in the basis for its 3-dimensional representation specified in Eq. (24).

32 We have used the best fit value of $\sin ^{2} \theta_{13}$ to obtain the prediction of $\sin ^{2} \theta_{12}=0.341$ leading to the quoted conclusion. Using the $2 \sigma$ allowed range for $\sin ^{2} \theta_{13}$ leads to a minimal value of $\sin ^{2} \theta_{12}=0.340$, which is still larger than the maximal allowed value of $\sin ^{2} \theta_{12}$ at $2 \sigma$ C.L., but inside its $3 \sigma$ allowed range.
} 
value $\sin ^{2} \theta_{12}=0.340$ is outside the $2 \sigma$, but within the $3 \sigma$ allowed ranges of $\sin ^{2} \theta_{12}$ found in [54,57,58]. As it follows from the results reported, e.g., in [58], both predicted values of $\sin ^{2} \theta_{23}$ lie outside the $3 \sigma$ allowed range of $\sin ^{2} \theta_{23}$.

Theoretical models based on the approach to neutrino mixing that combines discrete symmetries and GCP invariance, in which the neutrino mixing angles and the leptonic CPV phases are functions of two or three parameters have also been considered in the literature (see, e.g., [25,32-35]). In these models the residual symmetry $G_{e}$ of the charged lepton mass term is typically assumed to be a $Z_{2}$ symmetry or to be fully broken. In spite of the larger number of parameters in terms of which the neutrino mixing angles and the leptonic $\mathrm{CPV}$ phases are expressed, the values of the CPV phases are still predicted to be correlated with the values of the three neutrino mixing angles. A set-up with $G_{e}=Z_{2} \times H_{\mathrm{CP}}^{e}$ and $G_{v}=Z_{2} \times H_{\mathrm{CP}}^{v}$ has been considered in [34]. The resulting PMNS matrix in such a scheme depends on two free real parameters - two angles $\theta^{v}$ and $\theta^{e}$. The authors have obtained several phenomenologically viable neutrino mixing patterns from $G_{f}=S_{4}$ combined with $H_{\mathrm{CP}}$, broken to all possible residual symmetries of the type indicated above. Models allowing for three free parameters (two real angles and one phase) have been investigated in $[25,32,33,35]$. In, e.g., [32], the author has considered $G_{f}=A_{5}$ combined with $H_{\mathrm{CP}}$, which are broken to $G_{e}=Z_{2}$ and $G_{v}=Z_{2} \times H_{\mathrm{CP}}^{v}$. In this case, the matrix $U_{e}$ depends on an angle $\theta^{e}$ and a phase $\delta^{e}$, while the matrix $U_{v}$ depends on an angle $\theta^{v}$. In these two scenarios the leptonic $\mathrm{CPV}$ phases possess non-trivial values.

\section{Outlook}

The results obtained in Refs. [12-14,22, 25,30,33,35,37,39$42,136]$ and in many other studies (quoted in the present and the cited articles) show that a sufficiently precise measurement of the Dirac phase $\delta$ of the PMNS neutrino mixing matrix in the current and future neutrino oscillation experiments, combined with planned improvements of the precision on the neutrino mixing angles, can provide unique information about the possible discrete symmetry origin of the observed pattern of neutrino mixing and, correspondingly, about the existence of new fundamental symmetry in the lepton sector. Thus, these experiments will not simply provide a high precision data on the neutrino mixing and Dirac CPV parameters, but will probe at fundamental level the origin of the observed form of neutrino mixing. These future data will show, in particular, whether Nature followed the discrete symmetry approach for fixing the values of the three neutrino mixing angles and of the Dirac and Majorana $\mathrm{CP}$ violation phases of the PMNS neutrino mixing matrix. We are looking forward to these data and to the future exciting developments in neutrino physics.
Acknowledgements I would like to thank my former Ph.D. students I. Girardi and A.V. Titov for the fruitful and enjoyable collaboration and the numerous illuminating discussions of the problems considered in the present article. This work was supported in part by the INFN program on Theoretical Astroparticle Physics (TASP), by the European Union Horizon 2020 research and innovation programme under the Marie Sklodowska-Curie Grants 674896 and 690575, and by the World Premier International Research Center Initiative (WPI Initiative, MEXT), Japan.

Open Access This article is distributed under the terms of the Creative Commons Attribution 4.0 International License (http://creativecomm ons.org/licenses/by/4.0/), which permits unrestricted use, distribution, and reproduction in any medium, provided you give appropriate credit to the original author(s) and the source, provide a link to the Creative Commons license, and indicate if changes were made.

Funded by SCOAP ${ }^{3}$.

\section{References}

1. K. Nakamura, S.T. Petcov, in C. Patrignani, et al., [Particle Data Group]. Chin. Phys. C 40, 100001 (2016)

2. B. Pontecorvo, Zh Eksp, Teor. Fiz. 33, 549 (1957)

3. B. Pontecorvo, Zh Eksp, Teor. Fiz. 34, 247 (1958)

4. Z. Maki, M. Nakagawa, S. Sakata, Prog. Theor. Phys. 28, 870 (1962)

5. B. Pontecorvo, Zh Eksp, Teor. Fiz. 53, 1717 (1967)

6. A. de Gouvea, H. Murayama, Phys. Lett. B 747, 479 (2015)

7. A. de Gouvea, H. Murayama, Phys. Lett. B 573, 94 (2003)

8. L. Hall, H. Murayama, N. Weiner, Phys. Rev. Lett. 84, 2572 (2000)

9. G. Altarelli, F. Feruglio, Rev. Mod. Phys. 82, 2701 (2010)

10. H. Ishimori, Prog. Theor. Phys. Suppl. 183, 1 (2010). arXiv:1003.3552 [hep-th]

11. M. Tanimoto, A.I.P. Conf, Proc. 1666, 120002 (2015)

12. S.T. Petcov, Nucl. Phys. B 892, 400 (2015)

13. I. Girardi, S.T. Petcov, A.V. Titov, Eur. Phys. J. C 75, 345 (2015)

14. I. Girardi et al., Nucl. Phys. B 902, 1 (2016)

15. S.F. Ge, D.A. Dicus, W.W. Repko, Phys. Rev. Lett. 108, 041801 (2012)

16. S.F. Ge, D.A. Dicus, W.W. Repko, Phys. Lett. B 702, 220 (2011)

17. Y. Shimizu, M. Tanimoto, A. Watanabe, Prog. Theor. Phys. 126, 81 (2011). arXiv:1105.2929 [hep-ph]

18. Y. Shimizu, M. Tanimoto, K. Yamamoto, Mod. Phys. Lett. A 30, 1550002 (2015). arXiv:1405.1521 [hep-ph]

19. S.K. Kang, M. Tanimoto, Phys. Rev. D 91, 073010 (2015)

20. Y. Shimizu, M. Tanimoto, JHEP 1512, 132 (2015)

21. G.C. Branco, L. Lavoura, M.N. Rebelo, Phys. Lett. B 180, 264 (1986)

22. F. Feruglio, C. Hagedorn, R. Ziegler, JHEP 1307, 027 (2013)

23. M. Holthausen, M. Lindner, M.A. Schmidt, JHEP 1304, 122 (2013)

24. G.J. Ding et al., JHEP 1305, 084 (2013)

25. I. Girardi et al., JHEP 1402, 050 (2014)

26. G.J. Ding, S.F. King, A.J. Stuart, JHEP 1312, 006 (2013)

27. C. Hagedorn, A. Meroni, E. Molinaro, Nucl. Phys. B 891, 499 (2015)

28. C.C. Li, G.J. Ding, JHEP 1505, 100 (2015)

29. A. Di Iura, C. Hagedorn, D. Meloni, JHEP 1508, 037 (2015)

30. P. Ballett, S. Pascoli, J. Turner, Phys. Rev. D 92, 093008 (2015)

31. C.Y. Yao, G.J. Ding, Phys. Rev. D 94, 073006 (2016)

32. J. Turner, Phys. Rev. D 92, 116007 (2015)

33. I. Girardi, S.T. Petcov, A.V. Titov, Nucl. Phys. B 911, 754 (2016)

34. J.N. Lu, G.J. Ding, Phys. Rev. D 95, 015012 (2017)

35. J.T. Penedo, S.T. Petcov, A.V. Titov, JHEP 1712, 022 (2017) 
36. C.C. Li, J.N. Lu, G.J. Ding, JHEP 1802, 038 (2018)

37. A.D. Hanlon, S.F. Ge, W.W. Repko, Phys. Lett. B 729, 185 (2014)

38. P. Ballett et al., Phys. Rev. D 89, 016016 (2014)

39. I. Girardi, S.T. Petcov, A.V. Titov, Nucl. Phys. B 894, 733 (2015)

40. I. Girardi, S.T. Petcov, A.V. Titov, Int. J. Mod. Phys. A 30, 1530035 (2015). arXiv:1504.02402 [hep-ph]

41. S.K. Agarwalla et al., Eur. Phys. J. C 78, 286 (2018)

42. S.T. Petcov, A.V. Titov, Phys. Rev. D 97, 115045 (2018)

43. S.F. King, C. Luhn, Rept. Prog. Phys. 76, 056201 (2013)

44. D. Meloni, Front. in Phys. 5, 43 (2017). arXiv:1709.02662 [hep$\mathrm{ph}$ ]

45. S. Pakvasa, H. Sugawara, Phys. Lett. B 73, 61 (1978)

46. T. Brown et al., Phys. Rev. D 36, 256 (1984)

47. J. Kubo et al., Prog. Theor. Phys. 109, 795 (2003). [hep$\mathrm{ph} / 0302196 \mathrm{v} 4]$

48. C. Giunti, Nucl. Phys. B 908, 336 (2016)

49. S. Gariazzo et al. arXiv: 1703.00860

50. M. Maltoni, talk at the XXVIII Int. Conf. on Neutrino Physics and Astrophysics, June 5-9, 2018, Heidelberg, Germany

51. S.M. Bilenky, J. Hosek, S.T. Petcov, Phys. Lett. B 94, 495 (1980)

52. S.M. Bilenky, S.T. Petcov, Rev. Mod. Phys. 59, 671 (1987)

53. S.T. Petcov, Adv. High Energy Phys. 2013, 852987 (2013). arXiv:1303.5819 [hep-ph]

54. F. Capozzi et al., Phys. Rev. D 95, 096014 (2017)

55. I. Esteban et al., JHEP 1701, 087 (2017)

56. F. Capozzi et al., Phys. Rev. D 89, 093018 (2014)

57. I. Esteban et al. NuFIT webpage http://www.nu-fit.org (see v3.2: Three-neutrino fit based on data available in January 2018)

58. F. Capozzi, Prog. Part. Nucl. Phys. 102, 48 (2018). arXiv:1804.09678 [hep-ph]

59. N. Cabibbo, Phys. Lett. B 72, 333 (1978)

60. V.D. Barger, K. Whisnant, R.J.N. Phillips, Phys. Rev. Lett. 45, 2084 (1980)

61. P.I. Krastev, S.T. Petcov, Phys. Lett. B 205, 84 (1988)

62. C. Jarlskog, Z. Phys. C 29, 491 (1985)

63. P. Langacker et al., Nucl. Phys. B 282, 589 (1987)

64. S.M. Bilenky, S. Pascoli, S.T. Petcov, Phys. Rev. D 64, 053010 (2001)

65. S. Pascoli, S.T. Petcov, A. Riotto, Nucl. Phys. B 774, 1 (2007)

66. S. Pascoli, S.T. Petcov, A. Riotto, Phys. Rev. D 75, 083511 (2007)

67. C. Hagedorn, E. Molinaro, S.T. Petcov, JHEP 09, 115 (2009)

68. C. Hagedorn, E. Molinaro, Nucl. Phys. B 919, 404 (2017)

69. P. Chen, G.-J. Ding, S.F. King, JHEP 1603, 206 (2016)

70. Y. Shimizu, K. Takagi, M. Tanimoto, arXiv:1711.03863 [hep-ph]

71. C. Hagedorn et al., arXiv:1711.02866 [hep-ph]

72. A. Abada et al., JCAP 0604, 004 (2006)

73. E. Nardi et al., JHEP 0601, 164 (2006)

74. X. Qian, P. Vogel, Prog. Part. Nucl. Phys. 83, 1 (2015)

75. S.T. Petcov, M. Piai, Phys. Lett. B 533(94), 94 (2002)

76. S. Choubey, S.T. Petcov, M. Piai, Phys. Rev. D 68, 113006 (2003)

77. S.M. Bilenky, F. Capozzi, S.T. Petcov, Phys. Lett. B 772, 179 (2017)

78. J. Learned et al., Phys. Rev. D 78, 071302 (2008)

79. L. Zhan et al., Phys. Rev. D 78, 111103 (2008)

80. L. Zhan et al., Phys. Rev. D 79, 073007 (2009)

81. P. Ghoshal, S.T. Petcov, JHEP 1103, 058 (2011)

82. F. Capozzi, E. Lisi, A. Marrone, Phys. Rev. D 92, 093011 (2015)

83. S. Pascoli, S.T. Petcov, Phys. Lett. B 544, 239 (2002)

84. S.T. Petcov, S. Palomares-Ruiz, Nucl. Phys. B 712, 392 (2005)

85. S.T. Petcov, T. Schwetz, Nucl. Phys. B 740, 1 (2006)

86. F. An et al. [JUNO Collab.], J. Phys. G 43 (2016) 030401. arXiv: 1507.05613

87. S. Adrian-Martinez et al. [KM3Net Collab.], arXiv:1601.07459

88. M. G. Aartsen et al. [IceCube-PINGU Collab.], arXiv:1401.2046

89. R. Acciarri et al. [DUNE Collab.], arXiv:1512.06148, arXiv:1601.05471 and arXiv:1601.02984
90. S. Ahmed et al. [INO Collab.], arXiv: 1505.07380

91. F. Perrin, Comptes Rendus 197, 868 (1933)

92. E. Fermi, Nuovo Cim. 11, 1 (1934)

93. Ch. Kraus et al., Eur. Phys. J. C 40, 447 (2005)

94. V. Lobashev et al., Nucl. Phys. A 719, 153c (2003)

95. V.N. Aseev et al., Phys. Rev. D 84, 112003 (2011)

96. K. Eitel et al., Nucl. Phys. B (Proc. Suppl.) 143, 197 (2005)

97. K.N. Abazajian, M. Kaplinghat, Ann. Rev. Nucl. Part. Sci. 66, 401 (2016)

98. P.A.R. Ade et al., [Planck Collab.], Astron. Astrophys. 596, A13 (2016)

99. N. Aghanim et al. [Planck Collab.], Astrom. Astrophys. 596, A107 (2016)

100. K. Abe et al. [T2K Collab.], PTEP 2015 (2015) 043 C01. arXiv:1409.7469 [hep-ex]; arXiv:1609.04111 [hep-ex]

101. K. Abe et al. [Hyper-Kamiokande Proto-Collab.], PTEP 2015 (2015) 053C02. arXiv:1502.05199 [hep-ex]

102. K. Abe et al. [Hyper-Kamiokande Proto-Collab.], PTEP 2018 (2018) 063C01. arXiv:1611.06118v3 [hep-ex]

103. E. Ma, G. Rajasekaran, Phys. Rev. D 64, 113012 (2001)

104. E. Ma, Phys. Rev. D 70, 031901 (2004)

105. C.S. Lam, Phys. Lett. B 656, 193 (2007)

106. C.S. Lam, Phys. Rev. Lett. 101, 121602 (2008)

107. C.S. Lam, Phys. Rev. D 78, 073015 (2008)

108. P.H. Frampton, T.W. Kephart, Int. J. Mod. Phys. A 10, 4689 (1995). [hep-ph/9409330]

109. P.H. Frampton, T.W. Kephart, JHEP 0709, 110 (2007)

110. P.H. Frampton, T.W. Kephart, S. Matsuzaki, Phys. Rev. D 78, 073004 (2008)

111. A. Aranda, C.D. Carone, R.F. Lebed, Phys. Lett. B 474, 170 (2000)

112. A. Aranda, C.D. Carone, P. Meade, Phys. Rev. D 65, 013011 (2002)

113. L.L. Everett, A.J. Stuart, Phys. Rev. D 79, 085005 (2009)

114. F. Feruglio, A. Paris, JHEP 1103, 101 (2011)

115. A. Adulpravitchai, A. Blum, W. Rodejohann, New J. Phys. 11, 063026 (2009)

116. C.H. Albright, A. Dueck, W. Rodejohann, Eur. Phys. J. C 70, 1099 (2010)

117. J.E. Kim, M.S. Seo, JHEP 1102, 097 (2011)

118. E. Ma, Mod. Phys. Lett. A 21, 1917 (2006). arXiv:hep-ph/0607056

119. E. Ma, Phys. Lett. B 723, 161 (2013)

120. J.A. Escobar, C. Luhn, J. Math. Phys. 50, 013524 (2009)

121. R. de Adelhart Toorop, F. Feruglio, C. Hagedorn, Phys. Lett. B 703, 447 (2011)

122. R. de Adelhart Toorop, F. Feruglio, C. Hagedorn, Nucl. Phys. B 858, 437 (2012)

123. G.J. Ding, Nucl. Phys. B 862, 1 (2012)

124. S.F. King, C. Luhn, A.J. Stuart, Nucl. Phys. B 867, 203 (2013)

125. A. Meroni, S.T. Petcov, M. Spinrath, Phys. Rev. D 86, 113003 (2012)

126. C. Hagedorn, D. Meloni, Nucl. Phys. B 862, 691 (2012)

127. S. Antusch et al., Nucl. Phys. B 877, 772 (2013)

128. M.C. Chen et al., JHEP 1310, 112 (2013)

129. C.Y. Yao, G.J. Ding, Phys. Rev. D 92, 096010 (2015)

130. J. Gehrlein et al., Nucl. Phys. B 890, 539 (2014)

131. P.H. Frampton, S.T. Petcov, W. Rodejohann, Nucl. Phys. B 687, $31(2004)$

132. D. Marzocca et al., JHEP 1111, 009 (2011)

133. D. Marzocca et al., JHEP 1305, 073 (2013)

134. Y. Shimizu, M. Tanimoto, K. Yamamoto, Mod. Phys. Lett. A 30, 1550002 (2015). arXiv:1405.1521 [hep-ph]

135. S. T. Petcov, Sov. J. Nucl. Phys. 25 (1977) 340 [Yad. Fiz. 25 (1977) 641]; Erratum: Sov. J. Nucl. Phys. 25 (1977) 698 [Erratum: Yad. Fiz. 25 (1977) 1336]

136. G. Altarelli, F. Feruglio, L. Merlo, JHEP 0905, 020 (2009) 
137. M. Sanchez et al. [NOvA Collab.], talk at the XXVIII Int. Conf. on Neutrino Physics and Astrophysics, June 5-9, 2018, Heidelberg, Germany

138. S.F. King, C. Luhn, Nucl. Phys. B 820, 269 (2009)

139. C.S. Lam, Phys. Rev. Lett. 101, 121602 (2008)

140. P.F. Harrison, D.H. Perkins, W.G. Scott, Phys. Lett. B 530, 167 (2002)

141. Z.Z. Xing, Phys. Lett. B 533, 85 (2002)

142. L. Wolfenstein, Phys. Rev. D 18, 958 (1978)

143. G. Altarelli, F. Feruglio, Nucl. Phys. B 741, 215 (2006)

144. W. Grimus, L. Lavoura, JHEP 0809, 106 (2008)

145. C.H. Albright, W. Rodejohann, Eur. Phys. J. C 62, 599 (2009). arXiv:0812.0436

146. G. Altarelli, F. Feruglio, L. Merlo, Fortsch. Phys. 61, 507 (2013)

147. S.F. King, C. Luhn, JHEP 1109, 042 (2011)

148. S.F. King, JHEP 1307, 137 (2013)

149. S.F. King, C. Luhn, JHEP 1203, 036 (2012)

150. S.T. Petcov, Phys. Lett. B 110, 245 (1982)

151. F. Vissani, arXiv:hep-ph/9708483

152. V.D. Barger et al., Phys. Lett. B 437, 107 (1998)
153. A. Datta, F.S. Ling, P. Ramond, Nucl. Phys. B 671, 383 (2003)

154. Y. Kajiyama, M. Raidal, A. Strumia, Phys. Rev. D 76, 117301 (2007)

155. W. Rodejohann, Phys. Lett. B 671, 267 (2009)

156. F. Feruglio et al., Nucl. Phys. B 775, 120 (2007)

157. J. Gehrlein et al., JHEP 1611, 146 (2016)

158. J. Zhang, S. Zhou, JHEP 1608, 024 (2016)

159. C. Zhang et al. [Daya Bay Collab.], arXiv:1501.04991

160. J. Ling [Daya Bay Collab.], PoSICHEP2016 (2016) 467

161. E. Baussan et al., [ESSnuSB Collab.] Nucl. Phys. B 885, 127 (2014)

162. G.-J. Ding, L.L. Everett, A.J. Stuart, Nucl. Phys. B 857, 219 (2012)

163. S.T. Petcov, C.N. Leung, Phys. Lett. B 125, 461 (1983)

164. S.T. Petcov, S. Toshev, Phys. Lett. B 143, 175 (1984)

165. L.L. Everett, T. Garon, A.J. Stuart, JHEP 1504, 069 (2015)

166. F. Feruglio, C. Hagedorn, R. Ziegler, Eur. Phys. J. C 74, 2753 (2014)

167. G.J. Ding, S.F. King, Phys. Rev. D 89, 093020 (2014)

168. G.J. Ding, S.F. King, T. Neder, JHEP 1412, 007 (2014)

169. G.J. Ding, S.F. King, Phys. Rev. D 93, 025013 (2016) 